\title{
Redox-Neutral Borylation of Aryl Sulfonium Salts via C- S Activation Enabled by Light
}

Chen Huang, Jie Feng, Rui Ma, Shuaishuai Fang, Tao Lu, Weifang Tang,* Ding Du,* Jian Gao*

Department of Chemistry, China Pharmaceutical University, 24 Tong Jia Xiang, Nanjing, 210009

P. R. China

\section{Supporting Information}

Table of Contents

I. Materials and Methods.......................................................................S2

II. Details for the optimization of reaction conditions (Table S1) ................................S2

III. Experimental Procedures.........................................................S4

A. General procedure for the synthesis of aryl sulfonium triflates......................... S4

B. General procedure for the borylation of aryl sulfonium salts ................................S5

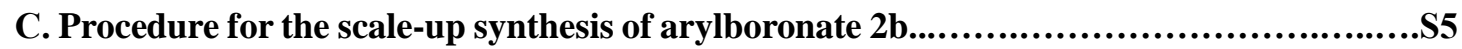

D. Procedures for mechanism study and control experiments...............................S5

IV. Characterization data...............................................................S8

V. References.............................................................................S21

VI. ${ }^{1} \mathrm{H}$ NMR, ${ }^{13} \mathrm{C}$ NMR and ${ }^{19}$ F NMR Spectra...............................................S23 


\section{Materials and Methods}

All reagents and solvents were commercially available (Aldrich, Aladdin, Alfa Aesar and Fluorochem) and were used without further purification. Flash column chromatography was performed over silica gel 200-300 mesh. Melting points were measured with a Fisher Johns apparatus and are uncorrected. ${ }^{1} \mathrm{H}$ NMR spectra were recorded on Bruker spectrometers at $300 \mathrm{MHz}$ or $400 \mathrm{MHz}$ and are reported relative to residual solvent signals. Data for ${ }^{1} \mathrm{H}$ NMR spectra are reported as follows: chemical shift $(\delta \mathrm{ppm})$, multiplicity, coupling constant $(\mathrm{Hz})$, integration. Data for ${ }^{13} \mathrm{C}$ NMR are reported in terms of chemical shift at $75 \mathrm{MHz}$ and reported in terms of chemical shift ( $\delta \mathrm{ppm})$. Data for ${ }^{19} \mathrm{~F}$ NMR are reported in terms of chemical shift at $282 \mathrm{MHz}$ and reported in terms of chemical shift $(\delta \mathrm{ppm})$. High-resolution mass spectra were obtained on Agilent 6520 Accurate-Mass Q-TOF LC/MS mass spectrometer with ESI source. UV-Vis absorption spectrum of sulfonium on Shimadzu UV 1800. The titled borylation reaction was conducted with a PL-DY1600 photo-reactor (Beijing Precise Technology Co., Ltd.).

\section{Details for the optimization of reaction conditions (Table S1)}

Table S1. Optimization of Reaction Conditions ${ }^{\mathrm{a}}$

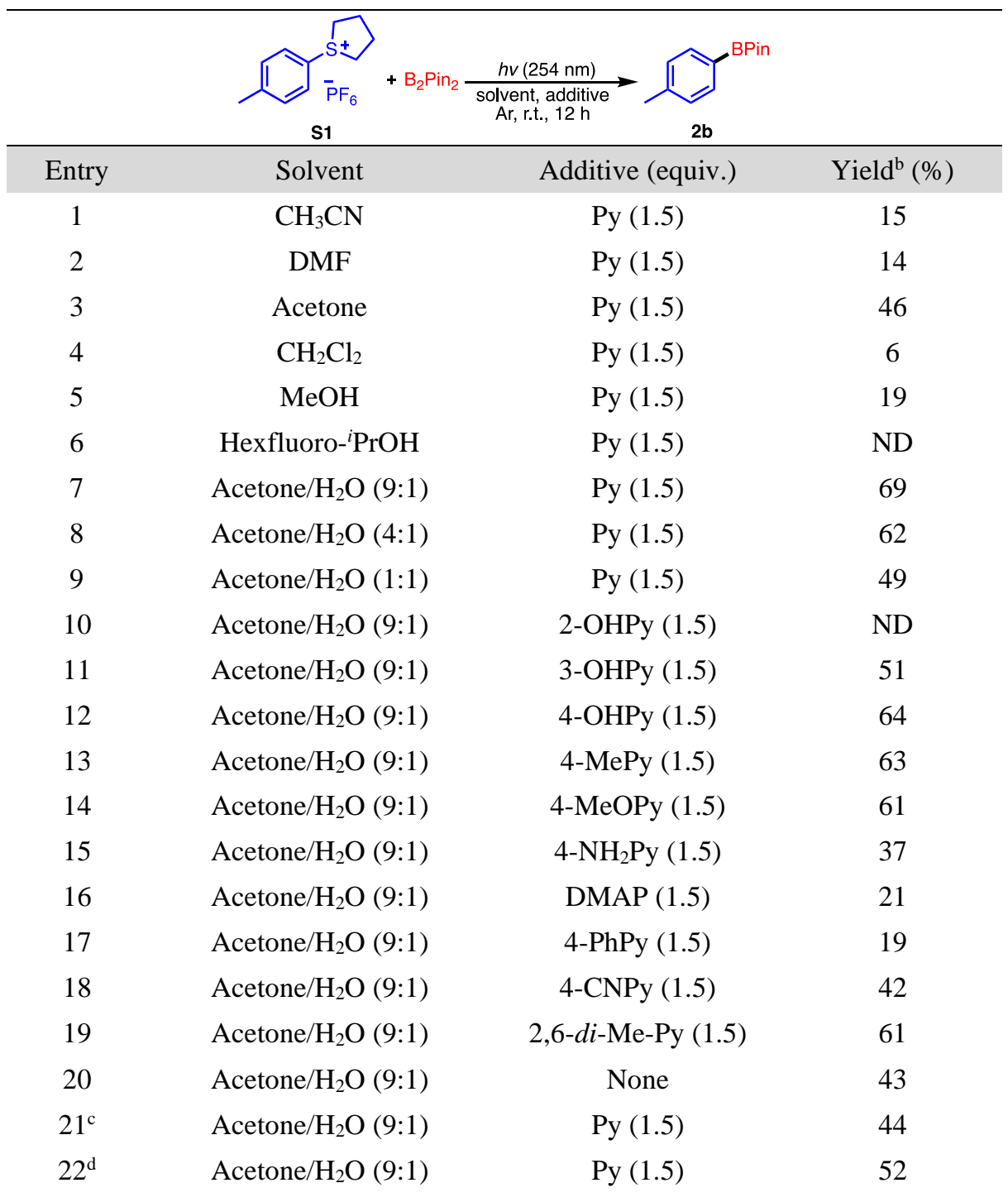




\begin{tabular}{|c|c|c|c|}
\hline $23^{\mathrm{e}}$ & Acetone $/ \mathrm{H}_{2} \mathrm{O}(9: 1)$ & Py (1.5) & 62 \\
\hline $24^{\mathrm{f}}$ & Acetone $/ \mathrm{H}_{2} \mathrm{O}(9: 1)$ & Py (1.5) & 68 \\
\hline 25 & Acetone $/ \mathrm{H}_{2} \mathrm{O}(9: 1)$ & Py $(2.0)$ & 68 \\
\hline 26 & Acetone $/ \mathrm{H}_{2} \mathrm{O}(9: 1)$ & Py (3.0) & 71 \\
\hline 27 & Acetone $/ \mathrm{H}_{2} \mathrm{O}(9: 1)$ & Py (4.0) & 77 \\
\hline 28 & Acetone/ $\mathrm{H}_{2} \mathrm{O}(9: 1)$ & Py (5.0) & $82(81)^{\mathrm{g}}$ \\
\hline $29^{\mathrm{h}}$ & Acetone $/ \mathrm{H}_{2} \mathrm{O}(9: 1)$ & Py (5.0) & ND \\
\hline $30^{i}$ & Acetone/ $\mathrm{H}_{2} \mathrm{O}(9: 1)$ & Py (5.0) & $81^{\mathrm{g}}$ \\
\hline $31^{\mathrm{j}}$ & Acetone $/ \mathrm{H}_{2} \mathrm{O}(9: 1)$ & Py (5.0) & $69^{g}$ \\
\hline $32^{\mathrm{k}}$ & Acetone/ $\mathrm{H}_{2} \mathrm{O}(9: 1)$ & Py (5.0) & $65^{\mathrm{g}}$ \\
\hline \multicolumn{4}{|c|}{$\begin{array}{l}{ }^{a} \text { Reaction conditions: } \mathbf{S} 1(0.1 \mathrm{mmol}) ; \mathrm{B}_{2} \operatorname{Pin}_{2}(3.5 \text { equiv. }) \text {; solvent, } 1.0 \mathrm{~mL} ; h v(254 \\
\text { nm); Ar; r.t.; } 12 \text { h. }{ }^{b} \mathrm{NMR} \text { yield with mesitylene as internal standard. }{ }^{c} \mathrm{~B}_{2} \operatorname{Pin}_{2}(1.5 \\
\text { equiv. }) .{ }^{d} \mathrm{~B}_{2} \mathrm{Pin}_{2}(2.0 \text { equiv. }) .{ }^{e} \mathrm{~B}_{2} \mathrm{Pin}_{2}(3.0 \text { equiv. }) .{ }^{f} \mathrm{~B}_{2} \mathrm{Pin}_{2}(4.0 \text { equiv. }) .{ }^{g} \text { Isolated } \\
\text { yield. }{ }^{h} \text { The reaction was conducted in dark. }{ }^{i} \mathbf{1 b} \text { instead of } \mathbf{S 1} .{ }^{j} \mathbf{S 2} \text { instead of } \mathbf{S 1} .{ }^{k} \\
\mathbf{S 3} \text { instead of S1. Py: pyridine; DMAP: } 4-(N, N \text {-dimethyl)-aminopyridine; ND: not } \\
\text { detected. }\end{array}$} \\
\hline
\end{tabular}

The borylation of sulfonium S1 under UV-irradiation (254 nm) using $\mathrm{B}_{2} \mathrm{Pin}_{2}$ (bis(pinacolato)diboron) as borylation reagent was chosen as the model reaction for the optimization of reaction conditions. Firstly, a serious of solvents were examined in the presence of pyridine, which acted as an activating additive toward $\mathrm{B}_{2} \mathrm{Pin}_{2}$ (Table S1). To our delight, the desired arylboronate ester $\mathbf{2 b}$ could be detected in most of the common polar solvents (Table S1, entries 1-6) and 46\% NMR yield could be achieved when the borylation was conducted in acetone (Table S1, entry 3 ). Considering that the hydrophilicity of the sulfonium salts, water was added as a cosolvent in order to increase the solubility of $\mathbf{S 1}$. As a result, $69 \%$ yield for $\mathbf{2 b}$ was obtained with ten percent of water added in acetone (Table S1, entry 7). Nevertheless, a negative effect for the borylation was found when more water was added, which might ascribe to the sensibility of boronate ester to water (Table S1, entries 8 and 9). Aiming to further facilitate this borylation reaction, we then turned our attention to the additive and various of substituted pyridines were investigated in acetone/water ( $/ \mathrm{v}=9: 1)$. Resultly, it was observed that all the substituted pyridines that bearing either electron-donating groups or electron-withdrawing groups displayed worse activity toward this borylation compared with pyridine (Table S1, entries 10-19). Additionally, there was an obvious decrease on the yield of 2b when no additive was used (Table S1, entry 20). Therefore, pyridine could be chosen as the optimal additive. Next, the amount of $\mathrm{B}_{2} \mathrm{Pin}_{2}$ was studied and it was found that up to 3.5 equivalent of $\mathrm{B}_{2} \mathrm{Pin}_{2}$ was necessary for a satisfactory yield of the desired product (Table S1, entries 21-24). Given that the activating role of pyridine toward $\mathrm{B}_{2} \mathrm{Pin}_{2}$, it could be speculated that the amount of pyridine might also be influential to this transformation. Fortunately, $82 \%$ NMR yield for $\mathbf{2 b}$ could be obtained accompanied with $81 \%$ isolated yield in the presence of 5.0 equivalent of pyridine (Table 
S1, entries 25-28). Last, it was proved that the borylation of sulfonium could not proceed in dark at all, which could convince the indispensable role of photoirradiation to the sulfonium in the absence of any other catalysts (Table S1, entry 29). Given the ease of the modification for aryl sulfonium salts, several different sulfonium salts were synthesized and their activity toward borylation was also evaluated. To our delight, all the sulfonium salts were compatible with our protocol under the optimal conditions (Table S1, entries 30-32). Notably, the relatively simple sulfonium triflate $\mathbf{1 b}$, which can be easily synthesized by simple methylation of corresponding methyl aryl sulfide, displayed the same activity just as sulfonium $\mathbf{S 1}$ under the identical reaction conditions.

\section{Experimental Procedures}

\section{A. General procedure for the synthesis of aryl sulfonium triflates.}

Sulfonium triflates 1a-1z were synthesized according to Scheme S1 using a modified variant of the method reported by Yorimitsu. ${ }^{1}$

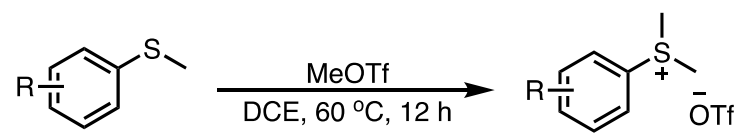

Scheme S1. Synthesis of Arylsufonium Triflates.

A flask was charged with aryl methyl thioether ( $5 \mathrm{mmol}, 1.0$ equiv.) and 1,2-dichloroethane (20 $\mathrm{mL})$. Methyltrifluoromethanesulfonate ( $6.5 \mathrm{mmol}, 1.3$ equiv.) was added and the resulting mixture was stirred for $12 \mathrm{~h}$ at $60^{\circ} \mathrm{C}$ in an oil-bath. After the completion of the reaction as indicated by TLC, the mixture was concentrated under a reduced pressure. The product was purified by recrystallization from $\mathrm{CH}_{2} \mathrm{Cl}_{2} / \mathrm{Et}_{2} \mathrm{O}$ to give pure colorless crystals.

Other sulfonium salts (Scheme S2) used in this study were synthesized according to the reported methods ${ }^{1-4}$ and their characterization data were consistent with the literatures.

(a)

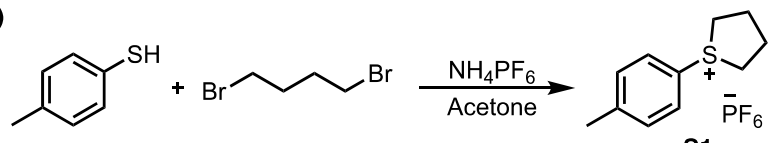

(b)

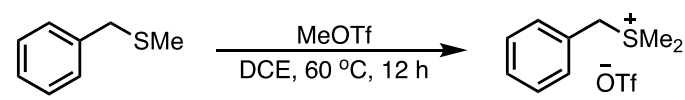

(c)

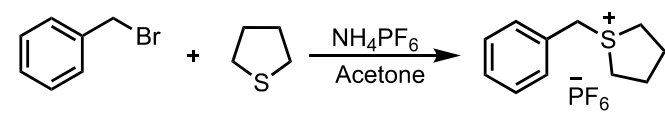

(d) BzS-2

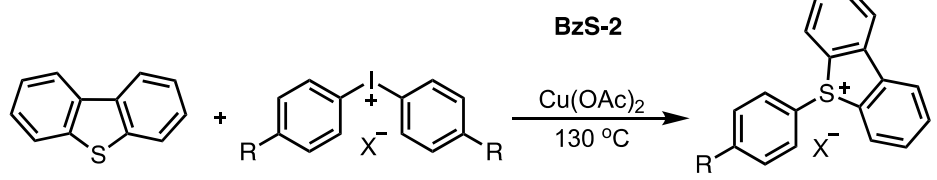

(e)

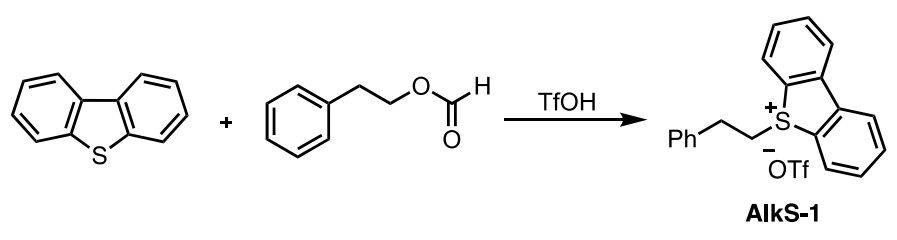

Scheme S2. Synthesis of Other Sulfonium Salts. 


\section{B. General procedure for the borylation of aryl sulfonium slats.}

The borylation of aryl sulfonium salts were conducted with a PL-DY1600 photoreactor with UV lamps (16*10W, 254 nm, from Beijing Precise Technology Co., Ltd.).

To a screw-capped vial with septum containing a magnetic stir bar was added the aryl sulfonium (0.1 mmol, 1.0 equiv.), $\mathrm{B}_{2} \mathrm{Pin}_{2}$ ( $0.35 \mathrm{mmol}, 3.5$ equiv.), pyridine ( $0.5 \mathrm{mmol}, 5.0$ equiv.), followed by acetone $(0.9 \mathrm{~mL})$ and $\mathrm{H}_{2} \mathrm{O}(0.1 \mathrm{~mL})$. The vial was closed and the mixture was degassed by freezing in liquid nitrogen and by purging with argon for 3 times. After that, parafilm was placed around the lid of the vial and the mixture was stirred under UV-irradiation $(254 \mathrm{~nm})$. After $12 \mathrm{~h}$, the reaction mixture was concentrated in vacuo. The crude residue was purified by silica gel flash column chromatography (petroleum ether : ethyl acetate $=100: 1$ ) to provide the pure borylated products.

All of the aryl boronate esters obtained were known compounds and the characterization data were in consistent with the reported literatures ${ }^{2-8}$.

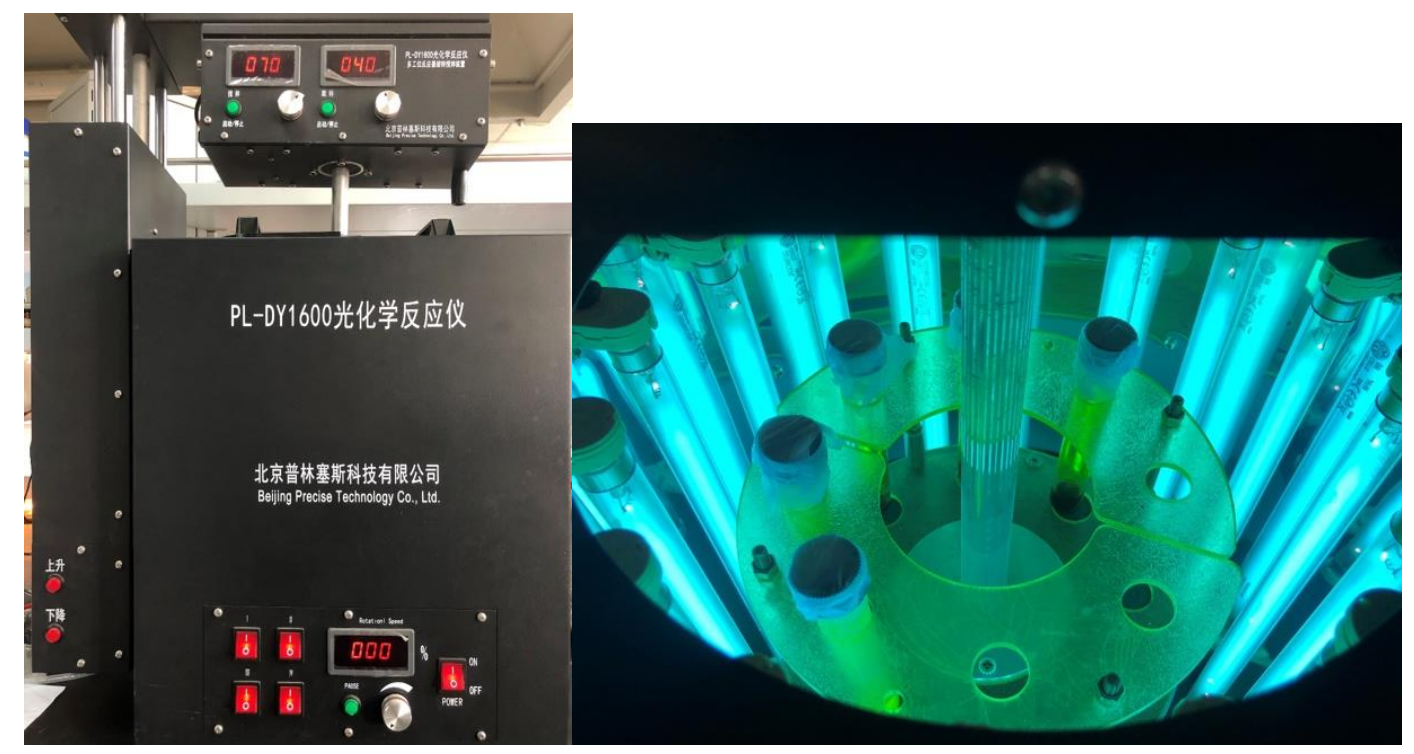

\section{Procedure for the scale-up synthesis of arylboronate $\mathbf{2 b}$.}

To a screw-capped vial with septum containing a magnetic stir bar was added the aryl sulfonium $\mathbf{1 b}$ ( 5 mmol, 1.0 equiv.), $\mathrm{B}_{2} \mathrm{Pin}_{2}$ ( $16.5 \mathrm{mmol}, 3.5$ equiv.), pyridine ( $25 \mathrm{mmol}, 5.0$ equiv.), followed by acetone $(22.5 \mathrm{~mL})$ and $\mathrm{H}_{2} \mathrm{O}(2.5 \mathrm{~mL})$. The vial was closed and the mixture was degassed by freezing in liquid nitrogen and by purging with argon for 3 times. After that, parafilm was placed around the lid of the vial and the mixture was stirred under UV-irradiation $(254 \mathrm{~nm})$. After $12 \mathrm{~h}$, the reaction mixture was concentrated in vacuo. The crude residue was purified by silica gel flash column chromatography (petroleum ether : ethyl acetate $=100: 1)$ to provide the pure products $\mathbf{2} \mathbf{b}(795 \mathrm{mg}$ $73 \%)$.

D. Procedures for mechanism study and control experiments.

(a) Radical trapping experiment: 
To a screw-capped vial with septum containing a magnetic stir bar was added the dimethyl $(p-$ tolyl)sulfonium triflate $\mathbf{1 b}$ ( $0.1 \mathrm{mmol}, 1.0$ equiv.), $\mathrm{B}_{2} \operatorname{Pin}_{2}$ ( $0.35 \mathrm{mmol}, 3.5$ equiv.), pyridine ( 0.5 mmol, 5.0 equiv.), TEMPO (3.0 equiv.) or BHT (3.0 equiv.), followed by acetone $(0.9 \mathrm{~mL})$ and $\mathrm{H}_{2} \mathrm{O}(0.1 \mathrm{~mL})$. The vial was closed and the mixture was degassed by freezing in liquid nitrogen and by purging with argon for 3 times. After that, parafilm was placed around the lid of the vial and the mixture was stirred under UV-irradiation $(254 \mathrm{~nm})$. After $12 \mathrm{~h}$, the reaction mixture was concentrated in vacuo. The yield of the desired product was detected by NMR and the radical trapping products were detected by LC-MS.

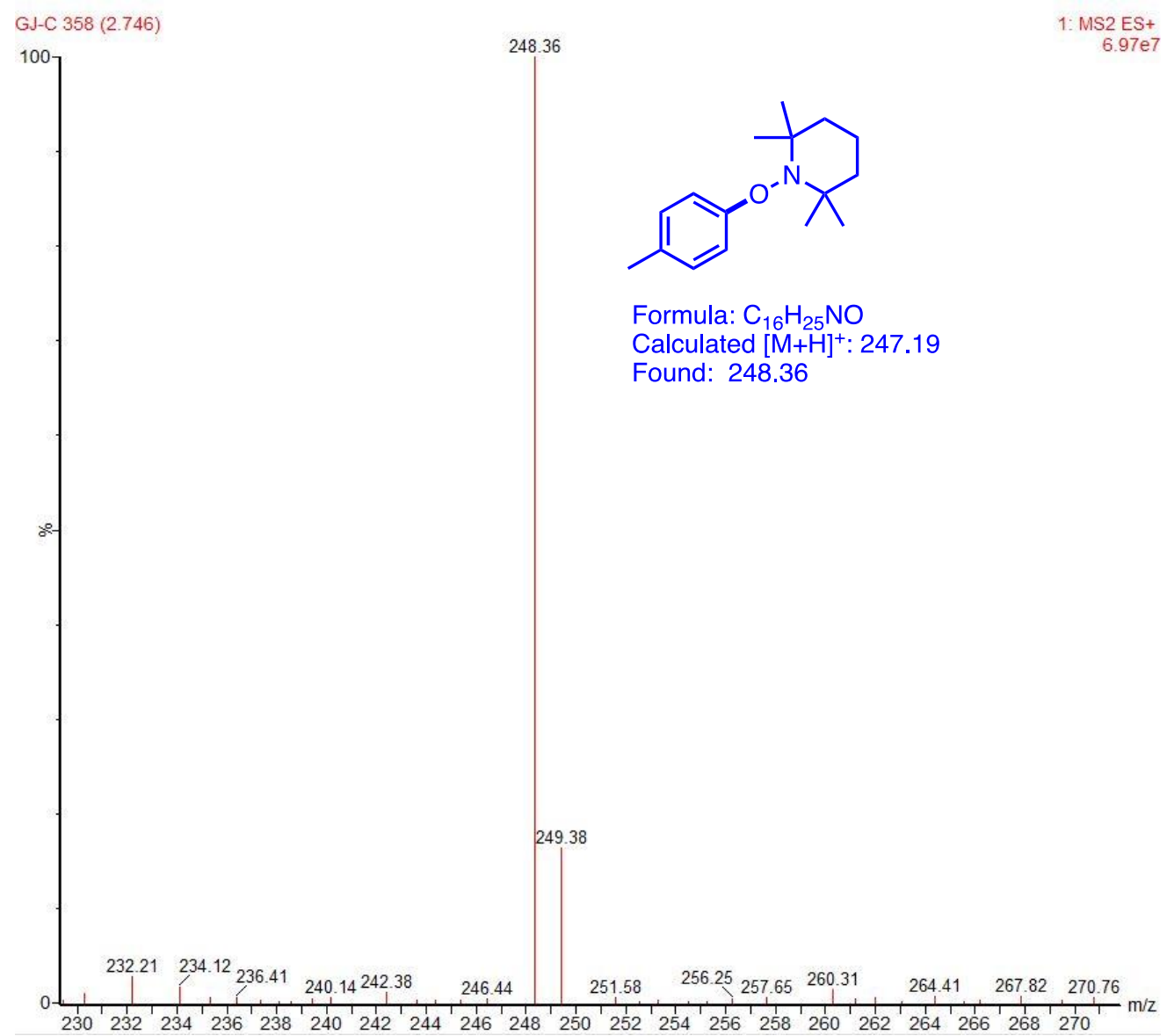




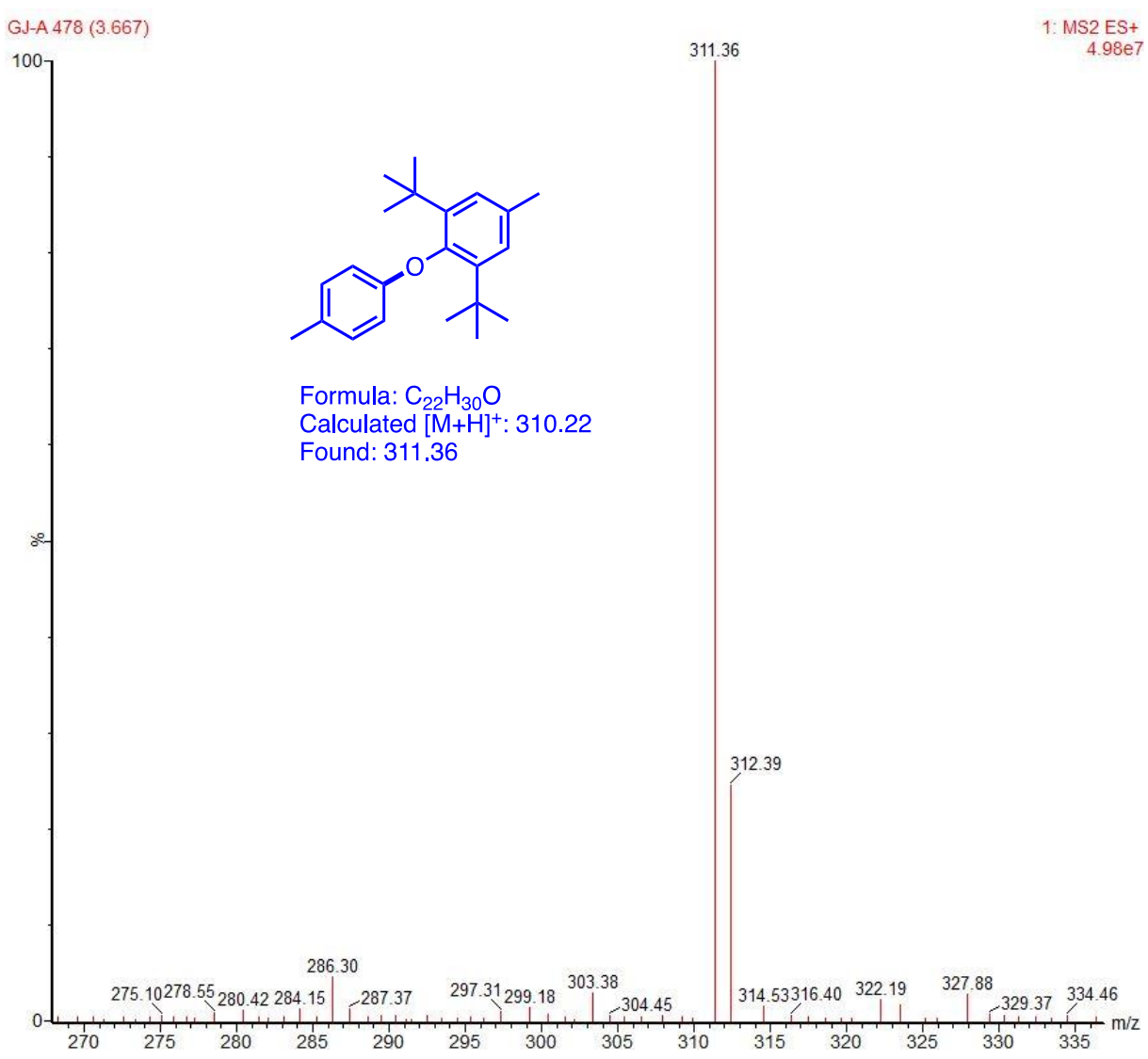

\section{(b) $S$-motif capturing experiment:}

To a screw-capped vial with septum containing a magnetic stir bar was added $S$-( $p$-tolyl) tetramethylenesulfonium hexafluorophosphate $\mathbf{S 1}\left(0.1 \mathrm{mmol}, 1.0\right.$ equiv.), $\mathrm{B}_{2} \mathrm{Pin}_{2}(0.35 \mathrm{mmol}, 3.5$ equiv.), pyridine ( $0.5 \mathrm{mmol}, 5.0$ equiv.), followed by acetone $(0.9 \mathrm{~mL})$ and $\mathrm{H}_{2} \mathrm{O}(0.1 \mathrm{~mL})$. The vial was closed and the mixture was degassed by freezing in liquid nitrogen and by purging with argon for 3 times. After that, parafilm was placed around the lid of the vial and the mixture was stirred under UV-irradiation $(254 \mathrm{~nm})$. After $12 \mathrm{~h}$, the reaction mixture was concentrated in vacuo. The $S$ motif, i.e., tetrahydrothiophene, was detected by NMR and its NMR yield was determined using mesitylene as internal standard.

\section{(c) Control experiment:}

To a screw-capped vial with septum containing a magnetic stir bar was added the dimethyl((4methoxyphenyl)sulfonium triflate 1 e $(0.1 \mathrm{mmol}, 1.0$ equiv. $), \mathrm{B}_{2} \mathrm{Pin}_{2}(0.35 \mathrm{mmol}, 3.5$ equiv.), pyridine $\left(0.5 \mathrm{mmol}, 5.0\right.$ equiv.), followed by acetone $(0.9 \mathrm{~mL})$ and $\mathrm{H}_{2} \mathrm{O}(0.1 \mathrm{~mL})$. The vial was closed and the mixture was degassed by freezing in liquid nitrogen and by purging with argon for 3 times. After that, parafilm was placed around the lid of the vial and the mixture was stirred under 
UV-irradiation (254 nm). After $12 \mathrm{~h}$, the reaction mixture was concentrated in vacuo. The desired product 4 was isolated by silica gel flash column chromatography (petroleum ether : ethyl acetate $=$ 10:1) and was confirmed by NMR.

\section{(d) Chemoselective evaluation:}

Intramolecular: To a screw-capped vial with septum containing a magnetic stir bar was added the 4-haloarylsulfoniums 1i-1k ( $0.1 \mathrm{mmol}, 1.0$ equiv.), $\mathrm{B}_{2} \mathrm{Pin}_{2}$ ( $0.35 \mathrm{mmol}, 3.5$ equiv.), pyridine ( 0.5 mmol, 5.0 equiv.), followed by acetone $(0.9 \mathrm{~mL})$ and $\mathrm{H}_{2} \mathrm{O}(0.1 \mathrm{~mL})$. The vial was closed and the mixture was degassed by freezing in liquid nitrogen and by purging with argon for 3 times. After that, parafilm was placed around the lid of the vial and the mixture was stirred under UV-irradiation $(254 \mathrm{~nm})$. After $12 \mathrm{~h}$, the reaction mixture was concentrated in vacuo and the crude residue was purified by silica gel flash column chromatography (petroleum ether : ethyl acetate $=100: 1$ ) to provide the pure products $\mathbf{2 i - 2 k}$.

Intermolecular: To a screw-capped vial with septum containing a magnetic stir bar was added dimethyl( $p$-tolyl)sulfonium triflate $\mathbf{1 b}(0.1 \mathrm{mmol}, 1.0$ equiv.), 4-Bromobenzotrifluoride $(0.1 \mathrm{mmol}$, 1.0 equiv.), $\mathrm{B}_{2} \mathrm{Pin}_{2}$ ( $0.35 \mathrm{mmol}, 3.5$ equiv.), pyridine ( $0.5 \mathrm{mmol}, 5.0$ equiv.), followed by acetone $(0.9 \mathrm{~mL})$ and $\mathrm{H}_{2} \mathrm{O}(0.1 \mathrm{~mL})$. The vial was closed and the mixture was degassed by freezing in liquid nitrogen and by purging with argon for 3 times. After that, parafilm was placed around the lid of the vial and the mixture was stirred under UV-irradiation ( $254 \mathrm{~nm}$ ). After $12 \mathrm{~h}$, the reaction mixture was concentrated in vacuo and was added mesitylene as internal standard to calculate the NMR yield.

\section{Characterization data.}<smiles>C[S+](C)c1ccccc1</smiles>

$1 a$

dimethyl(phenyl)sulfonium OTf (1a). White solid (1.2 g, 85\%); mp: 101-103 ${ }^{\circ} \mathrm{C}$; ${ }^{1} \mathrm{H}$ NMR (300 MHz, DMSO- $\left.d_{6}\right): \delta=8.08(\mathrm{~d}, J=1.1 \mathrm{~Hz}, 1 \mathrm{H}), 8.05(\mathrm{~d}, J=1.6 \mathrm{~Hz}, 1 \mathrm{H}), 7.82-7.78(\mathrm{~m}, 1 \mathrm{H}), 7.77-$ $7.74(\mathrm{~m}, 1 \mathrm{H}), 7.72-7.69(\mathrm{~m}, 1 \mathrm{H}), 3.27(\mathrm{~s}, 6 \mathrm{H}) . ;{ }^{13} \mathrm{C}$ NMR (75 MHz, DMSO- $\left.d_{6}\right): \delta=134.1,130.9$, 130.2, 127.2, 28.6; ESI-HRMS calcd for $\mathrm{C}_{8} \mathrm{H}_{11} \mathrm{~S}^{+}$[M-OTf] ${ }^{+}$: 139.0576, found 139.0590 .<smiles></smiles>

1b 
dimethyl(p-tolyl)sulfonium OTf (1b). Grey solid (1.3 g, 91\%); mp: 97-99 ${ }^{\circ} \mathrm{C} ;{ }^{1} \mathrm{H}$ NMR (400 MHz, DMSO- $\left.d_{6}\right): \delta=7.95(\mathrm{~d}, J=8.2 \mathrm{~Hz}, 2 \mathrm{H}), 7.54(\mathrm{~d}, J=8.2 \mathrm{~Hz}, 2 \mathrm{H}), 3.23(\mathrm{~s}, 6 \mathrm{H}), 2.42(\mathrm{~s}, 3 \mathrm{H}) ;{ }^{13} \mathrm{C}$ NMR (75 MHz, DMSO- $d_{6}$ ): $\delta=144.9,131.4,130.2,123.6,28.8,21.4$; ESI-HRMS calcd for $\mathrm{C}_{9} \mathrm{H}_{13} \mathrm{~S}^{+}$[M-OTf] $]^{+}: 153.0732$, found 153.0753 .<smiles>C[Sb](C)c1ccc(C(C)(C)C)cc1</smiles>

(4-(tert-butyl)phenyl)dimethylsulfonium OTf (1c). White solid (1.5 g, 91\%); mp: $94-95{ }^{\circ} \mathrm{C} ;{ }^{1} \mathrm{H}$ NMR (300 MHz, DMSO- $\left.d_{6}\right): \delta=7.98(\mathrm{~d}, J=8.6 \mathrm{~Hz}, 2 \mathrm{H}), 7.74(\mathrm{~d}, J=8.7 \mathrm{~Hz}, 2 \mathrm{H}), 3.24(\mathrm{~s}, 6 \mathrm{H})$, 1.32 (s, 9H); ${ }^{13} \mathrm{C}$ NMR (75 MHz, DMSO- $\left.d_{6}\right): \delta=157.5,130.2,127.9,123.8,35.5,31.2,28.7$; ESIHRMS calcd for $\mathrm{C}_{12} \mathrm{H}_{19} \mathrm{~S}^{+}[\mathrm{M}-\mathrm{OTf}]^{+}:$195.1202, found 195.1202.<smiles></smiles>

1d

(4-hydroxyphenyl)dimethylsulfonium OTf (1d). White solid (1.2 g, 82\%); mp: $55-57{ }^{\circ} \mathrm{C} ;{ }^{1} \mathrm{H}$ NMR (300 MHz, DMSO- $\left.d_{6}\right): \delta=10.76(\mathrm{~s}, 1 \mathrm{H}), 7.88(\mathrm{~d}, J=8.9 \mathrm{~Hz}, 2 \mathrm{H}), 7.04(\mathrm{~d}, J=8.9 \mathrm{~Hz}, 2 \mathrm{H})$, $3.18(\mathrm{~s}, 6 \mathrm{H}) ;{ }^{13} \mathrm{C}$ NMR $\left(101 \mathrm{MHz}, \mathrm{DMSO}-d_{6}\right): \delta=162.8,132.6,117.7,114.4,29.3$; ESI-HRMS calcd for $\mathrm{C}_{8} \mathrm{H}_{11} \mathrm{OS}^{+}$[M-OTf] $]^{+}$: 155.0525, found 155.0531 .

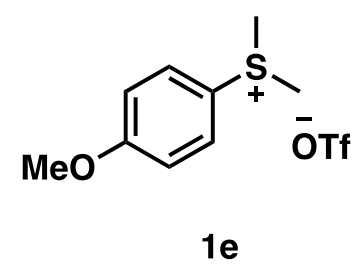

(4-methoxyphenyl)dimethylsulfonium OTf (1e). White solid (1.4 g, 92\%); mp: 77-79 ${ }^{\circ} \mathrm{C} ;{ }^{1} \mathrm{H}$ NMR (300 MHz, DMSO- $\left.d_{6}\right): \delta=8.01(\mathrm{~d}, J=9.0 \mathrm{~Hz}, 2 \mathrm{H}), 7.26(\mathrm{~d}, J=9.0 \mathrm{~Hz}, 2 \mathrm{H}), 3.87(\mathrm{~s}, 3 \mathrm{H})$, 3.21 (s, 6H); ${ }^{13} \mathrm{C}$ NMR (75 MHz, DMSO- $\left.d_{6}\right): \delta=163.9,132.5,116.8,116.8,56.4,29.2$; ESI-HRMS calcd for $\mathrm{C}_{9} \mathrm{H}_{13} \mathrm{OS}^{+}$[M-OTf] $]^{+}$: 169.0682, found 169.0685 .<smiles>C=CCOc1ccc([S+](C)=C(C)[O])cc1</smiles>

$1 f$

(4-(allyloxy)phenyl)dimethylsulfonium OTf (1f). White solid (1.5 g, 88\%); mp: $78-79{ }^{\circ} \mathrm{C} ;{ }^{1} \mathrm{H}$ NMR $\left(400 \mathrm{MHz}\right.$, DMSO- $\left.d_{6}\right) \delta=8.00(\mathrm{~d}, J=9.0 \mathrm{~Hz}, 2 \mathrm{H}), 7.27(\mathrm{~d}, J=9.1 \mathrm{~Hz}, 2 \mathrm{H}), 6.04$ (ddt, $J=$ $17.2,10.5,5.2 \mathrm{~Hz}, 1 \mathrm{H}), 5.45(\mathrm{dq}, J=17.3,1.6 \mathrm{~Hz}, 1 \mathrm{H}), 5.32(\mathrm{dq}, J=10.5,1.4 \mathrm{~Hz}, 1 \mathrm{H}), 4.71(\mathrm{dt}, J$ $=5.2,1.5 \mathrm{~Hz}, 2 \mathrm{H}), 3.22(\mathrm{~s}, 6 \mathrm{H}) ;{ }^{13} \mathrm{C}$ NMR $\left(101 \mathrm{MHz}, \mathrm{DMSO}-d_{6}\right): \delta=162.7,133.2,132.5,118.6$, 
117.1, 116.9, 69.2, 29.1; ESI-HRMS calcd for $\mathrm{C}_{11} \mathrm{H}_{15} \mathrm{OS}^{+}[\mathrm{M}-\mathrm{OTf}]^{+}$: 195.0838, found 195.0838.<smiles>C[As](C)c1ccc(C2OCC(C)(C)CO2)cc1</smiles>

$1 \mathrm{~g}$

(4-(5,5-dimethyl-1,3-dioxan-2-yl)phenyl)dimethylsulfonium OTf (1g). White solid (1.6 g, 82\%); mp:160-161 ${ }^{\circ} \mathrm{C} ;{ }^{1} \mathrm{H}$ NMR $\left(300 \mathrm{MHz}\right.$, DMSO- $\left.d_{6}\right) \delta=8.07(\mathrm{~d}, J=8.6 \mathrm{~Hz}, 2 \mathrm{H}), 7.74(\mathrm{~d}, J=8.4 \mathrm{~Hz}$, 2H), 5.55 (s, 1H), 3.80-3.60 (m, 4H), 3.26 (s, 6H), 1.18 (s, 3H), 0.77 (s, 3H); ${ }^{13} \mathrm{C}$ NMR (75 MHz, DMSO- $\left.d_{6}\right): \delta=144.3,130.3,128.5,127.5,99.8,77.0,30.4,28.7,23.1,21.8$; ESI-HRMS calcd for $\mathrm{C}_{14} \mathrm{H}_{21} \mathrm{O}_{2} \mathrm{~S}^{+}[\mathrm{M}-\mathrm{OTf}]^{+}:$253.1257, found 253.1252.<smiles></smiles>

$1 \mathrm{~h}$

dimethyl(4-(2,5,5-trimethyl-1,3-dioxan-2-yl)phenyl)sulfonium (1h). White solid (1.7 g, 82\%); mp: $156-158{ }^{\circ} \mathrm{C} ;{ }^{1} \mathrm{H}$ NMR $\left(400 \mathrm{MHz}, \mathrm{DMSO}-d_{6}\right) \delta=8.12(\mathrm{~d}, J=8.6 \mathrm{~Hz}, 2 \mathrm{H}), 7.66(\mathrm{~d}, J=8.6 \mathrm{~Hz}$, 2H), 3.47 (d, $J=11.3 \mathrm{~Hz}, 2 \mathrm{H}), 3.28$ (s, 6H), $3.22(\mathrm{~d}, J=11.1 \mathrm{~Hz}, 2 \mathrm{H}), 1.45$ (s, 3H), $1.16(\mathrm{~s}, 3 \mathrm{H})$, $0.58(\mathrm{~s}, 3 \mathrm{H}) ;{ }^{13} \mathrm{C}$ NMR $\left(75 \mathrm{MHz}, \mathrm{DMSO}-d_{6}\right): \delta=147.5,130.9,128.8,126.6,99.5,71.4,31.4,30.0$, 28.6, 22.8, 22.0; ESI-HRMS calcd for $\mathrm{C}_{15} \mathrm{H}_{23} \mathrm{O}_{2} \mathrm{~S}^{+}$[M-OTf] $]^{+}$: 267.1413, found 267.1412.<smiles>C[S+](C)c1ccc(F)cc1</smiles>

$1 \mathbf{i}$

(4-fluorophenyl)dimethylsulfonium OTf (1i). Grey solid (1.2 g, 84\%); mp: 84-85 ${ }^{\circ} \mathrm{C}$; ${ }^{1} \mathrm{H}$ NMR (300 MHz, DMSO- $\left.d_{6}\right) ; \delta=8.19-8.14(\mathrm{~m}, 2 \mathrm{H}), 7.65-7.59(\mathrm{~m}, 2 \mathrm{H}), 3.26(\mathrm{~s}, 6 \mathrm{H}) ;{ }^{13} \mathrm{C} \mathrm{NMR}(75 \mathrm{MHz}$, DMSO- $\left.d_{6}\right): \delta=165.4(\mathrm{~d}, J=247.5 \mathrm{~Hz}), 133.5(\mathrm{~d}, J=7.5 \mathrm{~Hz}), 122.8(\mathrm{~d}, J=3.7 \mathrm{~Hz}), 118.1(\mathrm{~d}, J=23.2$ $\mathrm{Hz}$ ), 28.9; ${ }^{19} \mathrm{~F}$ NMR (282 MHz, DMSO- $\left.d_{6}\right) \delta=-77.77,-104.87$; ESI-HRMS calcd for $\mathrm{C}_{8} \mathrm{H}_{10} \mathrm{FS}^{+}[\mathrm{M}-$ $\mathrm{OTf}^{+}:$: 157.0482, found 157.0479 .<smiles>C[S+](C)c1ccc(Cl)cc1</smiles>

$1 \mathrm{j}$

(4-chlorophenyl)dimethylsulfonium OTf (1j). White solid (1.3 g, 86\%); mp: $117-119{ }^{\circ} \mathrm{C} ;{ }^{1} \mathrm{H}$ NMR (300 MHz, DMSO- $\left.d_{6}\right): \delta=8.10(\mathrm{~d}, J=8.8 \mathrm{~Hz}, 2 \mathrm{H}), 7.84(\mathrm{~d}, J=8.7 \mathrm{~Hz}, 2 \mathrm{H}), 3.27(\mathrm{~s}, 6 \mathrm{H})$; 
${ }^{13} \mathrm{C}$ NMR (75 MHz, DMSO- $\left.d_{6}\right): \delta=139.2,132.3,130.9,126.2,28.7$; ESI-HRMS calcd for $\mathrm{C}_{8} \mathrm{H}_{10} \mathrm{ClS}^{+}[\mathrm{M}-\mathrm{OTf}]^{+}:$173.0186, found 173.0185 .<smiles></smiles>

$1 \mathrm{k}$

(4-bromophenyl)dimethylsulfonium OTf (1k). White solid (1.5 g, 86\%); mp: 125-126 ${ }^{\circ} \mathrm{C} ;{ }^{1} \mathrm{H}$ NMR (300 MHz, DMSO- $\left.d_{6}\right): \delta=8.01(\mathrm{~d}, J=9.0 \mathrm{~Hz}, 2 \mathrm{H}), 7.96(\mathrm{~d}, J=9.0 \mathrm{~Hz}, 2 \mathrm{H}), 3.26(\mathrm{~s}, 6 \mathrm{H})$; ${ }^{13} \mathrm{C}$ NMR $\left(101 \mathrm{MHz}\right.$, DMSO- $\left.d_{6}\right): \delta=133.8,132.4,128.2,126.7,28.6$; ESI-HRMS calcd for $\mathrm{C}_{8} \mathrm{H}_{10} \mathrm{BrS}^{+}$[M-OTf]: 216.9681, found 216.9677.<smiles>C[SH](C)c1ccc(C(F)(F)F)cc1</smiles>

11

(4-(trifluoromethyl)phenyl)dimethylsulfonium OTf (11). White solid (1.5 g, 88\%); mp: 129-130 ${ }^{\circ} \mathrm{C} ;{ }^{1} \mathrm{H}$ NMR $\left(300 \mathrm{MHz}, \mathrm{DMSO}-d_{6}\right): \delta=8.30(\mathrm{~d}, J=8.4 \mathrm{~Hz}, 2 \mathrm{H}), 8.14(\mathrm{~d}, J=8.5 \mathrm{~Hz}, 2 \mathrm{H}), 3.32(\mathrm{~s}$, $6 \mathrm{H}) .{ }^{13} \mathrm{C}$ NMR (101 MHz, DMSO- $\left.d_{6}\right): \delta=133.73$ (q, $\left.J=32.6 \mathrm{~Hz}\right), 132.40,131.42,127.50$ (q, $J=$ $3.7 \mathrm{~Hz}), 123.70$ (q, $J=270.7 \mathrm{~Hz}), 121.09$ (q, $J=320.2 \mathrm{~Hz}) ;{ }^{19} \mathrm{~F}$ NMR $\left(282 \mathrm{MHz}\right.$, DMSO- $\left.d_{6}\right): \delta=$ -62.2, -78.0; ESI-HRMS calcd for $\mathrm{C}_{9} \mathrm{H}_{10} \mathrm{~F}_{3} \mathrm{~S}^{+}$[M-OTf] ${ }^{+}$: 207.0450, found 207.0450.<smiles>C[As](C)c1ccc(O[AsH3])cc1</smiles>

(4-(tosyloxy)phenyl)dimethyl sulfonium OTf (1m). White solid (1.9 g, 86\%); mp: 141-143 ${ }^{\circ} \mathrm{C} ;{ }^{1} \mathrm{H}$ NMR $\left(300 \mathrm{MHz}\right.$, DMSO- $\left.d_{6}\right) \delta=8.11(\mathrm{~d}, J=8.9 \mathrm{~Hz}, 2 \mathrm{H}), 7.82(\mathrm{~d}, J=8.4 \mathrm{~Hz}, 2 \mathrm{H}), 7.52(\mathrm{~d}, J=8.1$ $\mathrm{Hz}, 2 \mathrm{H}), 7.43(\mathrm{~d}, J=8.9 \mathrm{~Hz}, 2 \mathrm{H}), 3.24(\mathrm{~s}, 6 \mathrm{H}), 2.44(\mathrm{~s}, 3 \mathrm{H}) ;{ }^{13} \mathrm{C}$ NMR $\left(75 \mathrm{MHz}, \mathrm{DMSO}-d_{6}\right): \delta=$ 152.7, 146.8, 132.7, 131.5, 130.9, 128.7, 126.3, 124.5, 28.7, 21.7; ESI-HRMS calcd for $\mathrm{C}_{15} \mathrm{H}_{17} \mathrm{O}_{3} \mathrm{~S}_{2}{ }^{+}$ [M-OTf] $]^{+}$309.0614, found 309.0620.<smiles>Cc1cccc([S+](C)C)c1</smiles>

1n

(m-tolyl)dimethyl sulfonium OTf (1n). White solid (1.2g, 85\%); mp: 81-82 ${ }^{\circ} \mathrm{C}$; ${ }^{1} \mathrm{H}$ NMR $(300$ MHz, DMSO- $\left.d_{6}\right): \delta=7.91(\mathrm{~s}, 1 \mathrm{H}), 7.89-7.81(\mathrm{~m}, 1 \mathrm{H}), 7.61(\mathrm{~d}, J=5.3 \mathrm{~Hz}, 2 \mathrm{H}), 3.25(\mathrm{~s}, 6 \mathrm{H}), 2.43$ (s, 3H); ${ }^{13} \mathrm{C}$ NMR (75 MHz, DMSO- $d_{6}$ ): $\delta=140.9,134.8,130.7,130.3,127.4,126.9,28.6,21.2$; ESI-HRMS calcd for $\mathrm{C}_{9} \mathrm{H}_{13} \mathrm{~S}^{+}[\mathrm{M}-\mathrm{OTf}]^{+}: 153.0732$, found 153.0729 . 


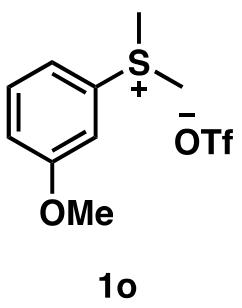

(3-methoxyphenyl)dimethylsulfonium OTf (1o). White solid (1.3g, 83\%); mp: 99-101 ${ }^{\circ} \mathrm{C} ;{ }^{1} \mathrm{H}$ NMR (300 MHz, DMSO- $\left.d_{6}\right): \delta=7.68-7.57(\mathrm{~m}, 3 \mathrm{H}), 7.35$ (d, $\left.J=7.4 \mathrm{~Hz}, 1 \mathrm{H}\right), 3.88(\mathrm{~s}, 3 \mathrm{H}), 3.27$ (s, $6 \mathrm{H}) ;{ }^{13} \mathrm{C}$ NMR $\left(101 \mathrm{MHz}, \mathrm{DMSO}-d_{6}\right): \delta=160.7,131.9,128.1,122.2,120.1,115.3,56.4,28.5$; ESIHRMS calcd for $\mathrm{C}_{9} \mathrm{H}_{13} \mathrm{OS}^{+}[\mathrm{M}-\mathrm{OTf}]^{+}: 169.0682$, found 169.0678 .<smiles>C[S+](C)c1cccc(F)c1</smiles>

$1 p$

(3-fluorophenyl)dimethylsulfonium OTf (1p). White solid (1.2 g, 82\%); mp: 93-94 ${ }^{\circ} \mathrm{C}$; ${ }^{1} \mathrm{H}$ NMR $\left(300 \mathrm{MHz}, \mathrm{DMSO}-d_{6}\right): \delta=8.07(\mathrm{~d}, J=8.9 \mathrm{~Hz}, 1 \mathrm{H}), 7.90(\mathrm{~d}, J=7.9 \mathrm{~Hz}, 1 \mathrm{H}), 7.84-7.72(\mathrm{~m}, 1 \mathrm{H})$, $7.66(\mathrm{t}, J=8.4 \mathrm{~Hz}, 1 \mathrm{H}), 3.28(\mathrm{~s}, 6 \mathrm{H}) ;{ }^{13} \mathrm{C} \mathrm{NMR}\left(75 \mathrm{MHz}, \mathrm{DMSO}-d_{6}\right) \delta=162.6(\mathrm{~d}, J=249.3 \mathrm{~Hz})$, 132.9 (d, $J=8.3 \mathrm{~Hz}), 129.2$ (d, $J=8.4 \mathrm{~Hz}), 127.0$ (d, $J=3.3 \mathrm{~Hz}), 121.3$ (d, $J=21.0 \mathrm{~Hz}), 117.4$ (d, $J=25.6 \mathrm{~Hz}), 28.6 ;{ }^{19} \mathrm{~F}$ NMR (282 MHz, DMSO- $\left.d_{6}\right) \delta=-77.76,-108.95$. ESI-HRMS calcd for $\mathrm{C}_{8} \mathrm{H}_{10} \mathrm{FS}^{+}[\mathrm{M}-\mathrm{OTf}]^{+}: 157.0482$, found 157.0482 .<smiles>C[S+](C)c1cccc(Cl)c1</smiles>

$1 q$

(3-chlorophenyl)dimethylsulfonium OTf (1q). White solid (1.3 g, 82\%); mp: 92-93 ${ }^{\circ} \mathrm{C}$; ${ }^{1} \mathrm{H}$ NMR $\left(300 \mathrm{MHz}, \mathrm{DMSO}-d_{6}\right): \delta=8.23(\mathrm{~s}, 1 \mathrm{H}), 8.02(\mathrm{~d}, J=8.7 \mathrm{~Hz}, 1 \mathrm{H}), 7.86(\mathrm{~d}, J=7.2 \mathrm{~Hz}, 1 \mathrm{H}), 7.74(\mathrm{t}$, $J=8.0 \mathrm{~Hz}, 1 \mathrm{H}), 3.29(\mathrm{~s}, 6 \mathrm{H}) ;{ }^{13} \mathrm{C}$ NMR $\left(75 \mathrm{MHz}, \mathrm{DMSO}-d_{6}\right): \delta=135.1,134.0,132.4,129.9,129.4$, 129.3, 28.6; ESI-HRMS calcd for $\mathrm{C}_{8} \mathrm{H}_{10} \mathrm{ClS}^{+}$[M-OTf] ${ }^{+}:$173.0186, found 173.0185.<smiles>C[S+](C)c1cccc(Br)c1</smiles>

$1 r$

(3-bromophenyl)dimethylsulfonium OTf (1r). White solid (1.5 g, 87\%); mp: 83-84 ${ }^{\circ} \mathrm{C}$; ${ }^{1} \mathrm{H}$ NMR (300 MHz, DMSO-d $): \delta=8.35(\mathrm{~s}, 1 \mathrm{H}), 8.07(\mathrm{~d}, J=7.3 \mathrm{~Hz}, 1 \mathrm{H}), 8.00(\mathrm{~d}, J=8.1 \mathrm{~Hz}, 1 \mathrm{H}), 7.67$ (t, 
$J=8.1 \mathrm{~Hz}, 1 \mathrm{H}), 3.29(\mathrm{~s}, 6 \mathrm{H}) ;{ }^{13} \mathrm{C}$ NMR (75 MHz, DMSO- $\left.d_{6}\right): \delta=136.9,132.6,132.6,129.6,129.5$, 123.4, 28.6; ESI-HRMS calcd for $\mathrm{C}_{8} \mathrm{H}_{10} \mathrm{BrS}^{+}$[M-OTf] $^{+}: 216.9681$, found 216.9678 .<smiles>C[S+](C)c1cccc(C(F)(F)F)c1</smiles>

$1 \mathrm{~s}$

(3-(trifluoromethyl)phenyl)dimethyl sulfonium OTf (1s). White solid (1.6 g, 93\%); mp: 121-123 ${ }^{\circ} \mathrm{C} ;{ }^{1} \mathrm{H}$ NMR $\left(300 \mathrm{MHz}\right.$, DMSO- $\left.d_{6}\right) \delta=8.50(\mathrm{~s}, 1 \mathrm{H}), 8.38(\mathrm{~d}, J=8.2 \mathrm{~Hz}, 1 \mathrm{H}), 8.17(\mathrm{~d}, J=7.9 \mathrm{~Hz}$, $1 \mathrm{H}), 7.97(\mathrm{t}, J=8.0 \mathrm{~Hz}, 1 \mathrm{H}), 3.32(\mathrm{~s}, 6 \mathrm{H}) .{ }^{13} \mathrm{C}$ NMR (101 MHz, DMSO- $\left.d_{6}\right) \delta=134.5,132.0,131.2$ $(\mathrm{q}, J=33.0 \mathrm{~Hz}), 130.6(\mathrm{q}, J=3.6 \mathrm{~Hz}), 129.1,127.7$ (q, $J=3.8 \mathrm{~Hz}), 121.1(\mathrm{q}, J=323.2 \mathrm{~Hz}), 123.6(\mathrm{q}$, $J=323.2 \mathrm{~Hz}), 28.4 .{ }^{19} \mathrm{~F}$ NMR $\left(282 \mathrm{MHz}, \mathrm{DMSO}-d_{6}\right) \delta=-61.68,-78.01$; ESI-HRMS calcd for $\mathrm{C}_{9} \mathrm{H}_{10} \mathrm{~F}_{3} \mathrm{~S}^{+}$[M-OTf] $]^{+}: 207.0450$, found 207.0450.<smiles>C[S+](C)c1cccc(C(=O)O)c1</smiles>

1t

(3-carboxyphenyl)dimethylsulfonium OTf (1t). White solid (1.2 g, 80\%); mp: 151-153 ${ }^{\circ} \mathrm{C} ;{ }^{1} \mathrm{H}$ NMR (300 MHz, DMSO- $\left.d_{6}\right): \delta=8.56(\mathrm{~s}, 1 \mathrm{H}), 8.30(\mathrm{dd}, J=13.5,8.4 \mathrm{~Hz}, 2 \mathrm{H}), 7.86(\mathrm{t}, J=7.9 \mathrm{~Hz}$, 1H), 3.30 (s, 6H); ${ }^{13} \mathrm{C}$ NMR (101 MHz, DMSO- $\left.d_{6}\right): \delta=166.2,134.4,134.2,133.2,131.4,131.3$, 128.3, 28.5; ESI-HRMS calcd for $\mathrm{C}_{9} \mathrm{H}_{11} \mathrm{O}_{2} \mathrm{~S}^{+}$[M-OTf] $]^{+}$183.0474, found 183.0471.<smiles>CCOC(=O)c1cccc([S+](C)=C[Bi])c1</smiles>

1u

(3-(ethoxycarbonyl)phenyl)dimethylsulfonium OTf (1u). White solid (1.2 g, 82\%); mp: 99-100 ${ }^{\circ} \mathrm{C} ;{ }^{1} \mathrm{H}$ NMR (300 MHz, DMSO- $\left.d_{6}\right): \delta=8.56(\mathrm{~s}, 1 \mathrm{H}), 8.37(\mathrm{~d}, J=8.0 \mathrm{~Hz}, 1 \mathrm{H}), 8.33(\mathrm{~d}, J=8.1 \mathrm{~Hz}$, $1 \mathrm{H}), 7.89(\mathrm{t}, J=7.9 \mathrm{~Hz}, 1 \mathrm{H}), 4.40(\mathrm{q}, J=7.1 \mathrm{~Hz}, 2 \mathrm{H}), 3.30(\mathrm{~s}, 6 \mathrm{H}), 1.36(\mathrm{t}, J=7.1 \mathrm{~Hz}, 3 \mathrm{H}) ;{ }^{13} \mathrm{C}$ NMR (75 MHz, DMSO- $\left.d_{6}\right): \delta=164.7,134.5,134.3,132.1,131.5,131.4,128.5,62.1,28.5,14.6$; ESI-HRMS calcd for $\mathrm{C}_{11} \mathrm{H}_{15} \mathrm{O}_{2} \mathrm{~S}^{+}$[M-OTf] $]^{+}: 211.0787$, found 211.0787.<smiles>Cc1ccccc1S(C)=O</smiles>

$1 v$ 
(o-tolyl)dimethyl sulfonium OTf (1v). White solid (1.3 g, 88\%); mp: 86-88 ${ }^{\circ} \mathrm{C} ;{ }^{1} \mathrm{H}$ NMR $(300$ MHz, DMSO- $\left.d_{6}\right): \delta=8.13(\mathrm{~d}, J=7.9 \mathrm{~Hz}, 1 \mathrm{H}), 7.71-7.64(\mathrm{~m}, 1 \mathrm{H}), 7.60(\mathrm{t}, J=7.5 \mathrm{~Hz}, 1 \mathrm{H}), 7.53(\mathrm{~d}$, $J=7.8 \mathrm{~Hz}, 1 \mathrm{H}), 3.25(\mathrm{~s}, 6 \mathrm{H}), 2.61(\mathrm{~s}, 3 \mathrm{H}) ;{ }^{13} \mathrm{C}$ NMR $\left(101 \mathrm{MHz}, \mathrm{DMSO}-d_{6}\right): \delta=141.3,134.0$, 132.1, 129.0, 128.6, 126.4, 28.2, 19.8; ESI-HRMS calcd for $\mathrm{C}_{9} \mathrm{H}_{13} \mathrm{~S}^{+}[\mathrm{M}-\mathrm{OTf}]^{+}: 153.0732$, found 153.0731 .<smiles>C[SH](C)c1ccccc1O</smiles>

$1 w$

(2-hydroxyphenyl)dimethylsulfonium OTf (1w). White solid (1.2 g, 75\%); mp: 76-78 ${ }^{\circ} \mathrm{C} ;{ }^{1} \mathrm{H}$ NMR (300 MHz, DMSO- $\left.d_{6}\right): \delta=11.71(\mathrm{~s}, 1 \mathrm{H}), 7.75(\mathrm{~d}, J=8.2 \mathrm{~Hz}, 1 \mathrm{H}), 7.63-7.53(\mathrm{~m}, 1 \mathrm{H}), 7.12$ (dt, $J=8.3,4.2 \mathrm{~Hz}, 2 \mathrm{H}), 3.22(\mathrm{~s}, 6 \mathrm{H}) ;{ }^{13} \mathrm{C}$ NMR (101 MHz, DMSO- $\left.d_{6}\right) \delta=157.8,135.7,130.1$, 121.1, 117.3, 111.1, 26.2; ESI-HRMS calcd for $\mathrm{C}_{8} \mathrm{H}_{10} \mathrm{OS}^{+}[\mathrm{M}-\mathrm{OTf}]^{+}$: 155.0525 , found 155.0528.<smiles>COc1ccccc1/S(C)=C/[O]</smiles>

$1 \mathrm{x}$

(2-methoxyphenyl)dimethylsulfonium OTf (1x). White solid (1.2 g, 75\%); mp: $77-78{ }^{\circ} \mathrm{C} ;{ }^{1} \mathrm{H}$ NMR (300 MHz, DMSO- $\left.d_{6}\right): \delta=7.85(\mathrm{~d}, J=8.0 \mathrm{~Hz}, 1 \mathrm{H}), 7.76(\mathrm{t}, J=7.9 \mathrm{~Hz}, 1 \mathrm{H}), 7.38(\mathrm{~d}, J=8.4$ $\mathrm{Hz}, 1 \mathrm{H}), 7.28(\mathrm{t}, J=7.7 \mathrm{~Hz}, 1 \mathrm{H}), 4.00(\mathrm{~s}, 3 \mathrm{H}), 3.22(\mathrm{~s}, 6 \mathrm{H}) ;{ }^{13} \mathrm{C}$ NMR $\left(75 \mathrm{MHz}\right.$, DMSO-$\left.d_{6}\right) \delta 158.4$, 135.9, 130.1, 122.6, 113.6, 113.6, 57.4, 26.6; ESI-HRMS calcd for $\mathrm{C}_{9} \mathrm{H}_{13} \mathrm{OS}^{+}$[M-OTf] ${ }^{+}$: 169.0682, found 169.0678 .<smiles>C[SH](C)c1ccccc1F</smiles>

$1 y$

(2-fluorophenyl)dimethylsulfonium OTf (1y). White solid (1.1 g, 72\%); mp: 68-69 ${ }^{\circ} \mathrm{C}$; ${ }^{1} \mathrm{H}$ NMR (300 MHz, DMSO- $\left.d_{6}\right): \delta=8.22-8.13(\mathrm{~m}, 1 \mathrm{H}), 7.93-7.81(\mathrm{~m}, 1 \mathrm{H}), 7.71-7.54(\mathrm{~m}, 2 \mathrm{H}), 3.32(\mathrm{~s}, 6 \mathrm{H})$; ${ }^{13} \mathrm{C}$ NMR $\left(75 \mathrm{MHz}\right.$, DMSO- $\left.d_{6}\right) \delta=161.0(\mathrm{~d}, J=253.4 \mathrm{~Hz}), 137.0(\mathrm{~d}, J=8.8 \mathrm{~Hz}), 131.0,127.2(\mathrm{~d}$, $J=3.3 \mathrm{~Hz}), 117.8(\mathrm{~d}, J=20.4 \mathrm{~Hz}), 114.5(\mathrm{~d}, J=14.3 \mathrm{~Hz}) ;{ }^{19} \mathrm{~F}$ NMR $\left(282 \mathrm{MHz}\right.$, DMSO- $\left.d_{6}\right) \delta=-$ 77.78, -113.03; ESI-HRMS calcd for $\mathrm{C}_{8} \mathrm{H}_{10} \mathrm{FS}^{+}$[M-OTf] ${ }^{+}$: 157.0482, found 157.0479.<smiles>C[SH](C)c1ccccc1Cl</smiles>

12 
(2-chlorophenyl)dimethylsulfonium OTf (1z). White solid (1.2 g, 75\%); mp: 96-98 ${ }^{\circ} \mathrm{C} ;{ }^{1} \mathrm{H}$ NMR (300 MHz, DMSO- $\left.d_{6}\right): \delta=8.23-8.12(\mathrm{~m}, 1 \mathrm{H}), 7.88-7.82(\mathrm{~m}, 1 \mathrm{H}), 7.82-7.72(\mathrm{~m}, 2 \mathrm{H}), 3.32(\mathrm{~s}, 6 \mathrm{H})$; ${ }^{13} \mathrm{C}$ NMR (75 MHz, DMSO- $\left.d_{6}\right): \delta=135.6,135.0,131.4,130.5,130.1,126.2,27.7$; ESI-HRMS calcd for $\mathrm{C}_{8} \mathrm{H}_{10} \mathrm{ClS}^{+}[\mathrm{M}-\mathrm{OTf}]^{+}:$173.0186, found 173.0185.<smiles>Brc1ccccc1Br</smiles>

2a

4,4,5,5-tetramethyl-2-phenyl-1,3,2-dioxaborolane (2a). ${ }^{5}$ Pale yellow oil $(15.5 \mathrm{mg}, 67 \%) ;{ }^{1} \mathrm{H}$ $\operatorname{NMR}\left(300 \mathrm{MHz}, \mathrm{CDCl}_{3}\right): \delta=7.81(\mathrm{~d}, J=6.8 \mathrm{~Hz}, 2 \mathrm{H}), 7.46(\mathrm{t}, J=7.3 \mathrm{~Hz}, 1 \mathrm{H}), 7.36(\mathrm{t}, J=7.2 \mathrm{~Hz}$, 2H), 1.35 (s, 12H); ${ }^{13} \mathrm{C}$ NMR (75 MHz, $\left.\mathrm{CDCl}_{3}\right): \delta=134.8,131.3,127.8,83.8,24.9$; The carbon which was attached to the boron atom was not detected due to quadrupolar broadening. GC-MS $\mathrm{M}^{+}$ $\mathrm{m} / \mathrm{z} 204.1$.<smiles>Cc1ccc(Br)cc1</smiles>

2b

4,4,5,5-tetramethyl-2-(p-tolyl)-1,3,2-dioxaborolane (2b). ${ }^{6}$ Pale yellow oil $(17.6 \mathrm{mg}, 81 \%)$; ${ }^{1} \mathrm{H}$ $\operatorname{NMR}\left(300 \mathrm{MHz}, \mathrm{CDCl}_{3}\right): \delta=7.70(\mathrm{~d}, J=7.1 \mathrm{~Hz}, 2 \mathrm{H}), 7.18(\mathrm{~d}, J=7.2 \mathrm{~Hz}, 2 \mathrm{H}), 2.36$ (s, 3H), 1.33 (s, 12H).; ${ }^{13} \mathrm{C} \mathrm{NMR}\left(101 \mathrm{MHz}, \mathrm{CDCl}_{3}\right): \delta=141.4,134.8,128.6,83.6,24.9,21.8$; The carbon which was attached to the boron atom was not detected due to quadrupolar broadening. GC-MS M+ $\mathrm{m} / \mathrm{z}$ 218.1.<smiles>CC(C)(C)c1ccc(Br)cc1</smiles>

2-(4-(tert-butyl)phenyl)-4,4,5,5-tetramethyl-1,3,2-dioxaborolane (2c). ${ }^{7}$ White solid (19.7 mg, $76 \%) ;{ }^{1} \mathrm{H}$ NMR (300 MHz, $\left.\mathrm{CDCl}_{3}\right): \delta=7.76(\mathrm{~d}, J=8.1 \mathrm{~Hz}, 2 \mathrm{H}), 7.41(\mathrm{~d}, J=8.1 \mathrm{~Hz}, 2 \mathrm{H}), 1.33$ (s, 12H), 1.32 (s, 9H); ${ }^{13} \mathrm{C}$ NMR (101 MHz, $\left.\mathrm{CDCl}_{3}\right): \delta=154.5,134.7,124.7,83.6,34.9,31.2$, 24.9; The carbon which was attached to the boron atom was not detected due to quadrupolar broadening. GC-MS M+ m/z 260.2.<smiles>Oc1ccc(Br)cc1</smiles>

2d

4-(4,4,5,5-tetramethyl-1,3,2-dioxaborolan-2-yl)phenol (2d). ${ }^{11}$ White solid $(18.0 \mathrm{mg}, 82 \%) ;{ }^{1} \mathrm{H}$ $\operatorname{NMR}\left(300 \mathrm{MHz}, \mathrm{CDCl}_{3}\right): \delta=7.70(\mathrm{~d}, J=8.5 \mathrm{~Hz}, 2 \mathrm{H}), 6.82(\mathrm{~d}, J=8.6 \mathrm{~Hz}, 2 \mathrm{H}), 5.63(\mathrm{~s}, 1 \mathrm{H}), 1.33$ (s, $12 \mathrm{H}) ;{ }^{13} \mathrm{C}$ NMR $\left(75 \mathrm{MHz}, \mathrm{CDCl}_{3}\right): \delta=158.6,136.8,114.9,83.7,24.8$; The carbon which was attached to the boron atom was not detected due to quadrupolar broadening. GC-MS M+ m/z 220.1. 
<smiles>COc1ccc(Br)cc1</smiles>

$2 e$

2-(4-methoxyphenyl)-4,4,5,5-tetramethyl-1,3,2-dioxaborolane (2e). ${ }^{5}$ White solid (15.9 $\mathrm{mg}$, $68 \%$ ); ${ }^{1} \mathrm{H}$ NMR (300 MHz, $\left.\mathrm{CDCl}_{3}\right): \delta=7.75(\mathrm{~d}, J=8.3 \mathrm{~Hz}, 2 \mathrm{H}), 6.90(\mathrm{~d}, J=8.3 \mathrm{~Hz}, 2 \mathrm{H}), 3.82(\mathrm{~s}$, $3 \mathrm{H}), 1.33(\mathrm{~s}, 12 \mathrm{H}) ;{ }^{13} \mathrm{C} \mathrm{NMR}\left(75 \mathrm{MHz}, \mathrm{CDCl}_{3}\right): \delta=162.2,136.5,113.3,83.6,55.1,24.9$; The carbon which was attached to the boron atom was not detected due to quadrupolar broadening. GC$\mathrm{MS} \mathrm{M}^{+} \mathrm{m} / \mathrm{z} 234.1$.<smiles>C=CCOc1ccc(Br)cc1</smiles>

$2 f$

2-(4-(allyloxy)phenyl)-4,4,5,5-tetramethyl-1,3,2-dioxaborolane (2f). ${ }^{8}$ White solid (18.2 $\mathrm{mg}$, $70 \%$ ); ${ }^{1} \mathrm{H}$ NMR (300 MHz, $\mathrm{CDCl}_{3}$ ): $\delta=7.74(\mathrm{dd}, J=8.6,2 \mathrm{H}), 6.91(\mathrm{dd}, J=8.6,2 \mathrm{H}), 6.05$ (ddt, $J=$ $17.2,10.5,5.2 \mathrm{~Hz}, 1 \mathrm{H}), 5.41(\mathrm{dq}, J=17.3,1.6 \mathrm{~Hz}, 1 \mathrm{H}), 5.28(\mathrm{dq}, J=10.5,1.4 \mathrm{~Hz}, 1 \mathrm{H}), 4.56(\mathrm{dt}, J$ $=5.3,1.5 \mathrm{~Hz}, 2 \mathrm{H}), 1.33(\mathrm{~s}, 12 \mathrm{H}) ;{ }^{13} \mathrm{C} \mathrm{NMR}\left(75 \mathrm{MHz}, \mathrm{CDCl}_{3}\right): \delta=161.2,136.5,133.1,117.7,114.1$, 83.6, 68.5, 24.9; The carbon which was attached to the boron atom was not detected due to quadrupolar broadening. GC-MS M+ m/z 260.1.<smiles>CC1(C)COC(c2ccc(Br)cc2)OC1</smiles>

$2 \mathrm{~g}$

2-(4-(5,5-dimethyl-1,3-dioxan-2-yl)phenyl)-4,4,5,5-tetramethyl-1,3,2-dioxaborolane

$(\mathbf{2 g}) .^{12}$ White solid (21.0 mg, 66\%); ${ }^{1} \mathrm{H}$ NMR (300 MHz, $\left.\mathrm{CDCl}_{3}\right): \delta=7.81(\mathrm{~d}, J=8.1 \mathrm{~Hz}, 2 \mathrm{H}), 7.50(\mathrm{~d}, J=$ $7.9 \mathrm{~Hz}, 2 \mathrm{H}), 5.39$ (s, 1H), 3.77 (d, $J=11.2 \mathrm{~Hz}, 2 \mathrm{H}), 3.64$ (d, $J=10.6 \mathrm{~Hz}, 2 \mathrm{H}), 1.34$ (s, 12H), 1.29 (s, 3H), 0.80 (s, 3H); ${ }^{13} \mathrm{C} \mathrm{NMR} \mathrm{(75} \mathrm{MHz,} \mathrm{CDCl}_{3}$ ): $\delta=141.3,134.8,125.4,101.6,83.8,77.7,30.3,24.9$, 23.1, 21.9; The carbon which was attached to the boron atom was not detected due to quadrupolar broadening. GC-MS M+ m/z 318.2.<smiles>CC1(C)COC(C)(c2ccc(Br)cc2)OC1</smiles>

$2 \mathrm{~h}$

4,4,5,5-tetramethyl-2-(4-(2,5,5-trimethyl-1,3-dioxan-2-yl)phenyl)-1,3,2-dioxaborolane $(2 \mathrm{~h}) \mathbf{.}^{12}$ White solid (21.3 mg, 64\%); ${ }^{1} \mathrm{H}$ NMR (300 MHz, $\left.\mathrm{CDCl}_{3}\right): \delta=7.85$ (d, $\left.J=8.2 \mathrm{~Hz}, 2 \mathrm{H}\right), 7.44(\mathrm{~d}, J=$ $8.2 \mathrm{~Hz}, 2 \mathrm{H}), 3.47-3.32(\mathrm{~m}, 4 \mathrm{H}), 1.53$ (s, 3H), $1.35(\mathrm{~s}, 12 \mathrm{H}), 1.27$ (s, 3H), $0.56(\mathrm{~s}, 3 \mathrm{H}) .{ }^{13} \mathrm{C}$ NMR $(75$ $\left.\mathrm{MHz}, \mathrm{CDCl}_{3}\right): \delta=144.1,135.1,126.2,100.3,83.9,71.8,32.0,29.9,24.9,22.9,21.8$; The carbon 
which was attached to the boron atom was not detected due to quadrupolar broadening. GC-MS $\mathrm{M}^{+}$ $\mathrm{m} / \mathrm{z} 332.2$.<smiles>Fc1ccc(Br)cc1</smiles>

2i

2-(4-fluorophenyl)-4,4,5,5-tetramethyl-1,3,2-dioxaborolane (2i). ${ }^{5}$ Yellow oil (15.7 mg, 71\%); ${ }^{1} \mathrm{H}$ NMR (300 MHz, CDCl $): \delta=7.80(\mathrm{~m}, 2 \mathrm{H}), 7.09-6.99(\mathrm{~m}, 2 \mathrm{H}), 1.33(\mathrm{~s}, 12 \mathrm{H}) ;{ }^{13} \mathrm{C} \mathrm{NMR}(75 \mathrm{MHz}$, $\left.\mathrm{CDCl}_{3}\right): \delta=165.1(\mathrm{~d}, J=250.3 \mathrm{~Hz}), 137.1(\mathrm{~d}, J=8.2 \mathrm{~Hz}), 114.9(\mathrm{~d}, J=20.2 \mathrm{~Hz}), 83.9,24.9 ;{ }^{19} \mathrm{~F}$ $\operatorname{NMR}\left(282 \mathrm{MHz}, \mathrm{CDCl}_{3}\right): \delta=-108.35$. The carbon which was attached to the boron atom was not detected due to quadrupolar broadening. GC-MS M+ $\mathrm{m} / \mathrm{z} 222.1$.<smiles>Clc1ccc(Br)cc1</smiles>

$2 \mathrm{j}$

2-(4-chlorophenyl)-4,4,5,5-tetramethyl-1,3,2-dioxaborolane (2j). ${ }^{5}$ Colorless oil (18.3 mg, 77\%); ${ }^{1} \mathrm{H}$ NMR (300 MHz, $\left.\mathrm{CDCl}_{3}\right): \delta=7.72(\mathrm{~d}, J=7.2 \mathrm{~Hz}, 2 \mathrm{H}), 7.33(\mathrm{~d}, J=7.2 \mathrm{~Hz}, 2 \mathrm{H}), 1.33(\mathrm{~s}, 12 \mathrm{H})$; ${ }^{13} \mathrm{C}$ NMR (75 MHz, $\mathrm{CDCl}_{3}$ ): $\delta=137.6,136.2,128.0,84.0,24.9$; The carbon which was attached to the boron atom was not detected due to quadrupolar broadening. GC-MS $\mathrm{M}^{+} \mathrm{m} / \mathrm{z}$ 238.1.<smiles>Brc1ccc(Br)cc1</smiles>

2k

2-(4-bromophenyl)-4,4,5,5-tetramethyl-1,3,2-dioxaborolane (2k). ${ }^{5}$ Colorless oil (14.9 mg, 53\%); ${ }^{1} \mathrm{H} \mathrm{NMR}\left(300 \mathrm{MHz}, \mathrm{CDCl}_{3}\right): \delta=7.66(\mathrm{~d}, J=8.1 \mathrm{~Hz}, 2 \mathrm{H}), 7.50(\mathrm{~d}, J=8.2 \mathrm{~Hz}, 2 \mathrm{H}), 1.34(\mathrm{~s}, 12 \mathrm{H})$; ${ }^{13} \mathrm{C}$ NMR $\left(75 \mathrm{MHz}, \mathrm{CDCl}_{3}\right): \delta=136.3,130.9,126.2,84.1,24.9$; The carbon which was attached to the boron atom was not detected due to quadrupolar broadening. GC-MS $\mathrm{M}^{+} \mathrm{m} / \mathrm{z}$ 282.0.<smiles>FC(F)(F)c1ccc(Br)cc1</smiles>

2I

4,4,5,5-tetramethyl-2-(4-(trifluoromethyl)phenyl)-1,3,2-dioxaborolane (2I). ${ }^{6}$ White solid (11.9 mg, 44\%); ${ }^{1} \mathrm{H}$ NMR (300 MHz, $\left.\mathrm{CDCl}_{3}\right): \delta=7.90-7.92(\mathrm{~m}, 2 \mathrm{H}), 7.59-7.62(\mathrm{~m}, 2 \mathrm{H}), 1.35(\mathrm{~s}, 12 \mathrm{H})$; ${ }^{13} \mathrm{C}$ NMR (75 MHz, $\left.\mathrm{CDCl}_{3}\right): \delta=135.1,132.8(\mathrm{q}, J=32.0 \mathrm{~Hz}), 124.3(\mathrm{q}, J=3.8 \mathrm{~Hz}), 124.2(\mathrm{q}, J=$ $270.7 \mathrm{~Hz}), 84.2,24.7 ;{ }^{19} \mathrm{~F}$ NMR $\left(282 \mathrm{MHz}, \mathrm{CDCl}_{3}\right): \delta=-63.08$. The carbon which was attached to the boron atom was not detected due to quadrupolar broadening. GC-MS M+ m/z 272.1. 
TsO

$2 m$

4-(4,4,5,5-tetramethyl-1,3,2-dioxaborolan-2-yl)phenyl 4-methylbenzenesulfonate ( $2 \mathrm{~m}) .{ }^{9}$ White solid (23.9 mg, 64\%); ${ }^{1} \mathrm{H}$ NMR (300 MHz, $\left.\mathrm{CDCl}_{3}\right): \delta=7.71(\mathrm{~d}, J=9.0 \mathrm{~Hz}, 2 \mathrm{H}), 7.68(\mathrm{~d}, J=8.4$ $\mathrm{Hz}, 2 \mathrm{H}), 7.28(\mathrm{~d}, J=8.4 \mathrm{~Hz}, 2 \mathrm{H}), 6.97(\mathrm{~d}, J=9.0 \mathrm{~Hz}, 2 \mathrm{H}), 2.43(\mathrm{~s}, 3 \mathrm{H}), 1.32(\mathrm{~s}, 12 \mathrm{H}) ;{ }^{13} \mathrm{C} \mathrm{NMR}$ $\left(101 \mathrm{MHz}, \mathrm{CDCl}_{3}\right): \delta=152.1,145.5,136.4,132.5,129.9,128.6,121.8,84.2,25.0,21.8$; The carbon which was attached to the boron atom was not detected due to quadrupolar broadening.<smiles>Cc1cccc(Br)c1</smiles>

2n

4,4,5,5-tetramethyl-2-(m-tolyl)-1,3,2-dioxaborolane (2n). ${ }^{10}$ Colorless oil $(17.8 \mathrm{mg}, 82 \%) ;{ }^{1} \mathrm{H}$ $\operatorname{NMR}\left(300 \mathrm{MHz}, \mathrm{CDCl}_{3}\right): \delta=7.64(\mathrm{~s}, 1 \mathrm{H}), 7.60(\mathrm{t}, J=3.8 \mathrm{~Hz}, 1 \mathrm{H}), 7.28-7.25(\mathrm{~m}, 2 \mathrm{H}), 2.35(\mathrm{~s}, 3 \mathrm{H})$, 1.34 (s, 12H); ${ }^{13} \mathrm{C}$ NMR (75 MHz, $\left.\mathrm{CDCl}_{3}\right): \delta=137.2,135.4,132.1,131.8,127.7,83.8,24.9,21.3$; The carbon which was attached to the boron atom was not detected due to quadrupolar broadening. GC-MS M+ m/z 218.1.<smiles>COc1cccc(Br)c1</smiles>

20

2-(3-methoxyphenyl)-4,4,5,5-tetramethyl-1,3,2-dioxaborolane (2o). ${ }^{10}$ Colorless oil (15.9 mg, $68 \%) ;{ }^{1} \mathrm{H}$ NMR $\left(300 \mathrm{MHz}, \mathrm{CDCl}_{3}\right): \delta=7.40(\mathrm{~d}, J=7.2 \mathrm{~Hz}, 1 \mathrm{H}), 7.33(\mathrm{~d}, J=2.6 \mathrm{~Hz}, 1 \mathrm{H}), 7.33-$ $7.26(\mathrm{~m}, 1 \mathrm{H}), 7.05-6.96(\mathrm{~m}, 1 \mathrm{H}), 3.82(\mathrm{~s}, 3 \mathrm{H}), 1.34(\mathrm{~s}, 12 \mathrm{H}) ;{ }^{13} \mathrm{C} \mathrm{NMR}\left(75 \mathrm{MHz}, \mathrm{CDCl}_{3}\right): \delta=159.1$, $129.0,127.2,118.7,117.9,83.9,55.3,24.9$; The carbon which was attached to the boron atom was not detected due to quadrupolar broadening. GC-MS M+ m/z 234.1.<smiles>Fc1cccc(Br)c1</smiles>

$2 p$

2-(3-fluorophenyl)-4,4,5,5-tetramethyl-1,3,2-dioxaborolane (2p). ${ }^{10}$ Colorless oil (14.4 mg, 65\%); ${ }^{1} \mathrm{H}$ NMR $\left(300 \mathrm{MHz}, \mathrm{CDCl}_{3}\right): \delta=7.57(\mathrm{~d}, J=7.1 \mathrm{~Hz}, 1 \mathrm{H}), 7.47(\mathrm{~d}, J=9.1 \mathrm{~Hz}, 1 \mathrm{H}), 7.34(\mathrm{q}, J=6.9$, $6.3 \mathrm{~Hz}, 1 \mathrm{H}), 7.13(\mathrm{t}, J=8.5 \mathrm{~Hz}, 1 \mathrm{H}), 1.34(\mathrm{~d}, J=2.4 \mathrm{~Hz}, 12 \mathrm{H}) ;{ }^{13} \mathrm{C} \mathrm{NMR}\left(75 \mathrm{MHz}, \mathrm{CDCl}_{3}\right): \delta=$ $162.5(\mathrm{~d}, J=246.2 \mathrm{~Hz}), 130.3(\mathrm{~d}, J=2.9 \mathrm{~Hz}), 129.5(\mathrm{~d}, J=7.3 \mathrm{~Hz}), 121.0(\mathrm{~d}, J=19.6 \mathrm{~Hz}), 118.2$ $(\mathrm{d}, J=20.8 \mathrm{~Hz}), 84.1,24.9 ;{ }^{19} \mathrm{~F} \mathrm{NMR}\left(282 \mathrm{MHz}, \mathrm{CDCl}_{3}\right): \delta=-114.20$. The carbon which was attached to the boron atom was not detected due to quadrupolar broadening. GC-MS M+ m/z 222.1. 
<smiles>Clc1cccc(Br)c1</smiles>

$2 q$

2-(3-chlorophenyl)-4,4,5,5-tetramethyl-1,3,2-dioxaborolane (2q). ${ }^{10}$ Yellow oil (14.2 mg, 60\%); ${ }^{1} \mathrm{H}$ NMR $\left(300 \mathrm{MHz}, \mathrm{CDCl}_{3}\right): \delta=7.78(\mathrm{~s}, 1 \mathrm{H}), 7.67(\mathrm{~d}, J=7.3 \mathrm{~Hz}, 1 \mathrm{H}), 7.42(\mathrm{~d}, J=8.1 \mathrm{~Hz}, 1 \mathrm{H})$, $7.30(\mathrm{t}, J=7.7 \mathrm{~Hz}, 1 \mathrm{H}), 1.34$ (s, $12 \mathrm{H}) ;{ }^{13} \mathrm{C}$ NMR $\left(75 \mathrm{MHz}, \mathrm{CDCl}_{3}\right)$ : 134.6, 134.0, 132.7, 131.3, 129.2, 84.1, 24.7; The carbon which was attached to the boron atom was not detected due to quadrupolar broadening. GC-MS $\mathrm{M}^{+} \mathrm{m} / \mathrm{z} 238.1$.<smiles>Brc1cccc(Br)c1</smiles>

$2 r$

2-(3-bromophenyl)-4,4,5,5-tetramethyl-1,3,2-dioxaborolane (2r). ${ }^{13}$ Colorless oil (14.3 mg, 51\%); ${ }^{1} \mathrm{H}$ NMR $\left(300 \mathrm{MHz}, \mathrm{CDCl}_{3}\right): \delta=7.94(\mathrm{~s}, 1 \mathrm{H}), 7.71(\mathrm{~d}, J=7.3 \mathrm{~Hz}, 1 \mathrm{H}), 7.57(\mathrm{~d}, J=7.8 \mathrm{~Hz}, 1 \mathrm{H})$, $7.23(\mathrm{t}, J=7.6 \mathrm{~Hz}, 1 \mathrm{H}), 1.33(\mathrm{~s}, 12 \mathrm{H}) ;{ }^{13} \mathrm{C} \mathrm{NMR}\left(75 \mathrm{MHz}, \mathrm{CDCl}_{3}\right): 137.52,134.22,133.14,129.54$, $122.48,84.2,24.89$; The carbon which was attached to the boron atom was not detected due to quadrupolar broadening. GC-MS M+ m/z 282.0.<smiles>FC(F)(F)c1cccc(Br)c1</smiles>

2s

4,4,5,5-tetramethyl-2-(3-(trifluoromethyl)phenyl)-1,3,2-dioxaborolane $\quad(2 \mathrm{~s}) .{ }^{11}$ Colorless oil $(17.1 \mathrm{mg}, 63 \%) ;{ }^{1} \mathrm{H}$ NMR $\left(300 \mathrm{MHz}, \mathrm{CDCl}_{3}\right): \delta=8.07(\mathrm{~s}, 1 \mathrm{H}), 7.98(\mathrm{~d}, J=7.4 \mathrm{~Hz}, 1 \mathrm{H}), 7.70(\mathrm{~d}, J$ $=7.8 \mathrm{~Hz}, 1 \mathrm{H}), 7.48(\mathrm{t}, J=7.6 \mathrm{~Hz}, 1 \mathrm{H}), 1.36(\mathrm{~s}, 12 \mathrm{H}) ;{ }^{13} \mathrm{C} \mathrm{NMR}\left(75 \mathrm{MHz}, \mathrm{CDCl}_{3}\right): 138.0,131.4(\mathrm{q}$, $J=3.6 \mathrm{~Hz}), 130.1(\mathrm{q}, J=32.0 \mathrm{~Hz}), 128.0,127.8(\mathrm{q}, J=3.7 \mathrm{~Hz}), 125.7,123.0,84.3,24.8 ;{ }^{19} \mathrm{~F}$ NMR $\left(282 \mathrm{MHz}, \mathrm{CDCl}_{3}\right): \delta=-62.61$. The carbon which was attached to the boron atom was not detected due to quadrupolar broadening. GC-MS $\mathrm{M}^{+} \mathrm{m} / \mathrm{z} 272.1$.<smiles>O=C(O)c1cccc(Br)c1</smiles>

2t

3-(4,4,5,5-tetramethyl-1,3,2-dioxaborolan-2-yl)benzoic acid (2t). ${ }^{14}$ White solid (13.3 mg, 54\%); ${ }^{1} \mathrm{H}$ NMR $\left(300 \mathrm{MHz}, \mathrm{CDCl}_{3}\right): \delta=8.56(\mathrm{~s}, 1 \mathrm{H}), 8.19(\mathrm{~d}, J=9.4 \mathrm{~Hz}, 1 \mathrm{H}), 8.04(\mathrm{~d}, J=7.4 \mathrm{~Hz}, 1 \mathrm{H})$, $7.49(\mathrm{t}, J=7.6 \mathrm{~Hz}, 1 \mathrm{H}), 1.37$ (s, $12 \mathrm{H}) ;{ }^{13} \mathrm{C}$ NMR $\left(75 \mathrm{MHz}, \mathrm{CDCl}_{3}\right): 171.1,140.0,136.6,132.8$, 
$128.7,127.9,84.2,24.9$; The carbon which was attached to the boron atom was not detected due to quadrupolar broadening.<smiles>CCOC(=O)c1cccc(Br)c1</smiles>

2u

ethyl 3-(4,4,5,5-tetramethyl-1,3,2-dioxaborolan-2-yl)benzoate $(\mathbf{2 u}) .^{15}$ White solid (16.8 mg, $61 \%) ;{ }^{1} \mathrm{H}$ NMR (300 MHz, $\left.\mathrm{CDCl}_{3}\right): \delta=8.46(\mathrm{~s}, 1 \mathrm{H}), 8.13(\mathrm{~d}, J=7.8 \mathrm{~Hz}, 1 \mathrm{H}), 7.98(\mathrm{~d}, J=7.3 \mathrm{~Hz}$, 1H), 7.44 (t, $J=7.6 \mathrm{~Hz}, 1 \mathrm{H}), 4.39$ (q, $J=7.1 \mathrm{~Hz}, 2 \mathrm{H}), 1.41(\mathrm{~d}, J=7.1 \mathrm{~Hz}, 3 \mathrm{H}), 1.36(\mathrm{~s}, 12 \mathrm{H}) ;{ }^{13} \mathrm{C}$ NMR (75 MHz, $\mathrm{CDCl}_{3}$ ): 166.8, 139.1, 135.7, 132.3, 130.0, 127.8, 84.1, 61.0, 24.9, 14.4; The carbon which was attached to the boron atom was not detected due to quadrupolar broadening. GC-MS $\mathrm{M}^{+}$ $\mathrm{m} / \mathrm{z} 276.1$.<smiles>Cc1ccccc1Br</smiles>

2v

4,4,5,5-tetramethyl-2-(o-tolyl)-1,3,2-dioxaborolane (2v). ${ }^{5}$ White solid (15.2 mg, 70\%); ${ }^{1} \mathrm{H}$ NMR $\left(300 \mathrm{MHz}, \mathrm{CDCl}_{3}\right): \delta=7.76(\mathrm{~d}, J=8.1 \mathrm{~Hz}, 1 \mathrm{H}), 7.30(\mathrm{td}, J=7.5,1.4 \mathrm{~Hz}, 1 \mathrm{H}), 7.19-7.09(\mathrm{~m}, 2 \mathrm{H})$, $2.54(\mathrm{~s}, 3 \mathrm{H}), 1.33(\mathrm{~s}, 12 \mathrm{H}) ;{ }^{13} \mathrm{C} \mathrm{NMR}\left(75 \mathrm{MHz}, \mathrm{CDCl}_{3}\right): \delta=144.9,136.0,130.9,129.9,124.8,83.5$, 25.0, 22.3; The carbon which was attached to the boron atom was not detected due to quadrupolar broadening. GC-MS M+ $\mathrm{m} / \mathrm{z} 218.1$.<smiles>Oc1ccccc1Br</smiles>

2w

2-(4,4,5,5-tetramethyl-1,3,2-dioxaborolan-2-yl)phenol (2w). ${ }^{14}$ Colorless oil (6.2 mg, $\left.28 \%\right)$; ${ }^{1} \mathrm{H}$ $\operatorname{NMR}\left(300 \mathrm{MHz}, \mathrm{CDCl}_{3}\right): \delta=7.80(\mathrm{~s}, 1 \mathrm{H}), 7.61(\mathrm{~d}, J=7.6,1.9 \mathrm{~Hz}, 1 \mathrm{H}), 7.37(\mathrm{t}, J=8.3,7.3,1.8$ $\mathrm{Hz}, 1 \mathrm{H}), 6.92-6.83(\mathrm{~m}, 2 \mathrm{H}), 1.36$ (s, 12H); $\left.{ }^{13} \mathrm{C} \mathrm{NMR} \mathrm{(75} \mathrm{MHz,} \mathrm{CDCl}_{3}\right): 163.7,135.8,133.9$, 119.6, 115.5, 84.5, 24.8; The carbon which was attached to the boron atom was not detected due to quadrupolar broadening. GC-MS M+ $\mathrm{m} / \mathrm{z} 220.1$.<smiles>COc1ccccc1Br</smiles>

$2 x$

2-(2-methoxyphenyl)-4,4,5,5-tetramethyl-1,3,2-dioxaborolane (2x). ${ }^{16}$ Colorless oil (4.7 $\mathrm{mg}$, $20 \%) ;{ }^{1} \mathrm{H}$ NMR $\left(300 \mathrm{MHz}, \mathrm{CDCl}_{3}\right): \delta=7.68(\mathrm{~d}, J=7.3,1.9 \mathrm{~Hz}, 1 \mathrm{H}), 7.39(\mathrm{t}, J=8.3,7.3,1.9 \mathrm{~Hz}$, $1 \mathrm{H}), 6.94(\mathrm{t}, J=7.3,0.9 \mathrm{~Hz}, 1 \mathrm{H}), 6.85(\mathrm{~d}, J=8.4,0.9 \mathrm{~Hz}, 1 \mathrm{H}), 3.83(\mathrm{~s}, 3 \mathrm{H}), 1.35(\mathrm{~s}, 12 \mathrm{H}) ;{ }^{13} \mathrm{C} \mathrm{NMR}$ 
(75 MHz, $\left.\mathrm{CDCl}_{3}\right): 164.1,136.7,132.5,120.2,110.4,83.5,55.8,24.8$; The carbon which was attached to the boron atom was not detected due to quadrupolar broadening. GC-MS M+ $\mathrm{m} / \mathrm{z}$ 234.1.<smiles>Fc1ccccc1Br</smiles>

$2 y$

2-(2-fluorophenyl)-4,4,5,5-tetramethyl-1,3,2-dioxaborolane (2y). ${ }^{11}$ Colorless oil (14.2 mg, 64\%); ${ }^{1} \mathrm{H}$ NMR $\left(300 \mathrm{MHz}, \mathrm{CDCl}_{3}\right): \delta=7.74(\mathrm{t}, J=6.7 \mathrm{~Hz}, 1 \mathrm{H}), 7.51-7.43(\mathrm{~m}, 1 \mathrm{H}), 7.13(\mathrm{t}, J=7.4 \mathrm{~Hz}$, 1H), 7.03 (t, $J=8.6 \mathrm{~Hz}, 1 \mathrm{H}), 1.37$ (s, 12H); ${ }^{13} \mathrm{C} \mathrm{NMR}\left(75 \mathrm{MHz}, \mathrm{CDCl}_{3}\right): 167.2(\mathrm{~d}, J=250.9 \mathrm{~Hz})$, 136.9 (d, $J=8.0 \mathrm{~Hz}), 133.3$ (d, $J=8.8 \mathrm{~Hz}), 123.6$ (d, $J=3.2 \mathrm{~Hz}), 115.3$ (d, $J=23.9 \mathrm{~Hz}), 83.9,24.8$; ${ }^{19} \mathrm{~F} \mathrm{NMR}\left(282 \mathrm{MHz}, \mathrm{CDCl}_{3}\right): \delta=-102.58$. The carbon which was attached to the boron atom was not detected due to quadrupolar broadening. GC-MS M+ $\mathrm{m} / \mathrm{z} 222.1$.<smiles>Clc1ccccc1Br</smiles>

$2 z$

2-(2-chlorophenyl)-4,4,5,5-tetramethyl-1,3,2-dioxaborolane (2z). ${ }^{10}$ Colorless oil ( $\left.8.0 \mathrm{mg}, 34 \%\right)$; ${ }^{1} \mathrm{H}$ NMR (300 MHz, $\left.\mathrm{CDCl}_{3}\right): \delta=7.68(\mathrm{~d}, J=7.7 \mathrm{~Hz}, 1 \mathrm{H}), 7.42-7.33(\mathrm{~m}, 2 \mathrm{H}), 7.2$ (ddd, $J=10.4$, 5.6, $2.4 \mathrm{~Hz}, 1 \mathrm{H}), 1.37$ (s, 12H); ${ }^{13} \mathrm{C} \mathrm{NMR}\left(75 \mathrm{MHz}, \mathrm{CDCl}_{3}\right)$ : 139.6, 136.4, 131.9, 129.4, 125.8, 84.2, 24.8; The carbon which was attached to the boron atom was not detected due to quadrupolar broadening. GC-MS M+ m/z 238.1.<smiles>COc1ccc(C)cc1</smiles>

4

(4-methoxyphenyl)(methyl)sulfane (4). Yellow oil (9.9 mg, 64\%); ${ }^{1} \mathrm{H} \mathrm{NMR}\left(400 \mathrm{MHz}, \mathrm{CDCl}_{3}\right) \delta$ $=7.25(\mathrm{~d}, J=8.9 \mathrm{~Hz}, 2 \mathrm{H}), 6.83(\mathrm{~d}, J=8.9 \mathrm{~Hz}, 2 \mathrm{H}), 3.76(\mathrm{~s}, 3 \mathrm{H}), 2.42(\mathrm{~s}, 3 \mathrm{H}) .{ }^{13} \mathrm{C} \mathrm{NMR}(101 \mathrm{MHz}$, $\left.\mathrm{CDCl}_{3}\right) \delta=158.2,130.2,128.8,114.7,55.4,18.1$.

\section{References}

1. Minami, H.; Otsuka, S.; Nogi, K.; Yorimitsu, H. ACS Catal. 2018, 8, 579.

2. Jiri, S.; Gary D, A.; Lanny S, L. J. Am. Chem. Soc. 1997, 119, 12376.

3. Zhang, B.-X.; Li, T.-T.; Kang, Y.-Q. Res. Chem. Intermed. 2017, 43, 6617.

4. Miyatake K.; Yamamoto K.; Endo, K.; Tsuchida, E. J. Org. Chem. 1998, 63, 7522.

5. Chen, K.; Zhang, S.; He, P.; Li, P.-F. Chem. Sci. 2016, 7, 3676.

6. Lv, Y.-H.; Sun, K.; Pu, W.-Y.; Mao, S.-K.; Li, G.; Niu, J.-J.; Chen, Q.; Wang, T.-T. RSC Adv. 2016, 6, 93486.

7. Candish, L.; Teders, M.; Glorius, F. J. Am. Chem. Soc. 2017, 139, 7440.

8. Uetake, Y.; Niwa, T.; Hosoya, T. Org. Lett. 2016, 18, 2758. 
9. Nagashima, Y.; Takita, R.; Yoshida, K.; Hirano, K.; Uchiyama, M. J. Am. Chem. Soc. 2013, 135, 18730 .

10. Partridge, B. M.; Thomas, S. P.; Aggarwal, V. K. Tetrahedron 2011, 67, 10082.

11. Zhu, W.; Ma, D.-W. Org. Lett. 2006, 8, 261.

12. Bruce, J, I.; Chambron, J.; Koelle, P.; Sauvage, J, P. J. Chem. Soc., Perkin Trans. 1 2002, 1226.

13. Jiang, Q.; Ryan, M.; Zhichkin, P. J. Org. Chem. 2007, 72, 6618.

14. Hinkes, S. P. A.; Klein, C. D. P. Org. Lett. 2019, 21, 3048.

15. Tobisu, M.; Nakamura, R.; Kita, Y.; Chatani, N. J. Am. Chem. Soc. 2009, 131, 3174.

16. Billingsley, K. L.; Buchwald, S. L. J. Org. Chem. 2008, 73, 5589. 
VI. ${ }^{1} \mathrm{H}$ NMR, ${ }^{13} \mathrm{C}$ NMR and ${ }^{19} \mathrm{~F}$ NMR Spectra
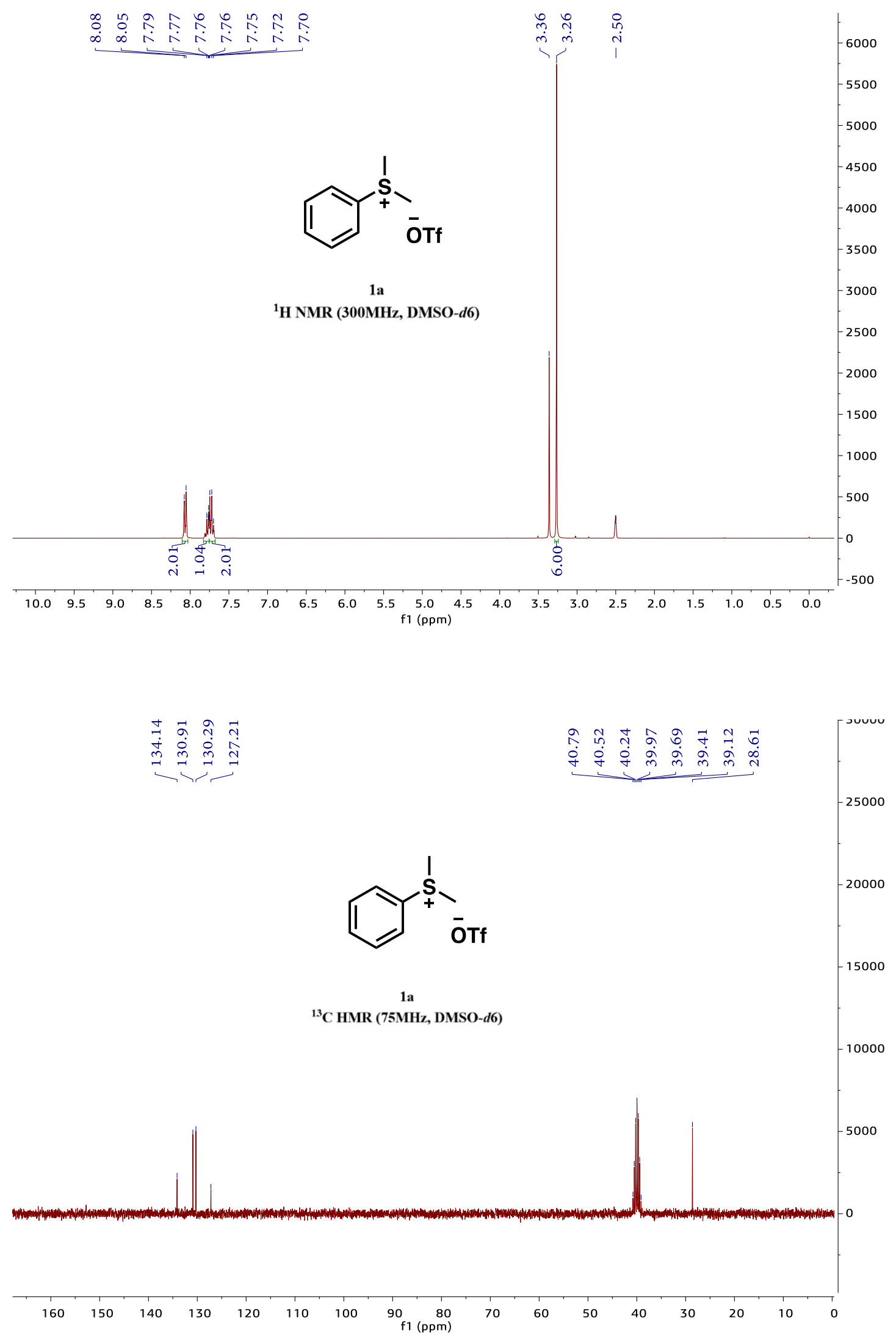


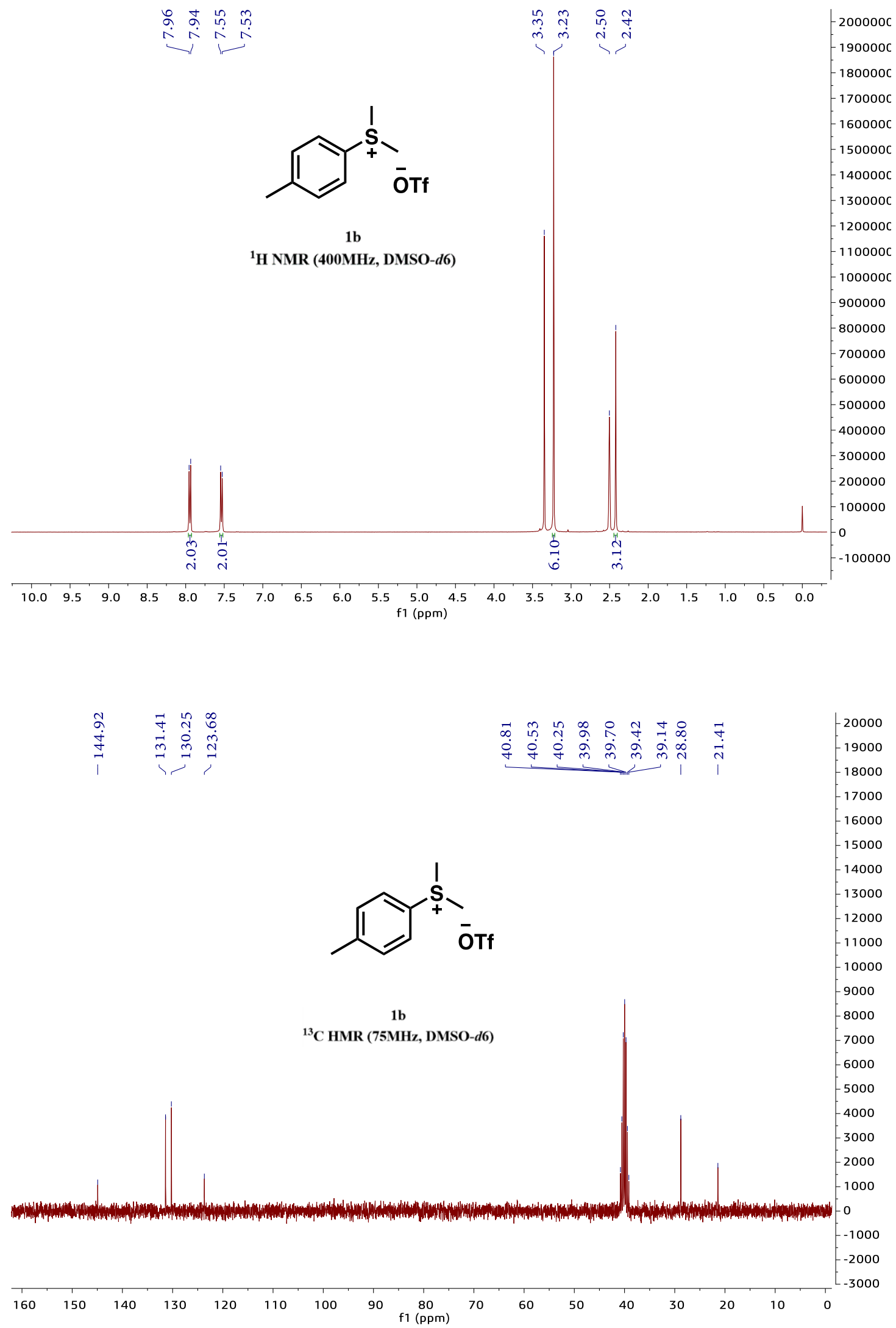




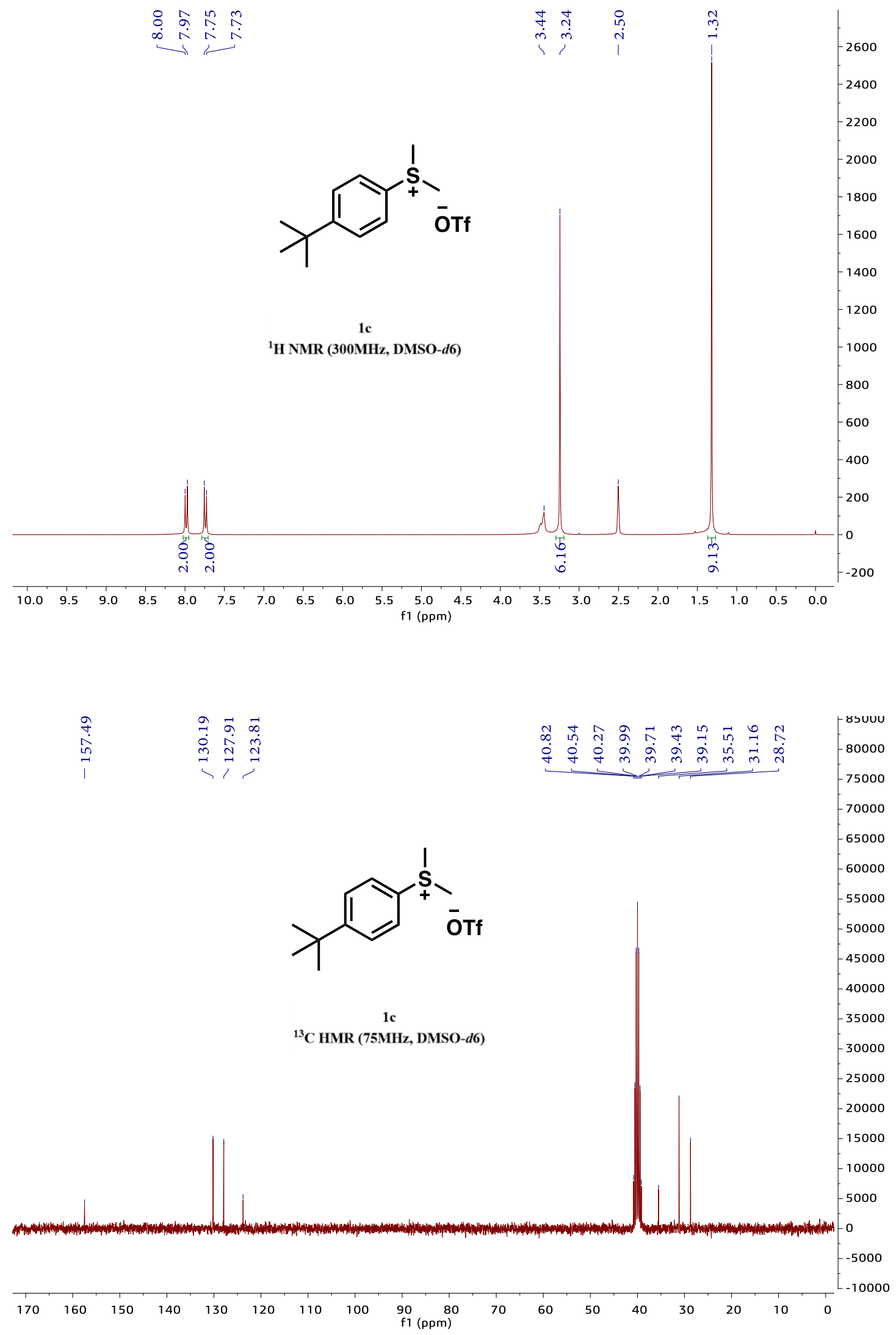




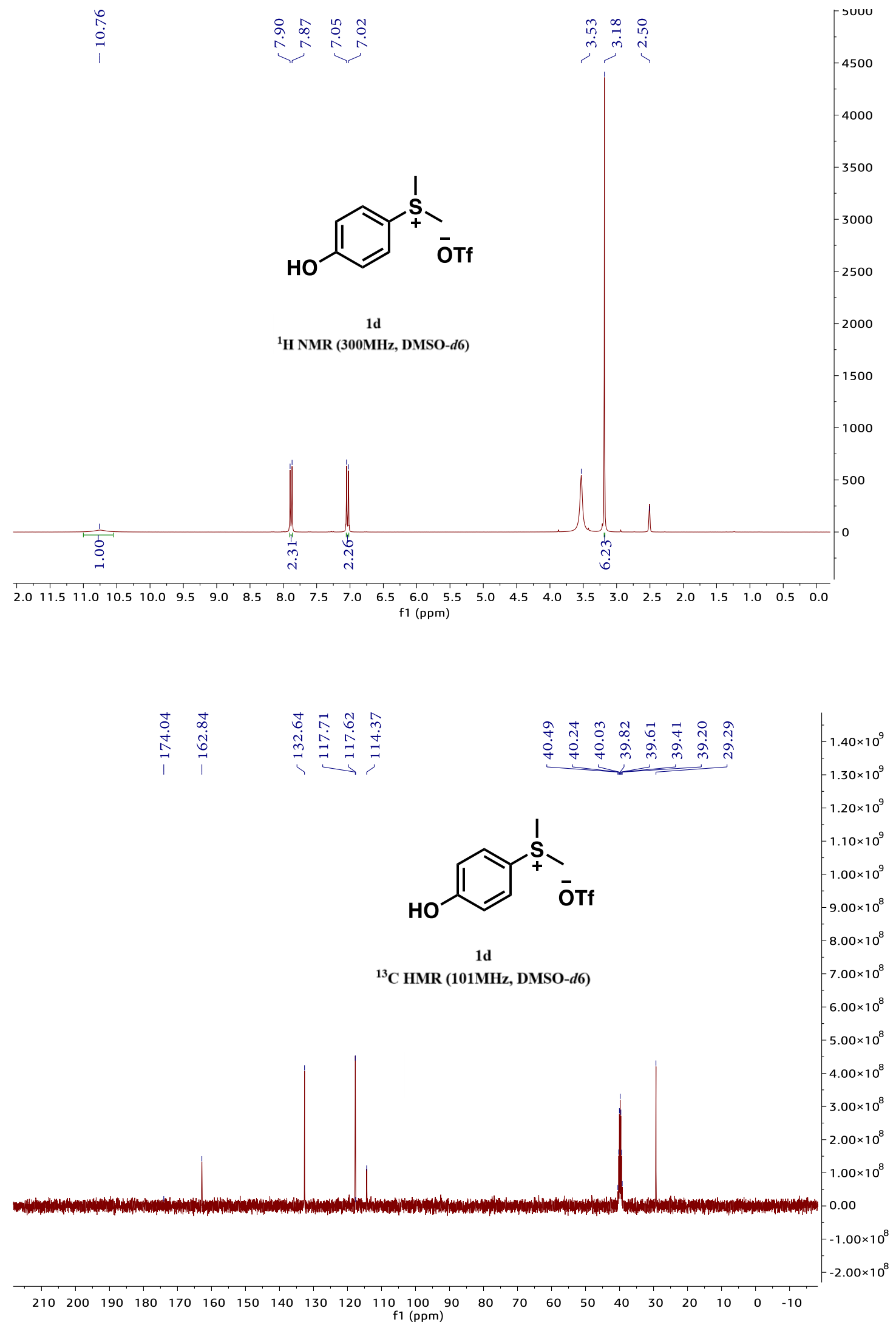




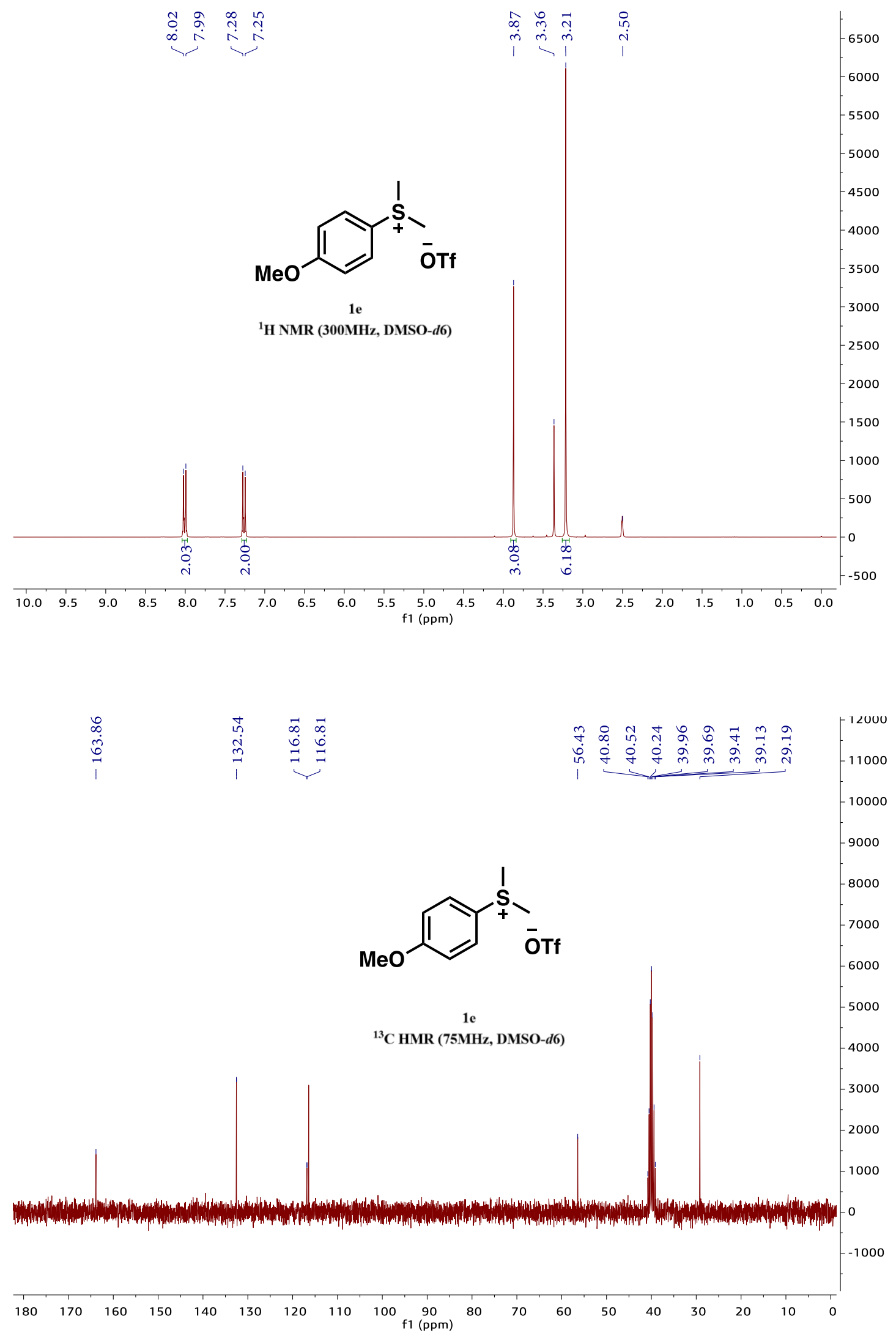



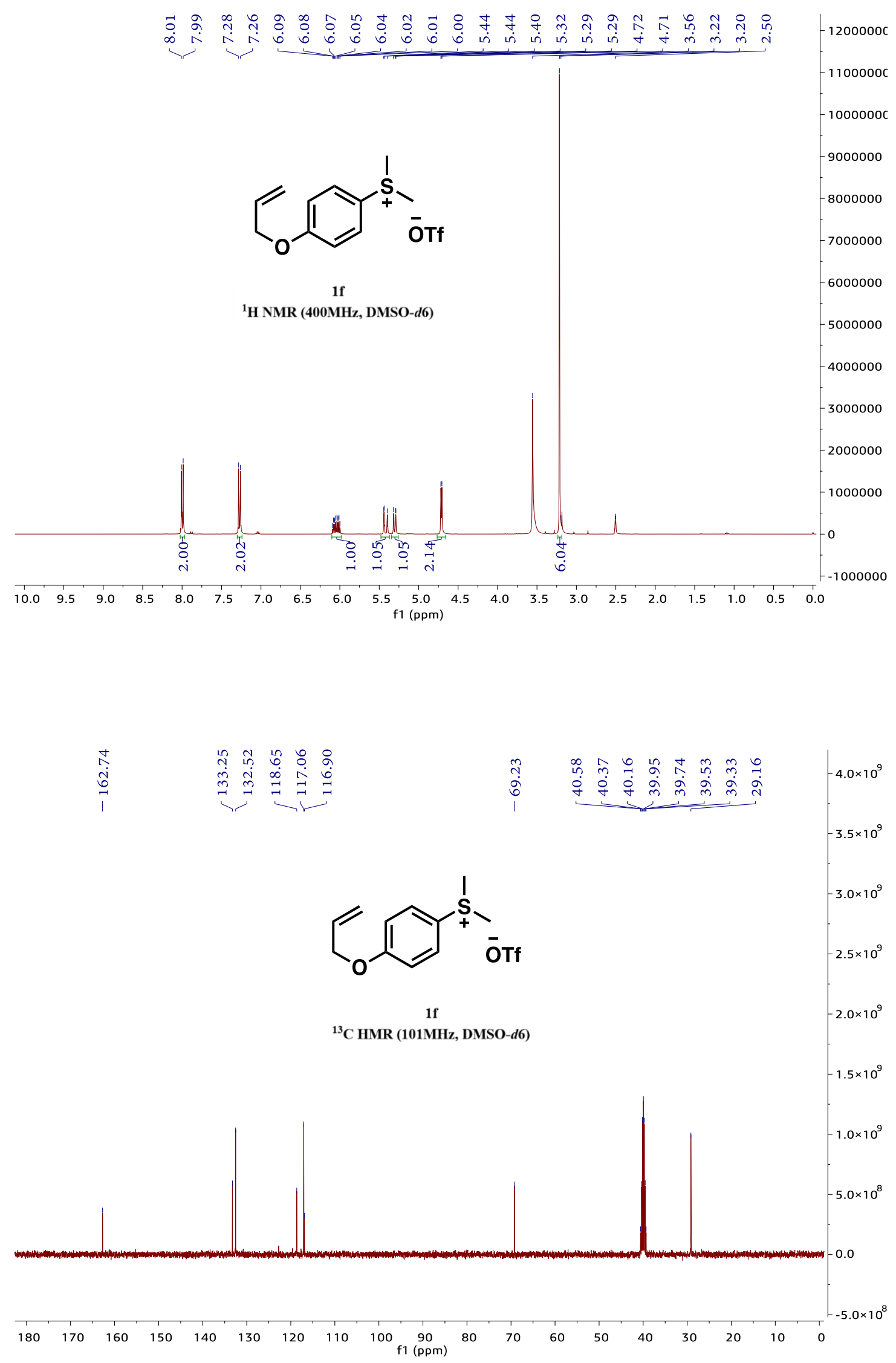


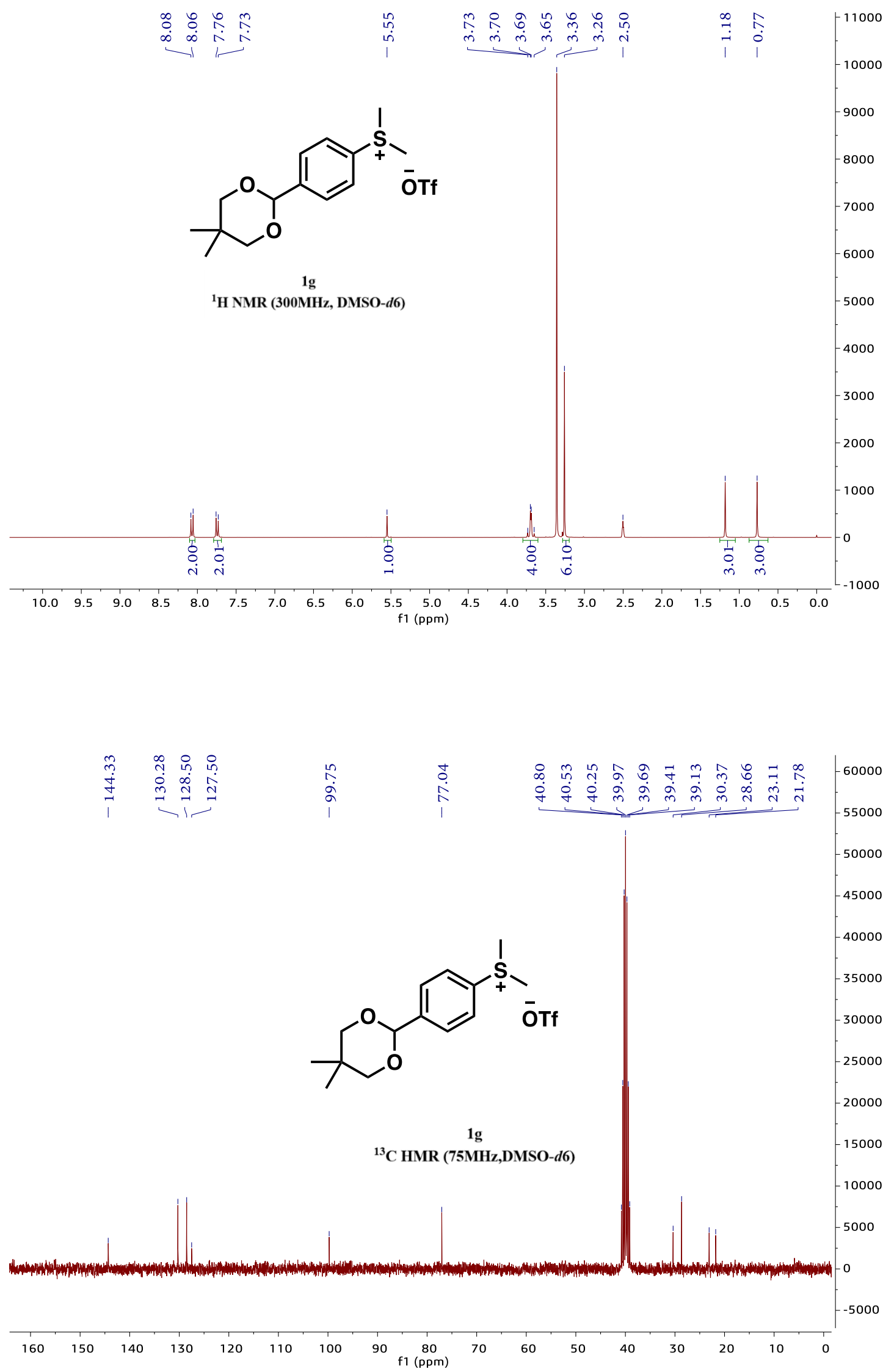




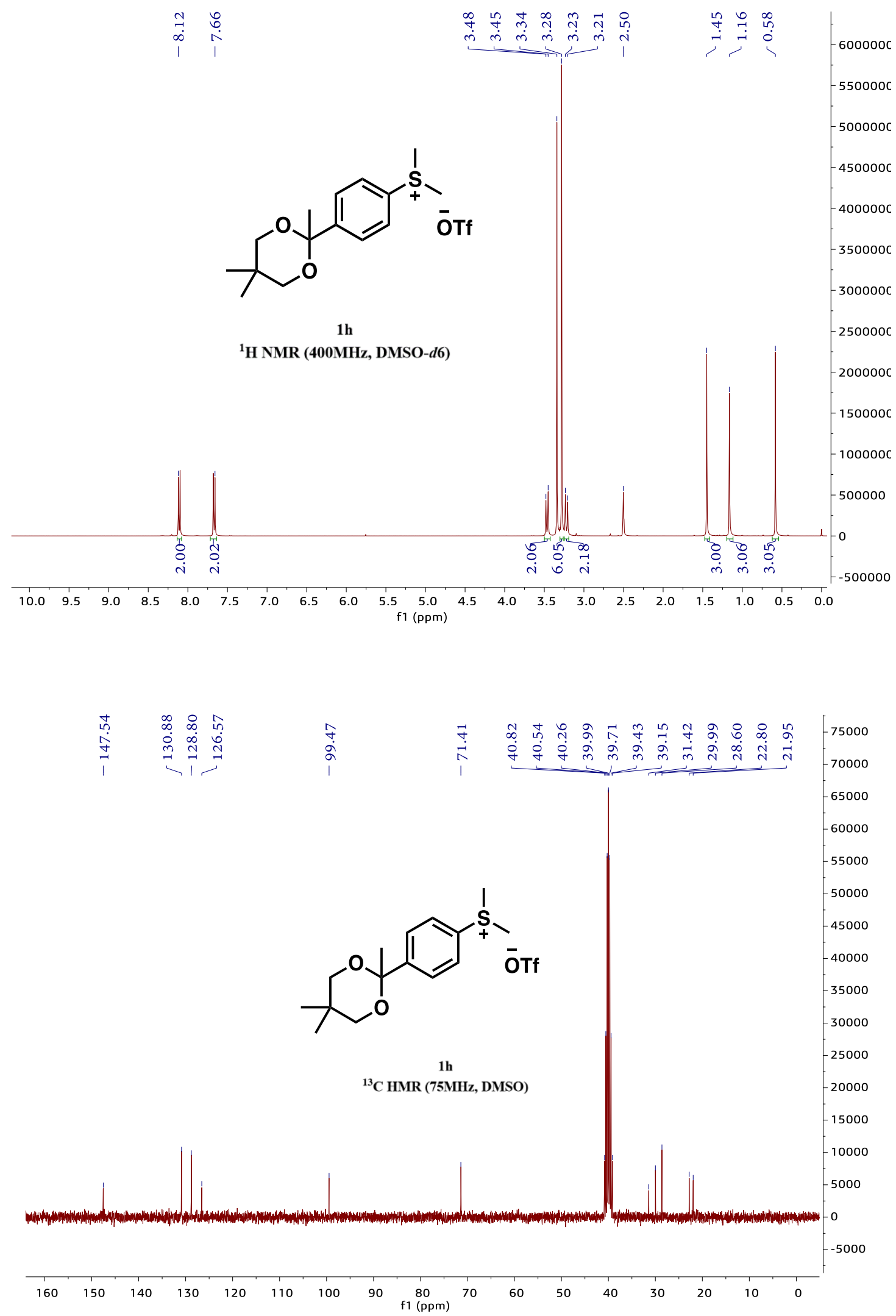




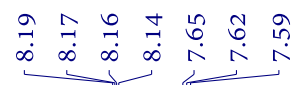

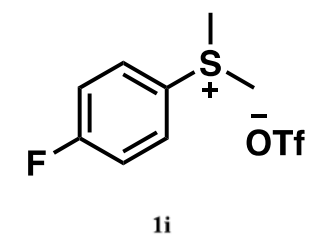

$\begin{array}{lll}\circ & 0 & \text { nे } \\ \dot{r} & \stackrel{n}{i} & \text { i }\end{array}$

5000

500

4000

3500

3000

$-2500$

${ }^{1} \mathrm{H}$ NMR (300MHz, DMSO-d6)

2000

$-1500$

1000

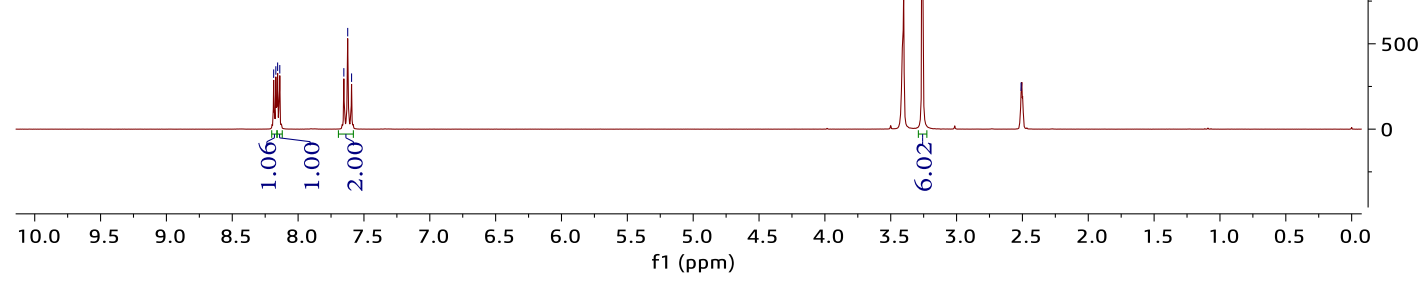

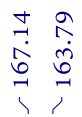<smiles>C[S+](C)c1ccc(F)cc1</smiles>

1i

${ }^{13}$ C HMR (75MHz,DMSO-d6)

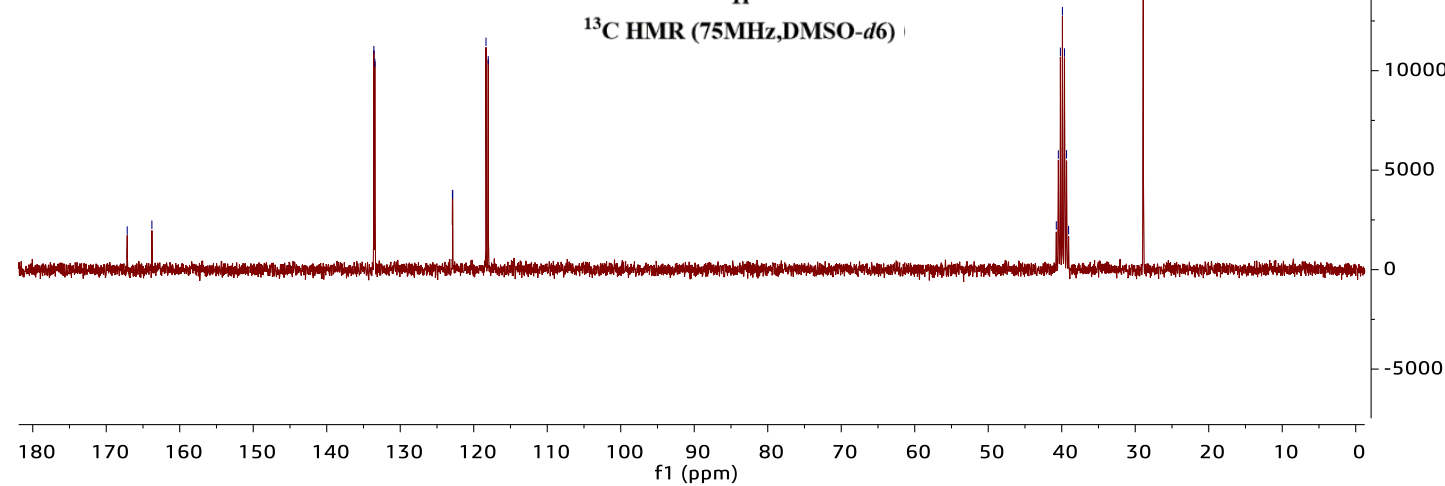



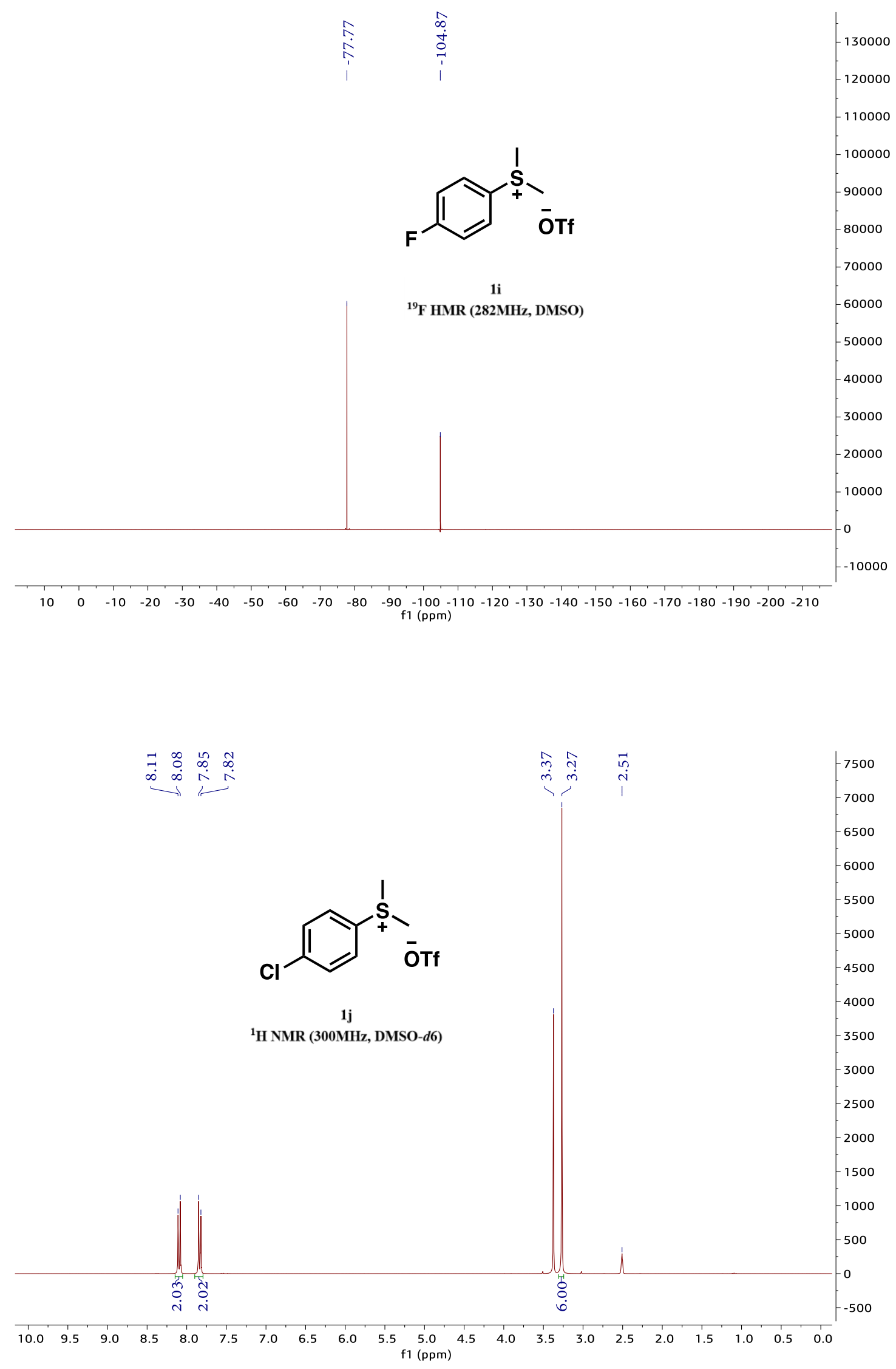

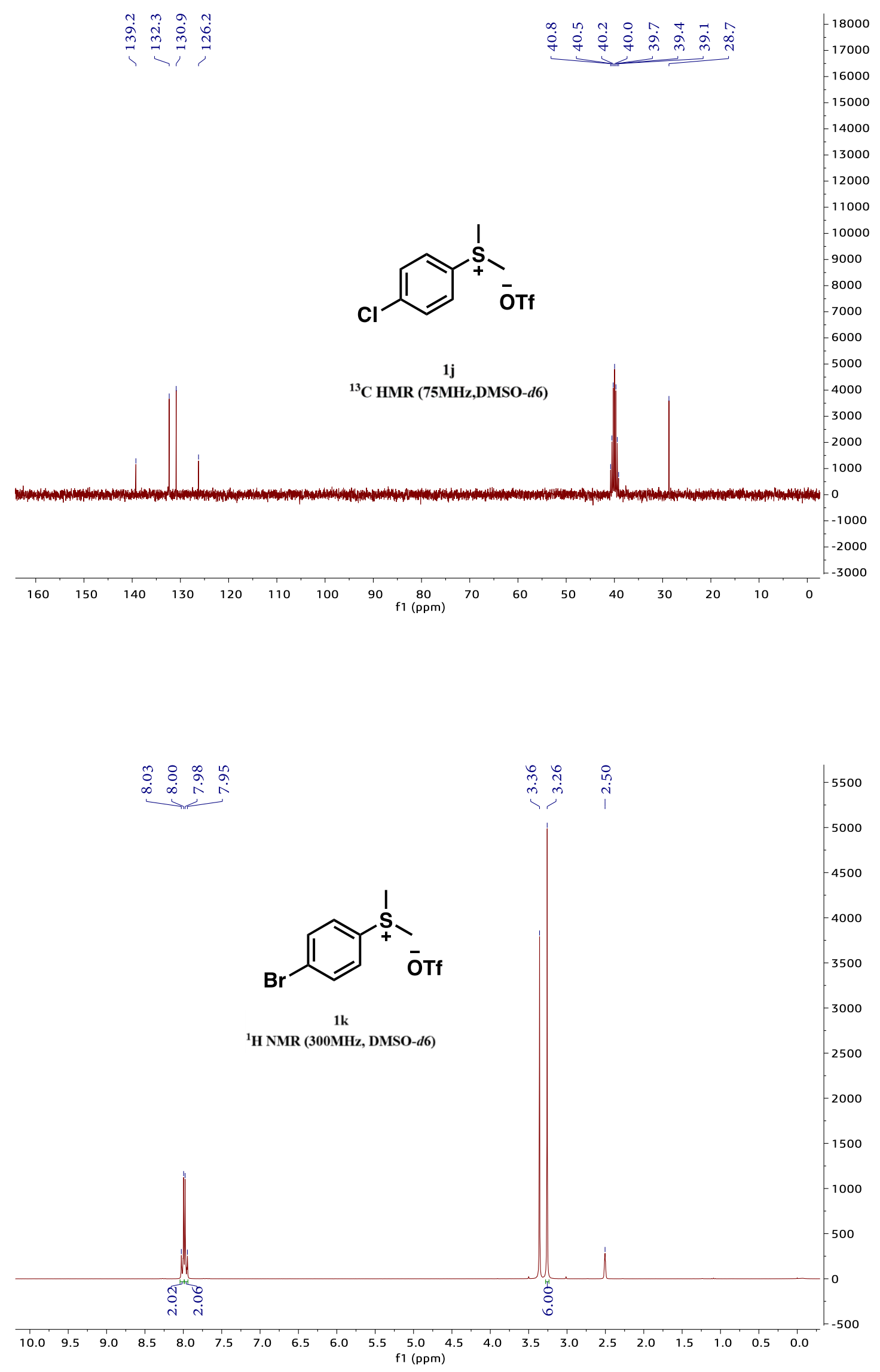

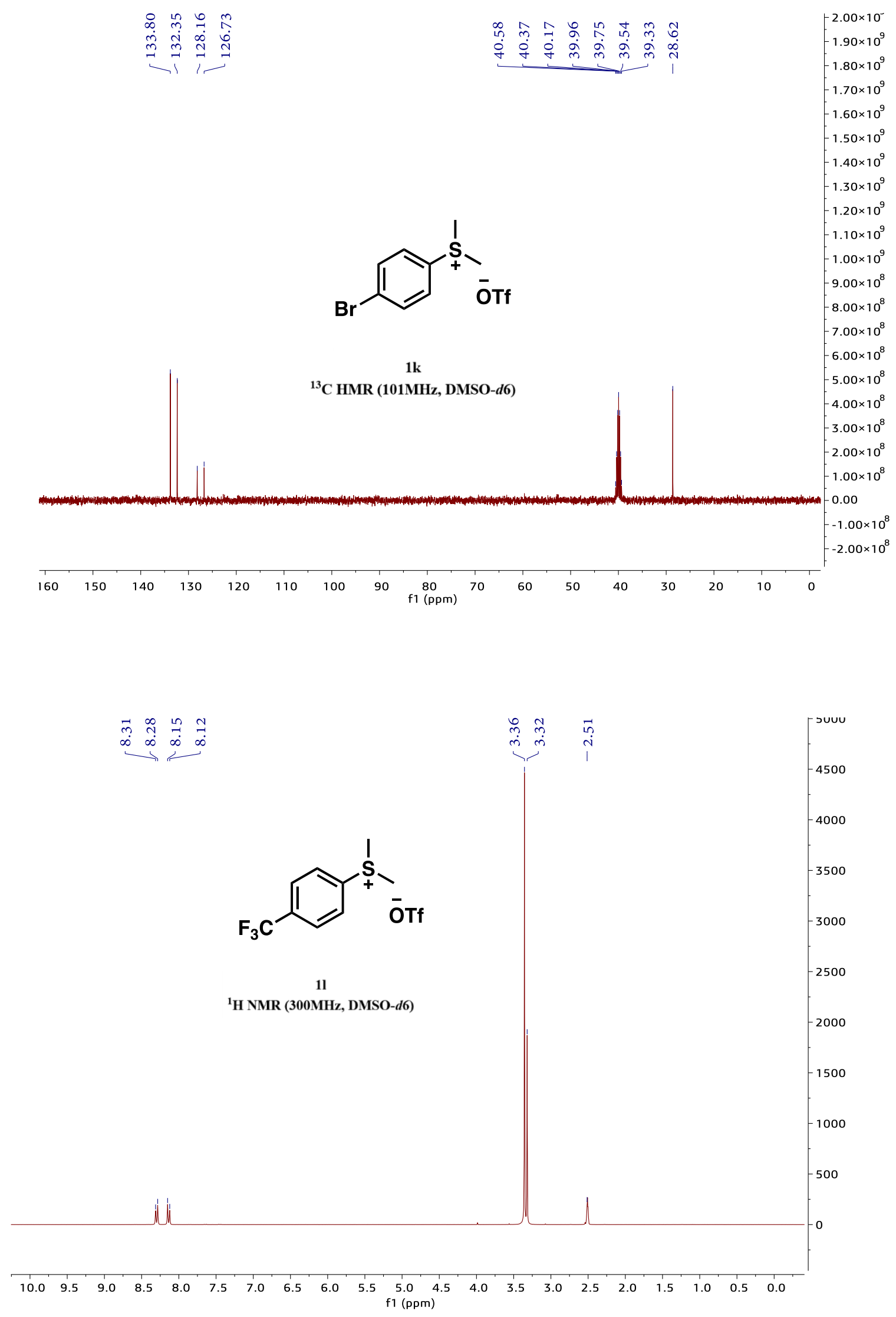

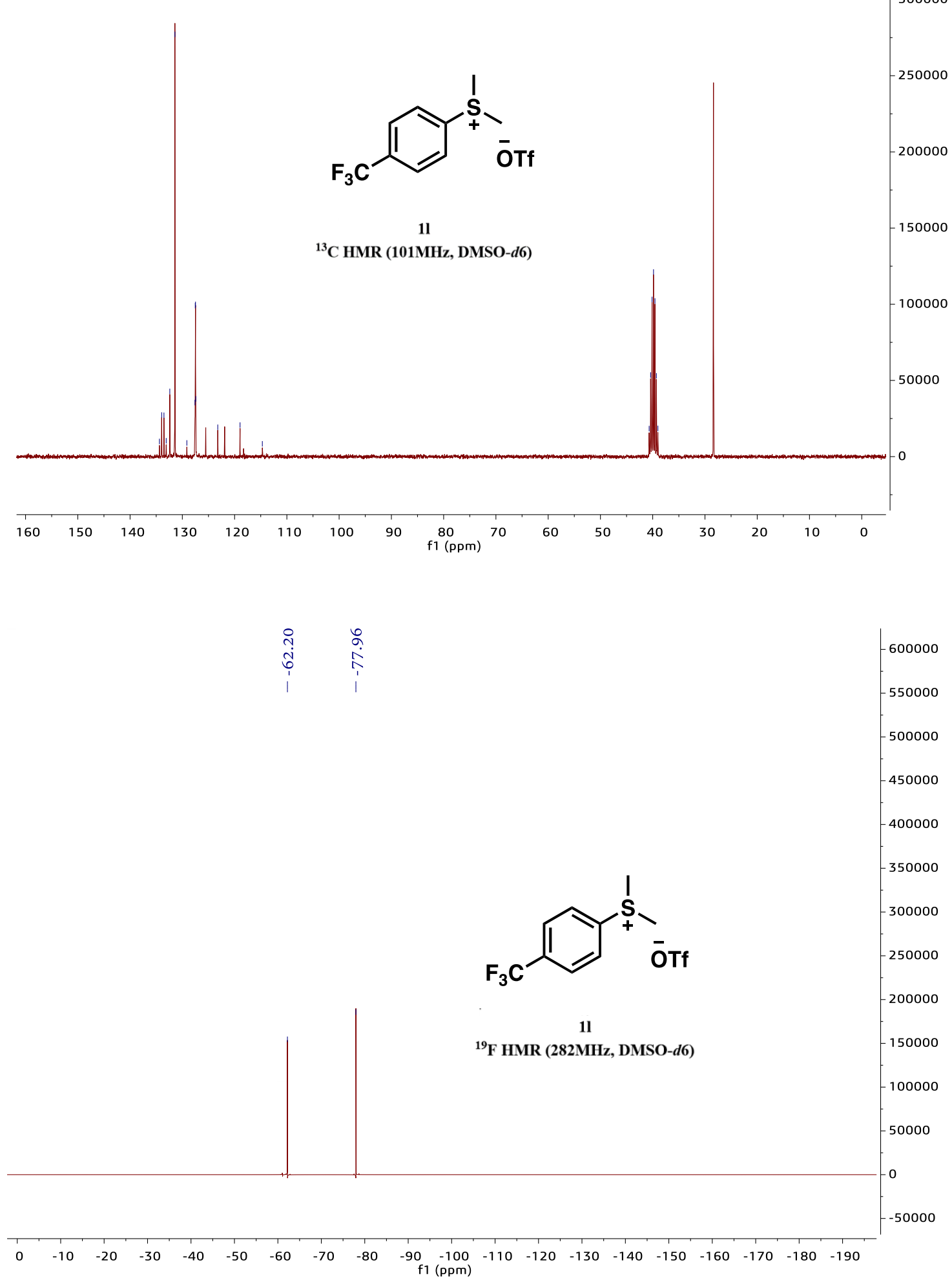

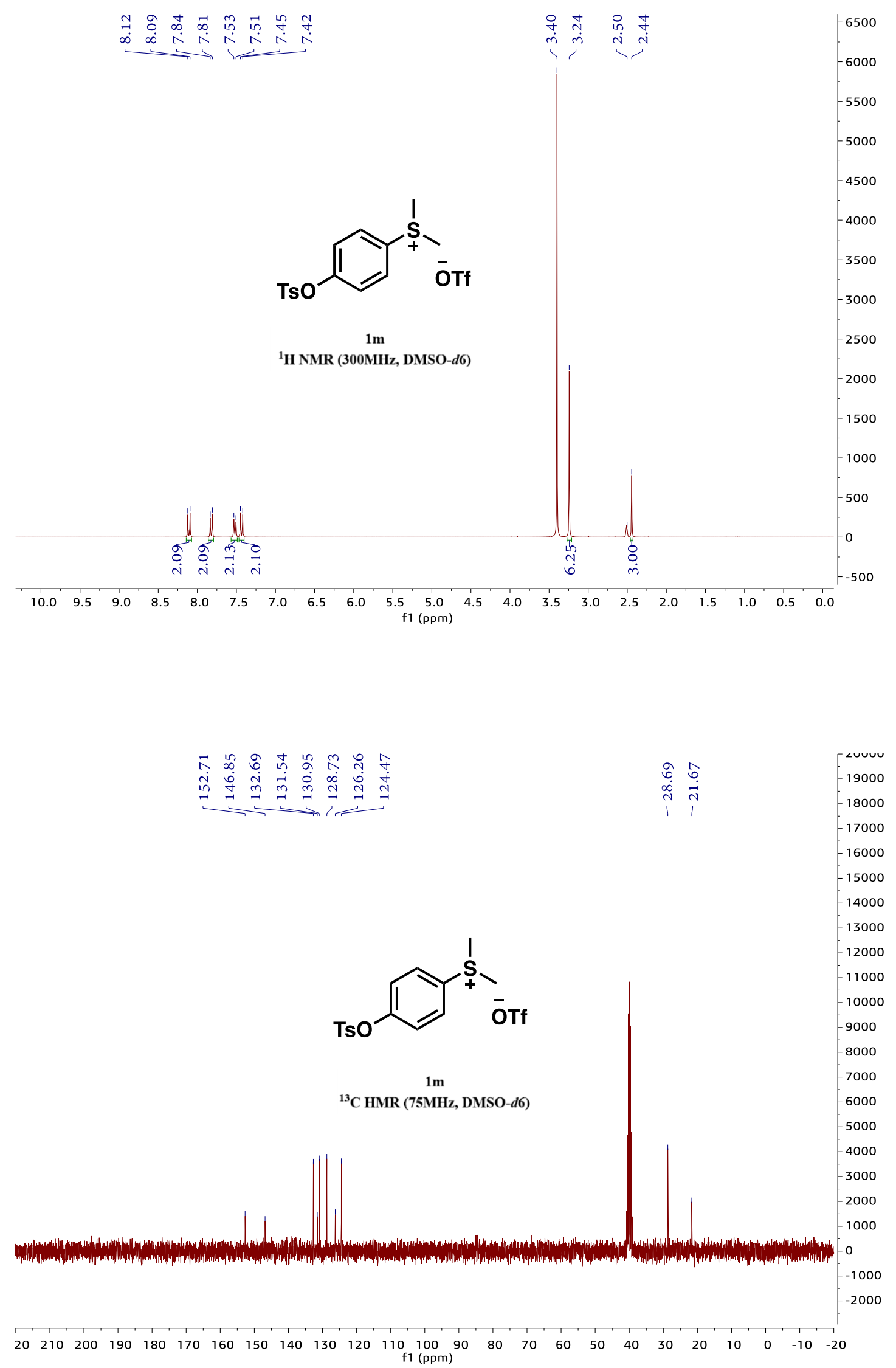

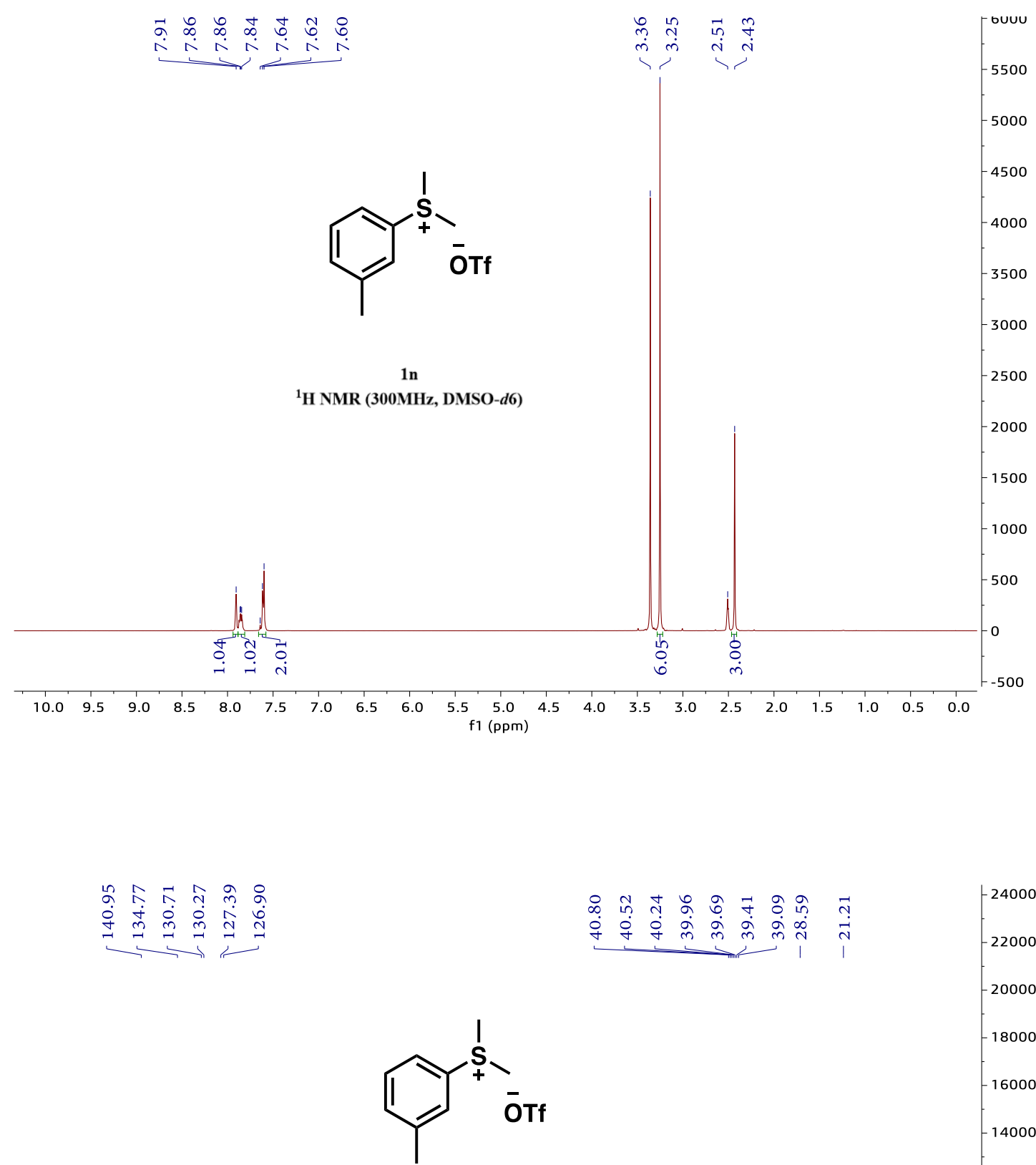

$1 \mathrm{n}$

${ }^{13} \mathrm{C}$ HMR (75MHz, DMSO-d6)

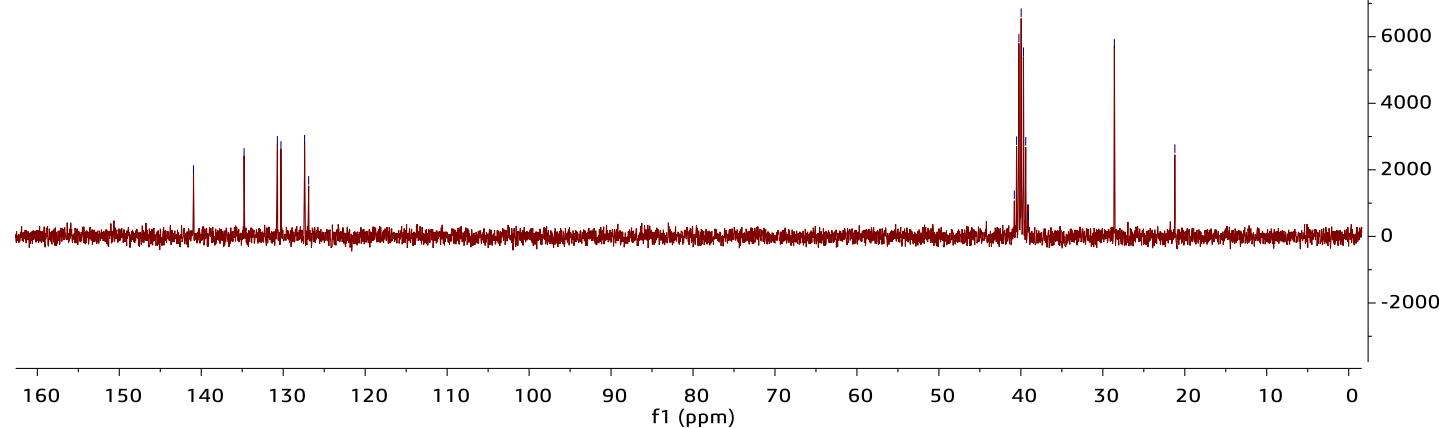



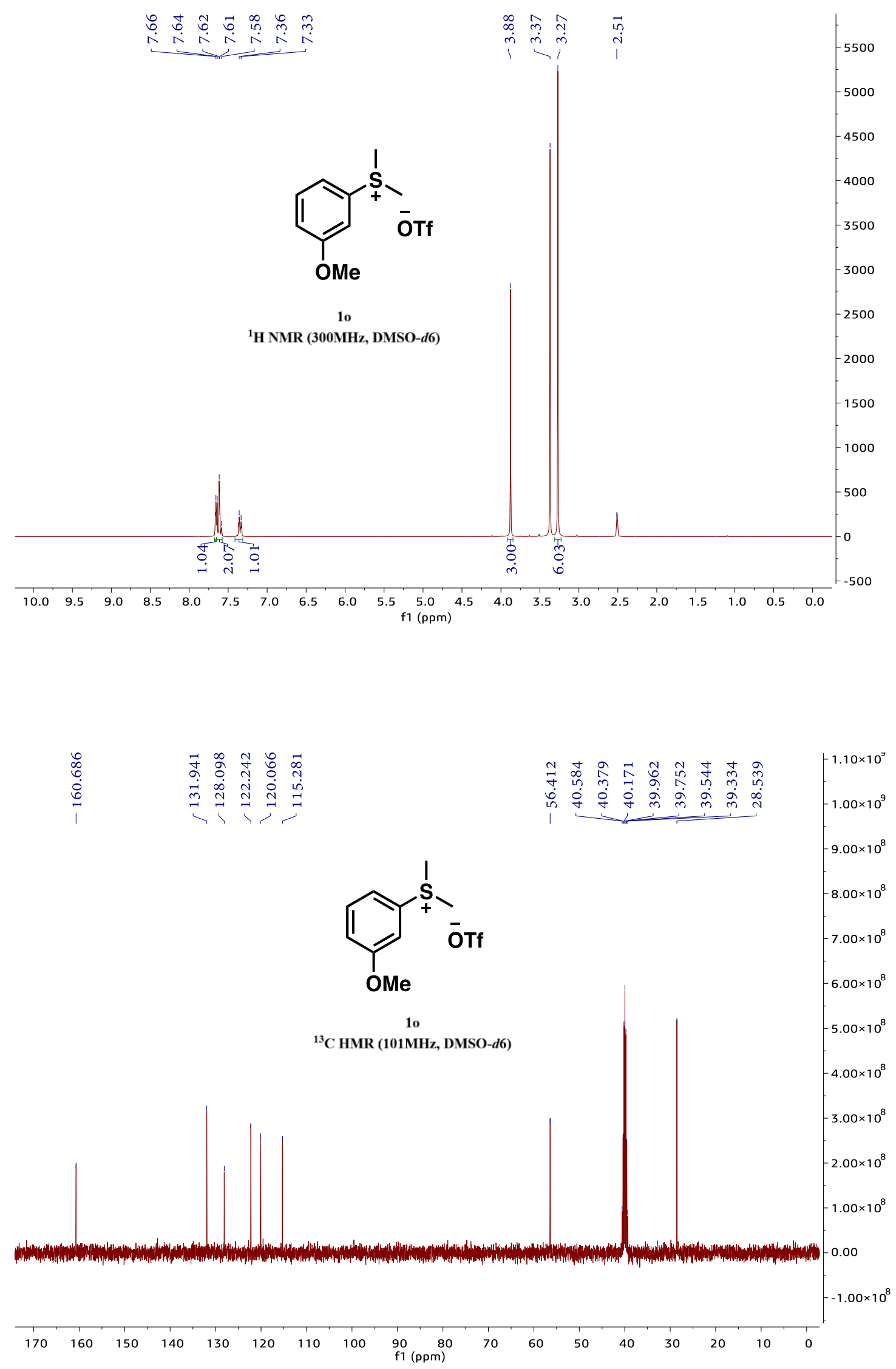


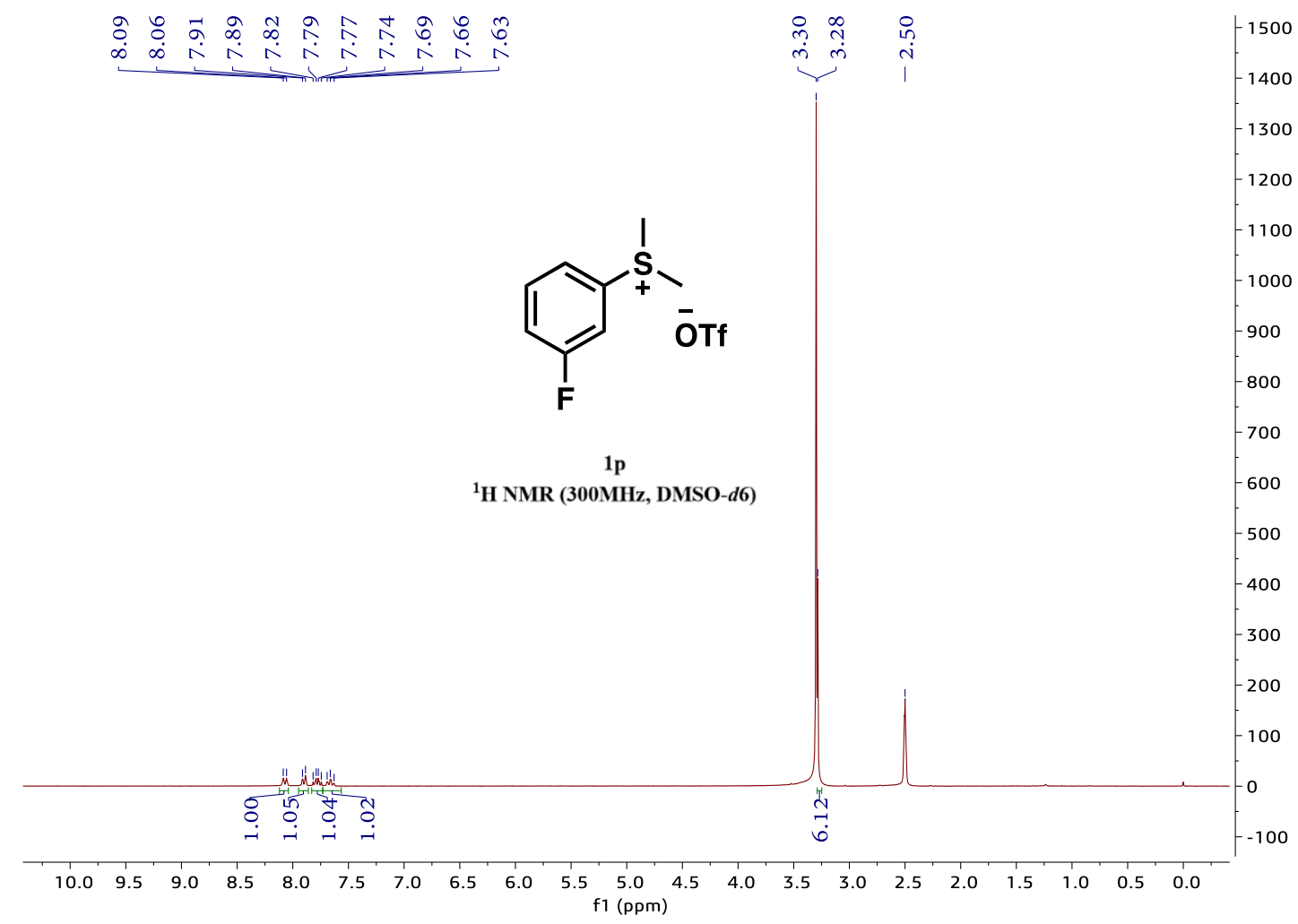

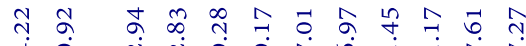

苑

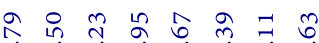

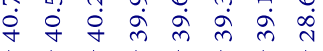

$\angle 8000$

26000

24000

$-22000$<smiles>C[S+](C)c1cccc(F)c1</smiles>

$1 p$

${ }^{13} \mathrm{C}$ HMR (75MHz, DMSO-d6)

$-20000$

18000

16000

$-14000$

12000

10000

$-8000$

$-6000$

$-4000$

$-2000$

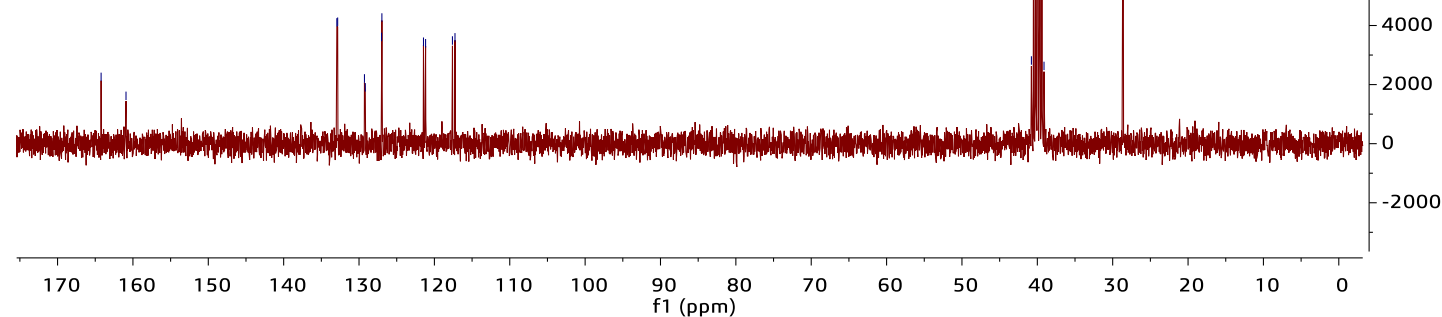



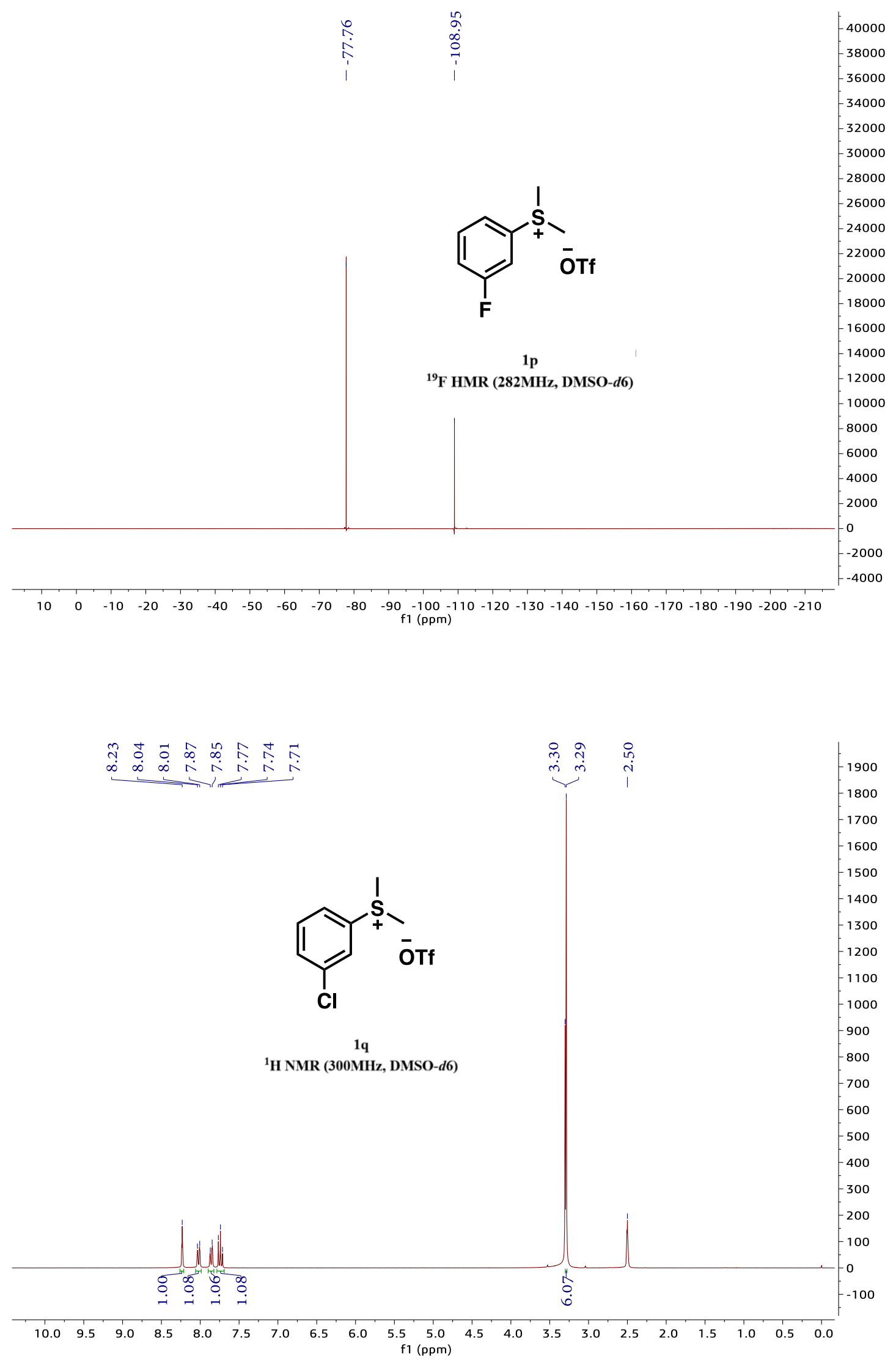

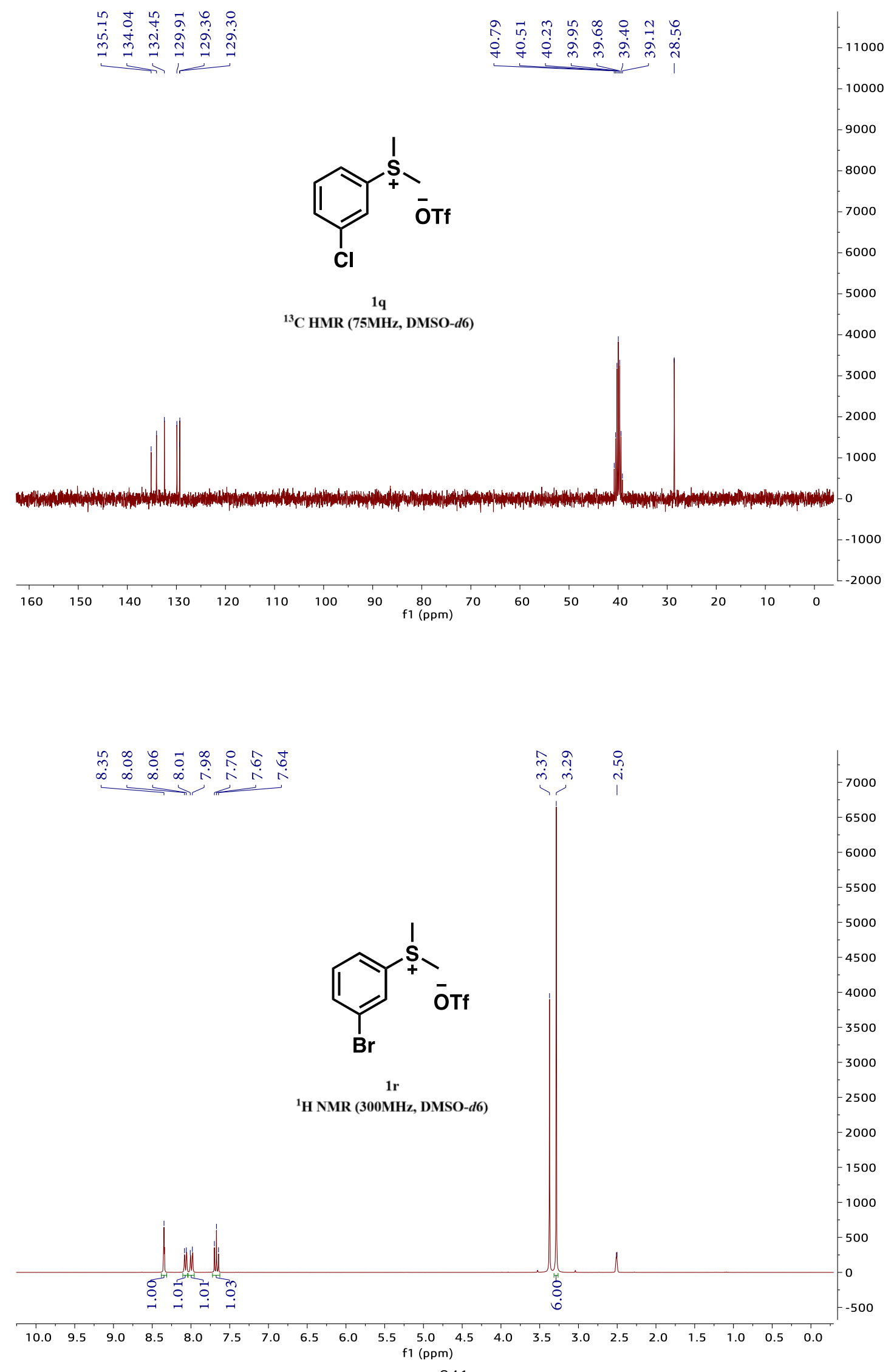

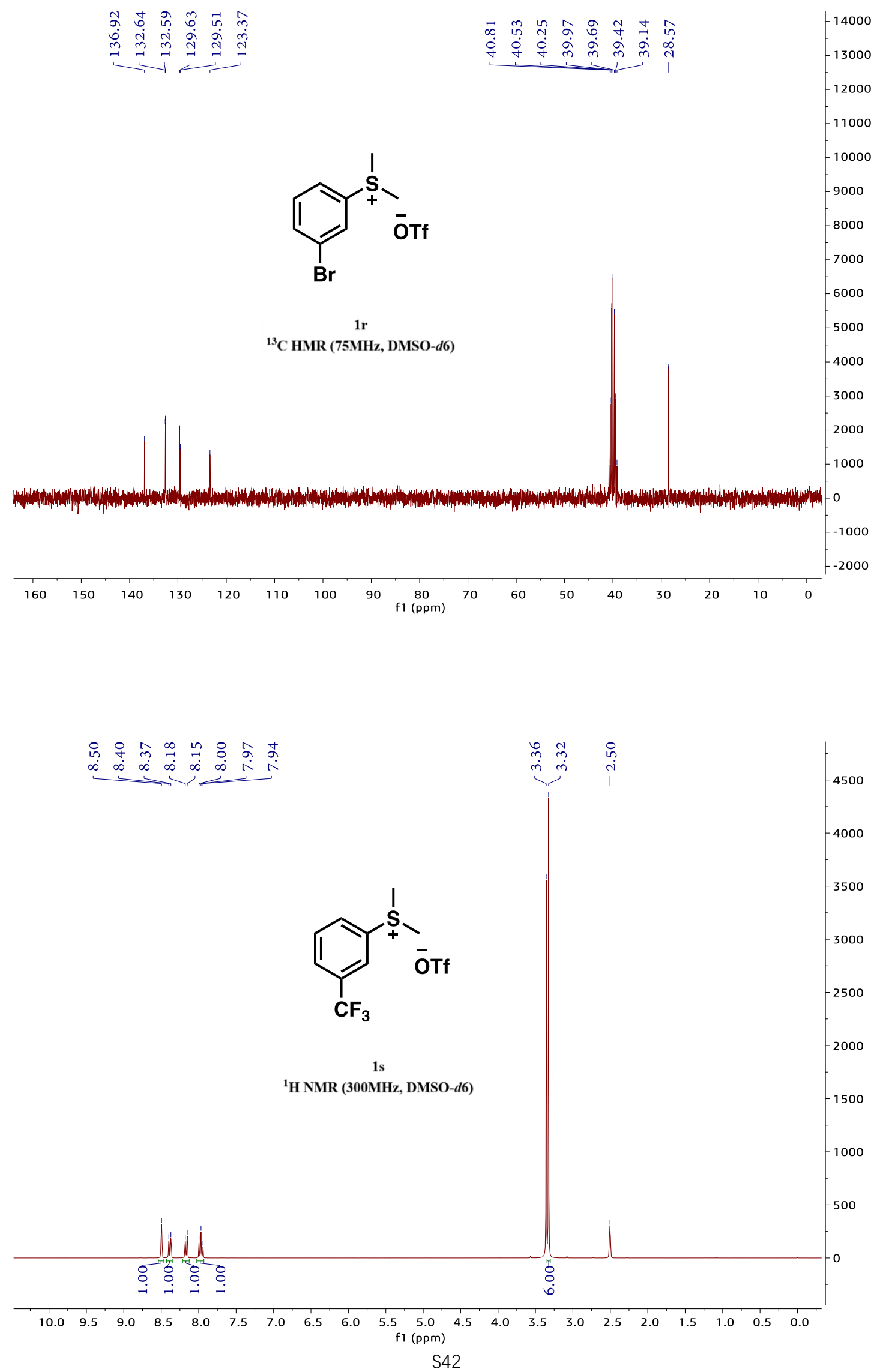
<smiles></smiles>

$1 \mathrm{~s}$

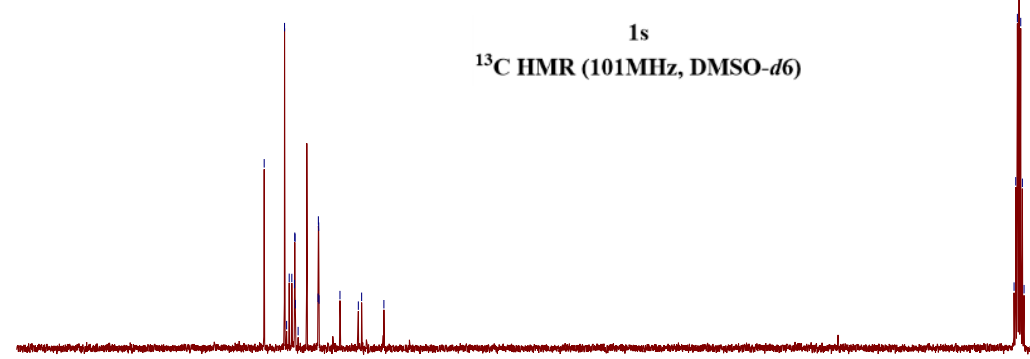

$-170000$

$-160000$

$-150000$

$-140000$

$-130000$

$-120000$

$-110000$

$-100000$

90000

$-80000$

$-70000$

$-60000$

50000

$-40000$

30000

20000

10000

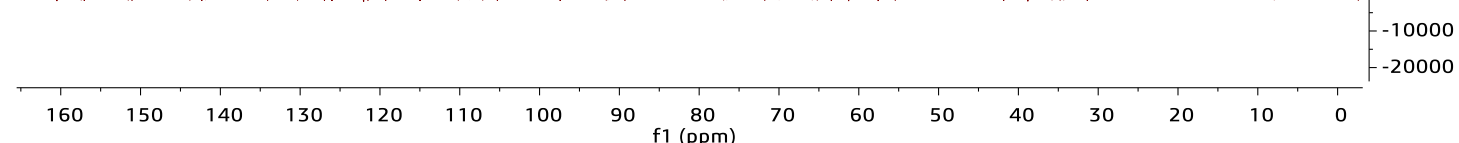

$\begin{array}{ll}\infty & -1 \\ 0 & 0 \\ \dot{0} & \infty \\ 1 & 1 \\ 1 & 1\end{array}$

700000

650000

600000

$-550000$<smiles>CI(C)c1cccc(C(F)(F)F)c1</smiles>

500000

$-450000$

400000

$-350000$

$-300000$

$1 s$

$-250000$

${ }^{19}$ F HMR (282MHz, DMSO-d6)

200000

150000

100000

$-50000$ 


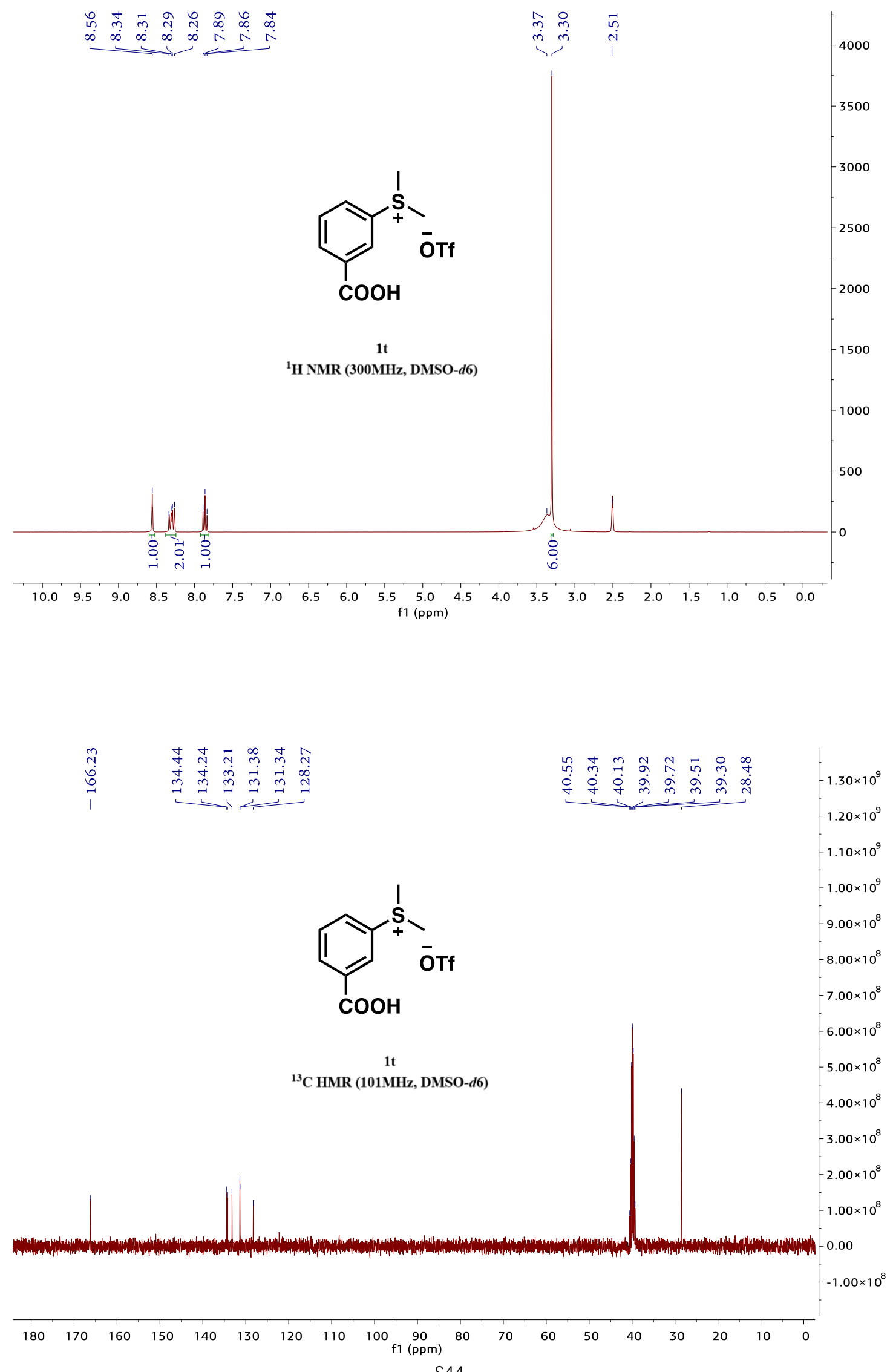



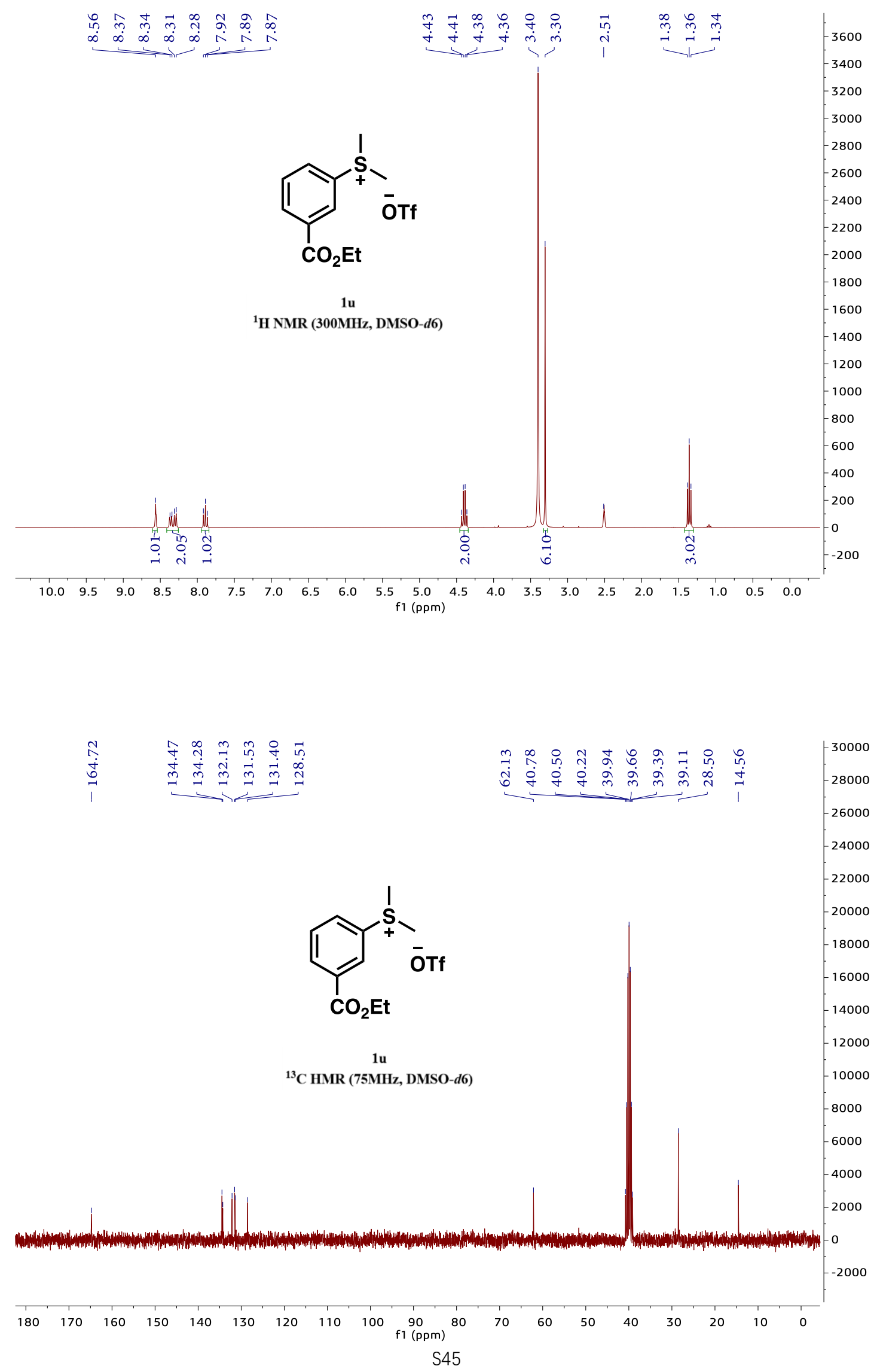

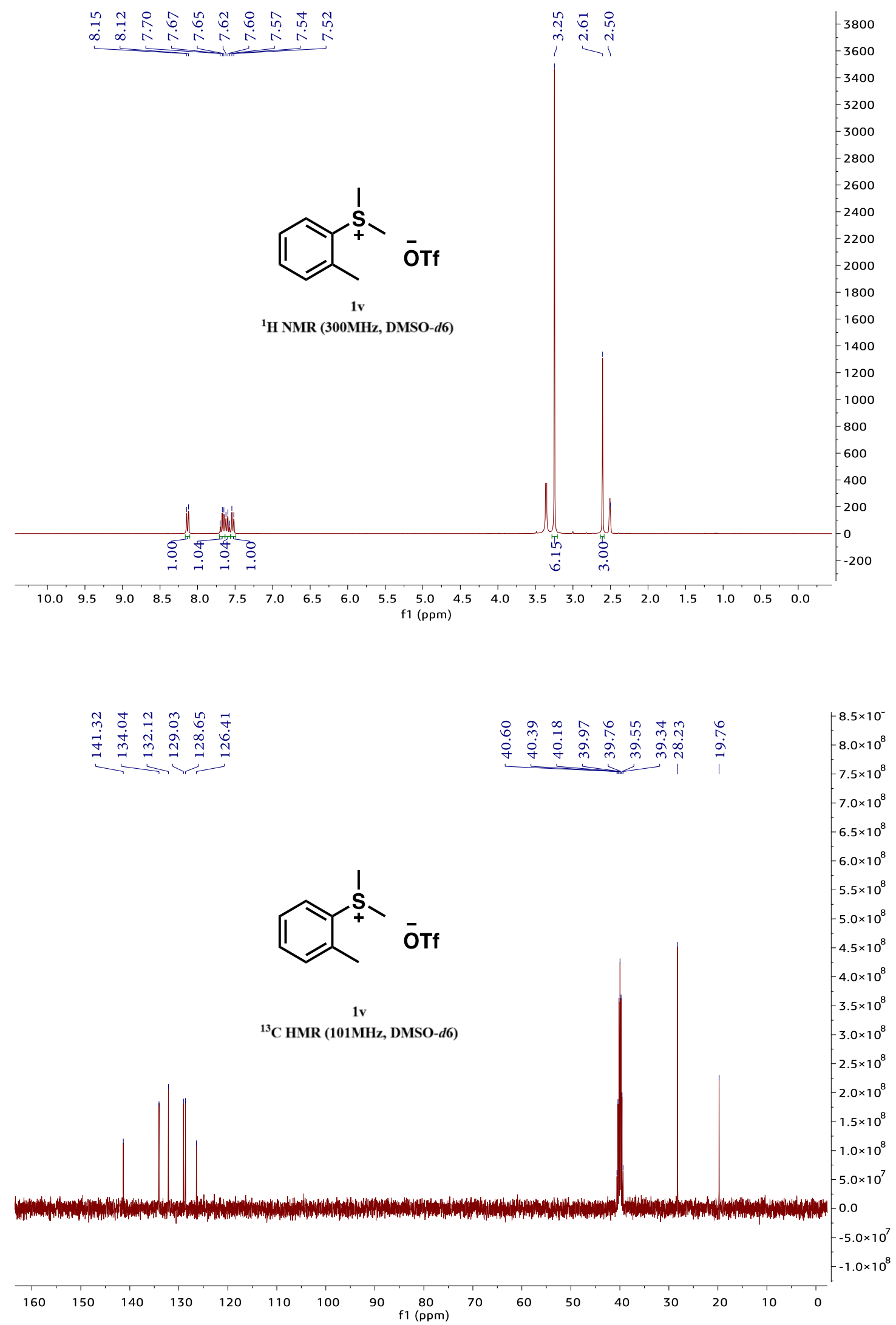


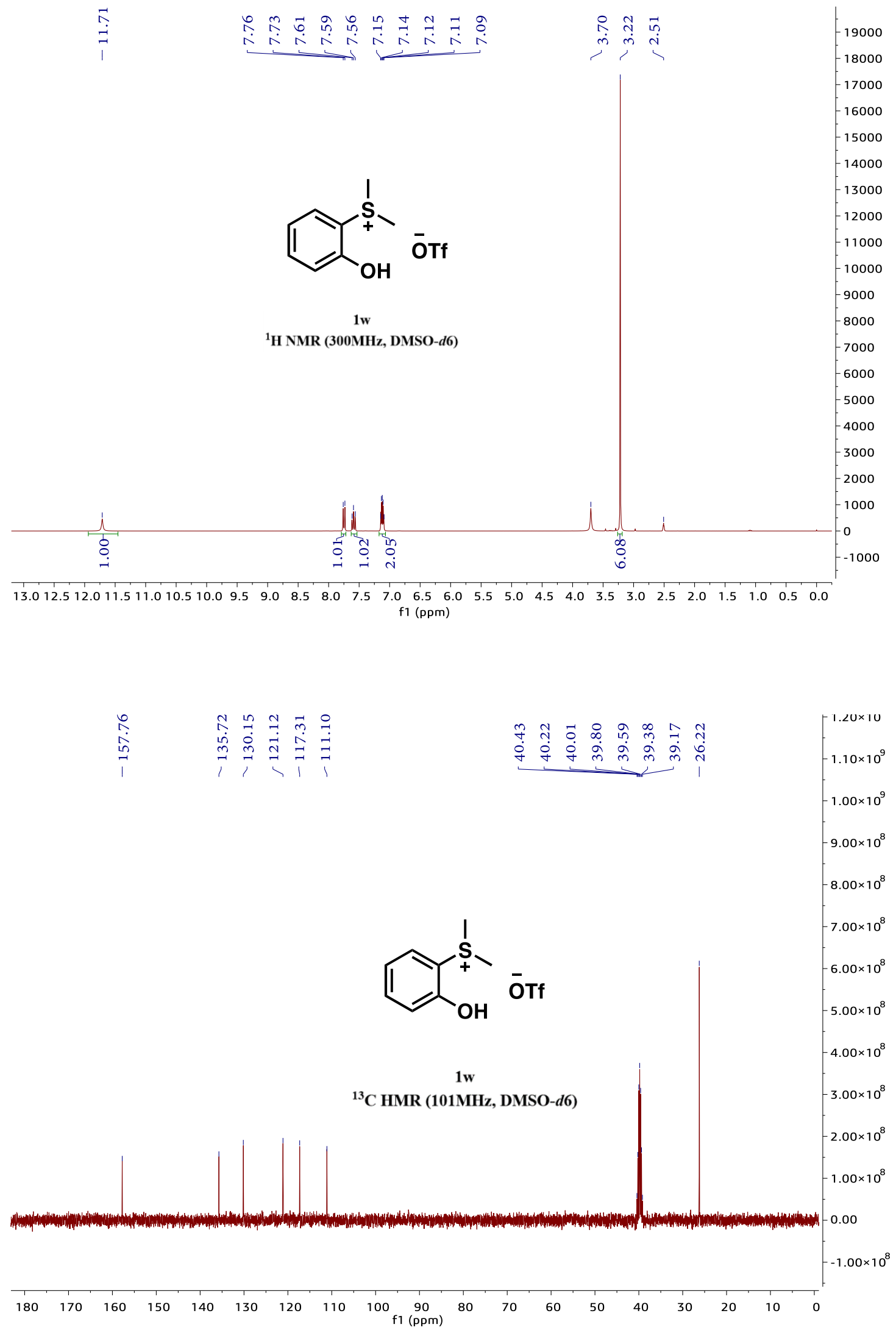



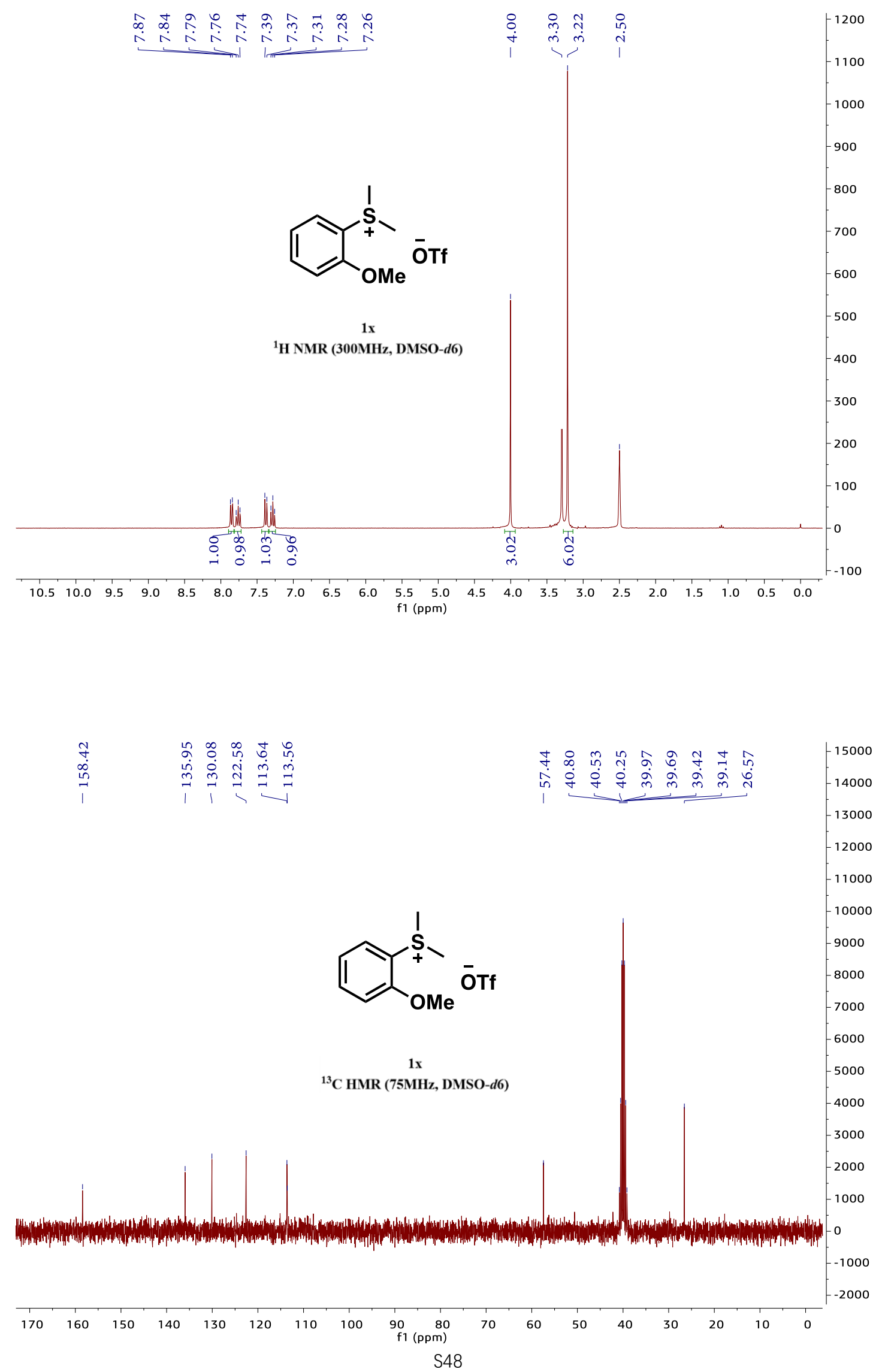

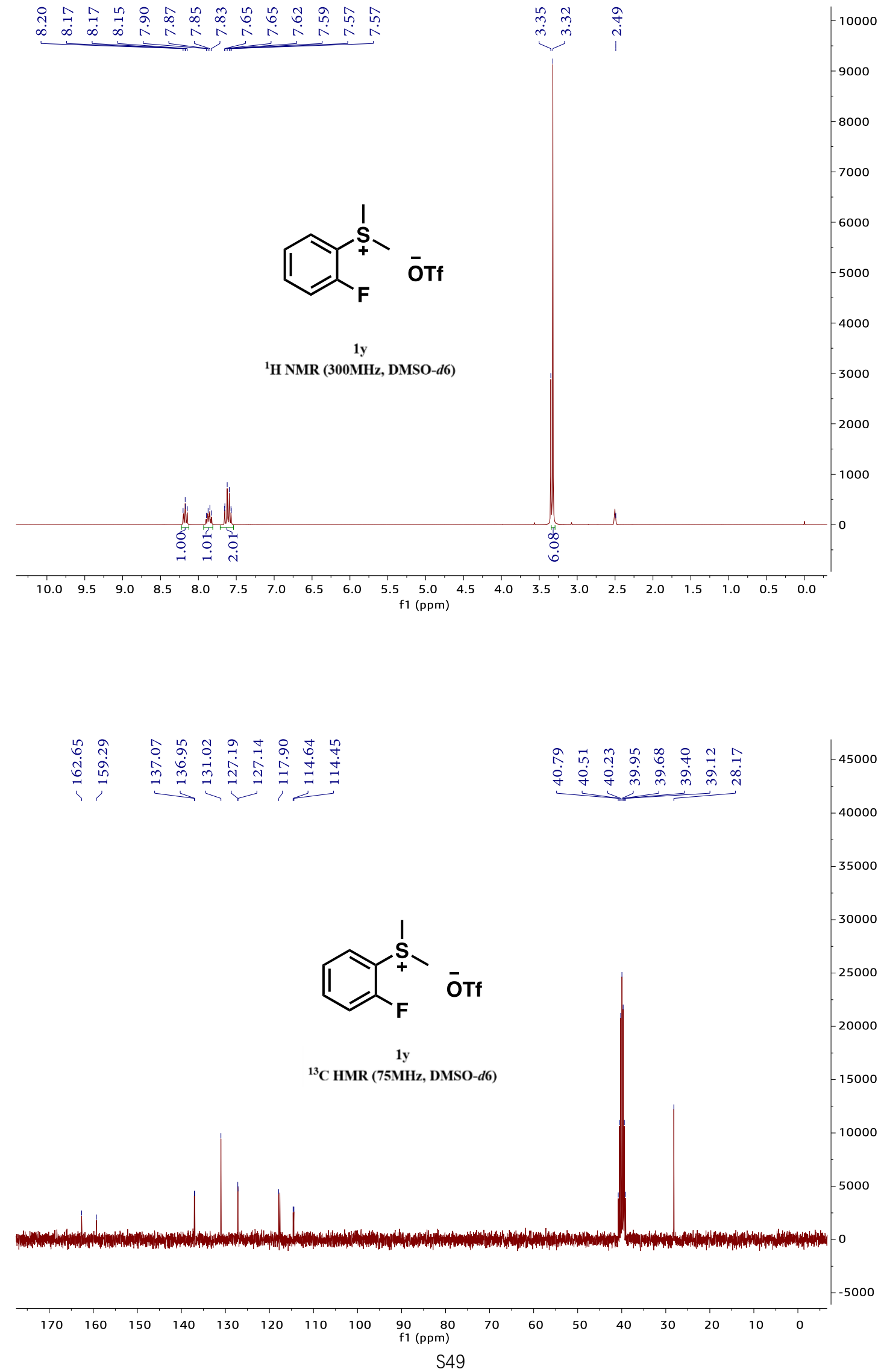

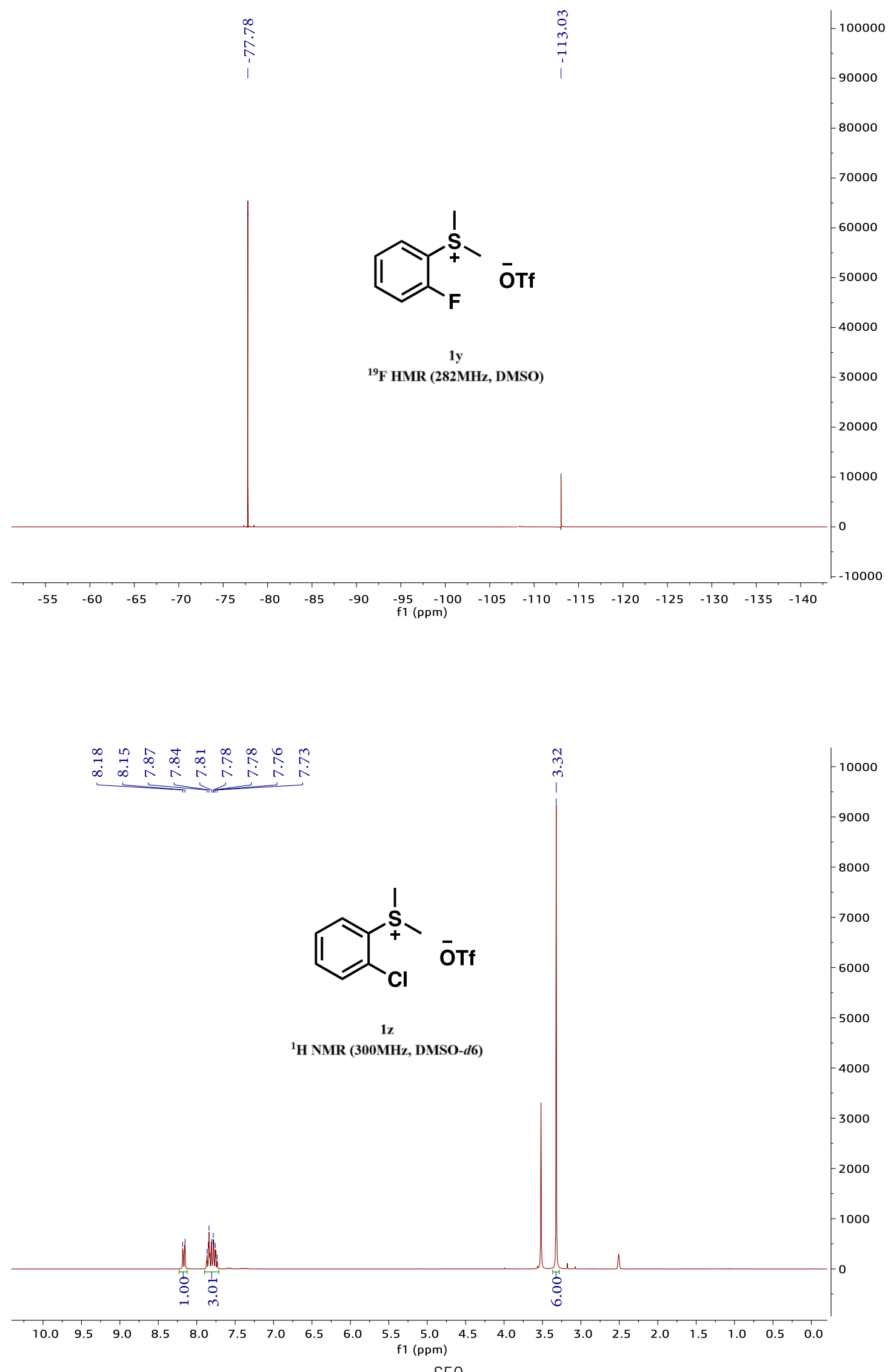

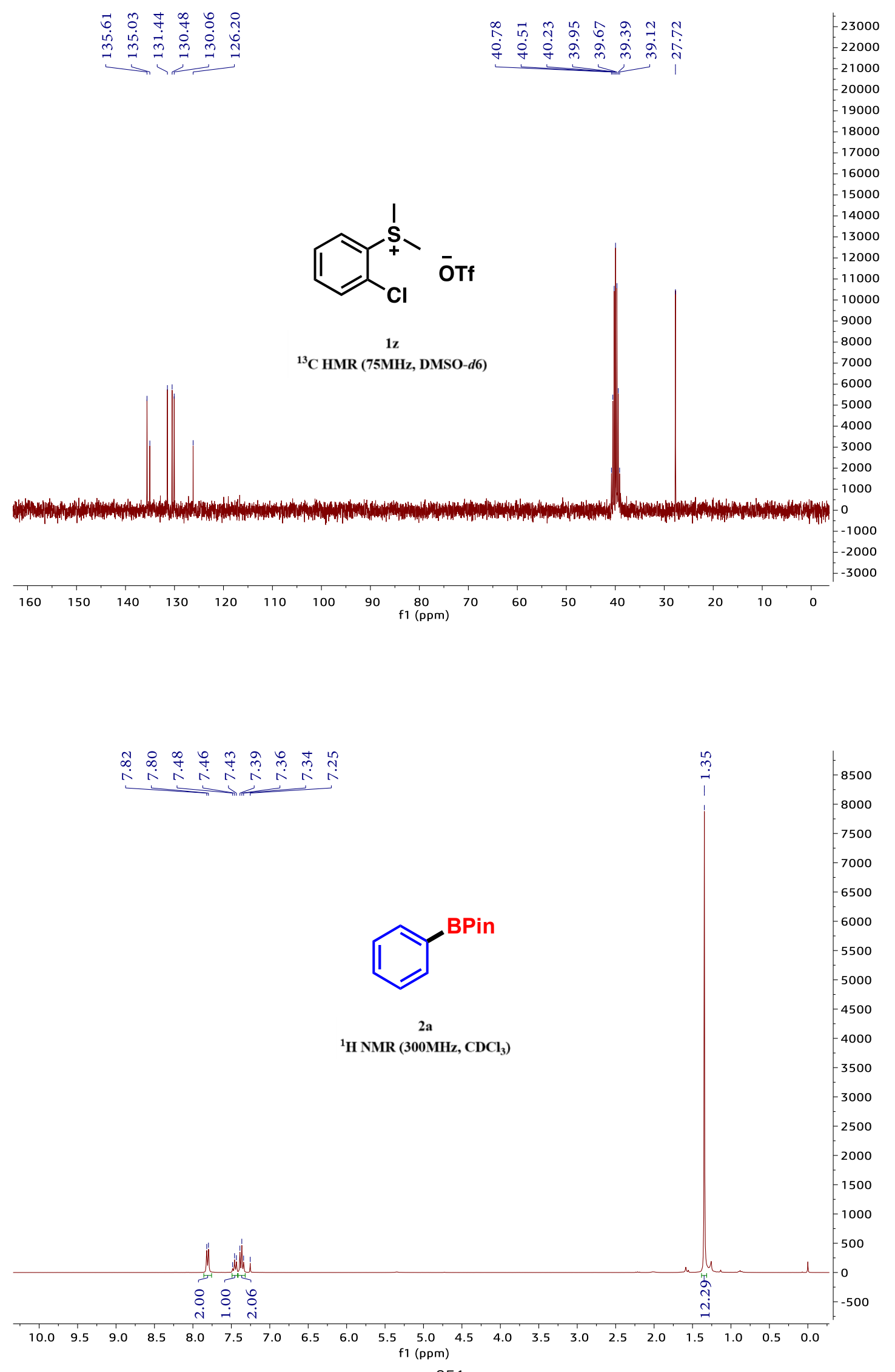

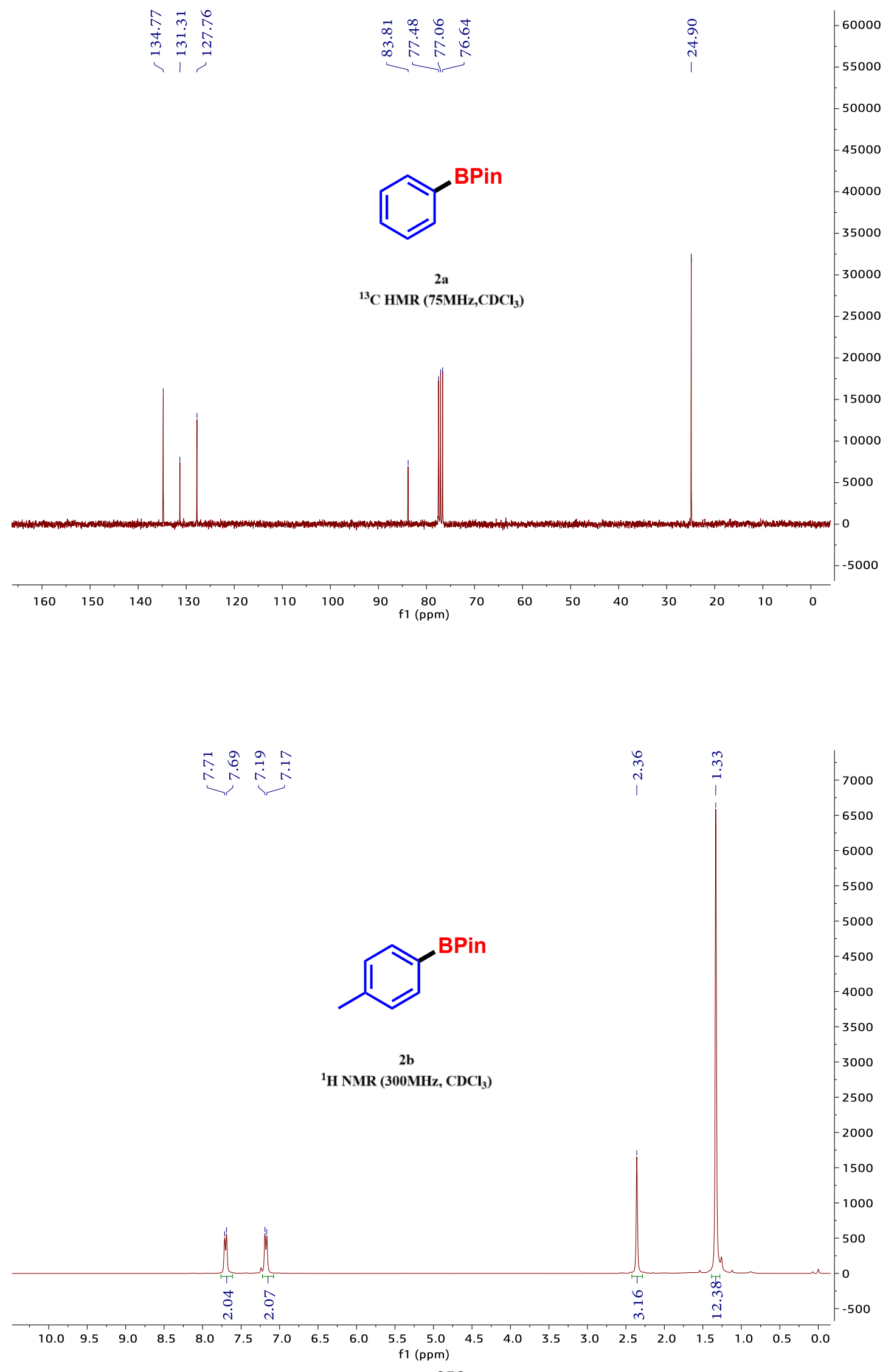

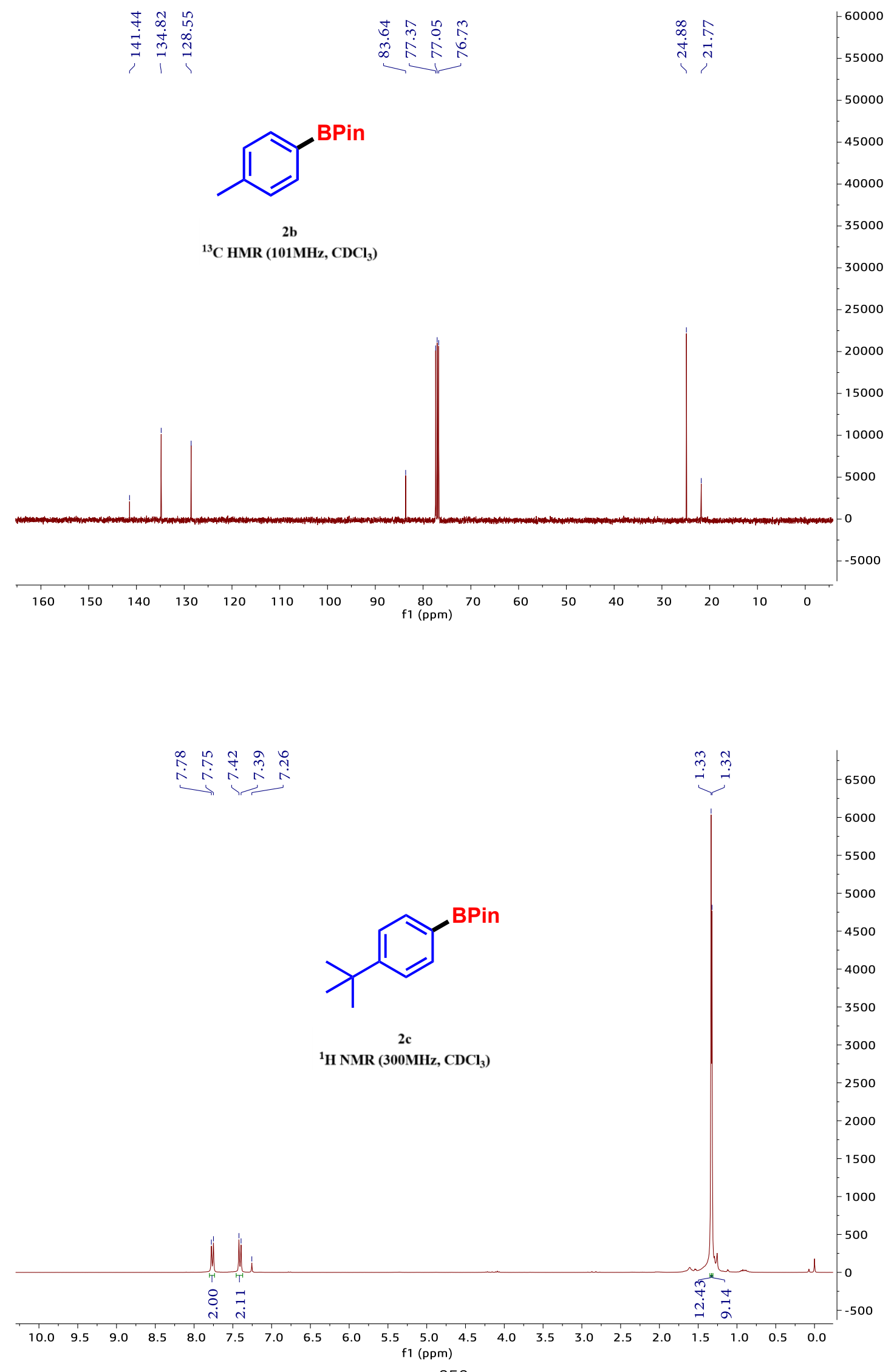

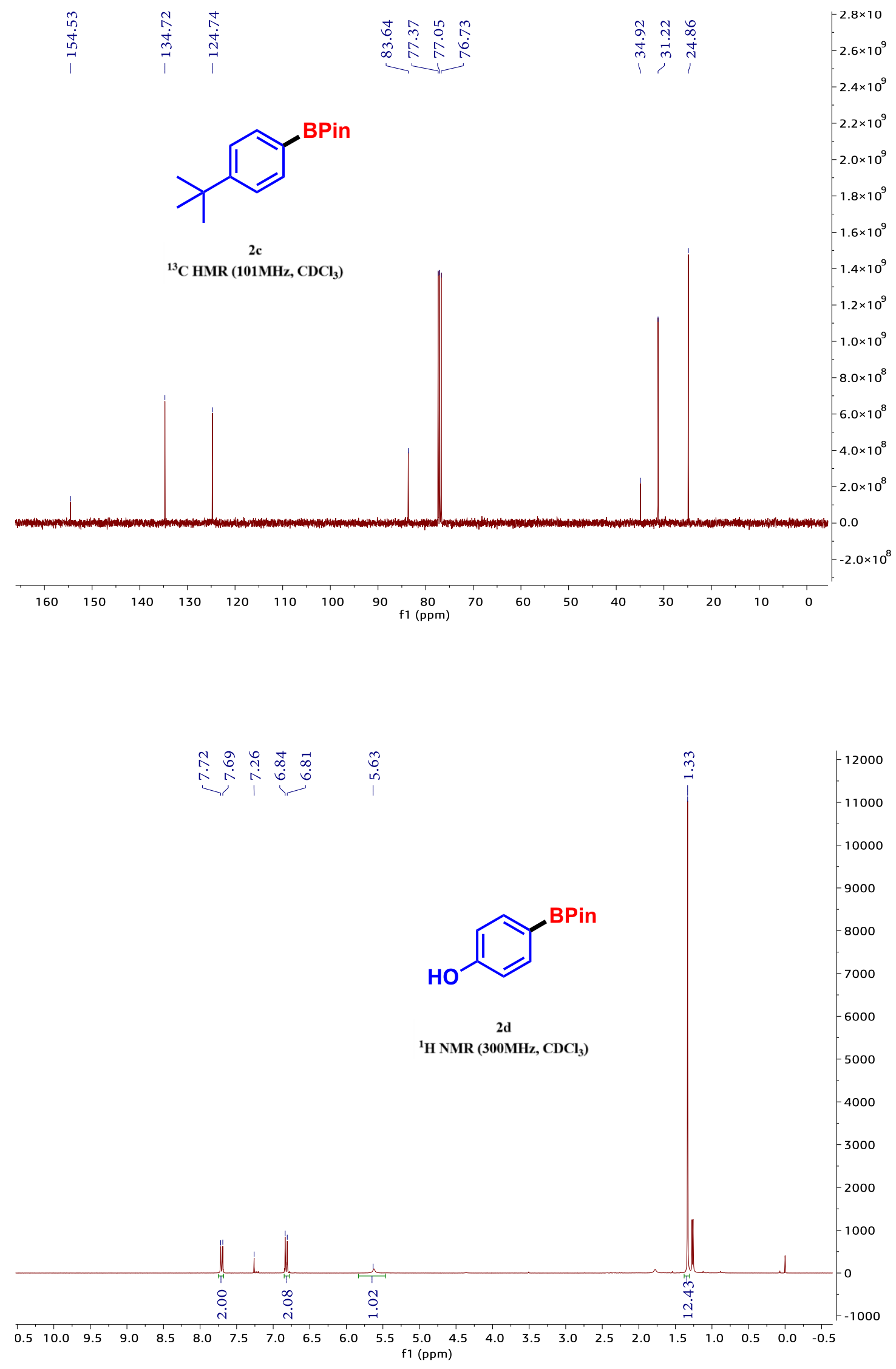

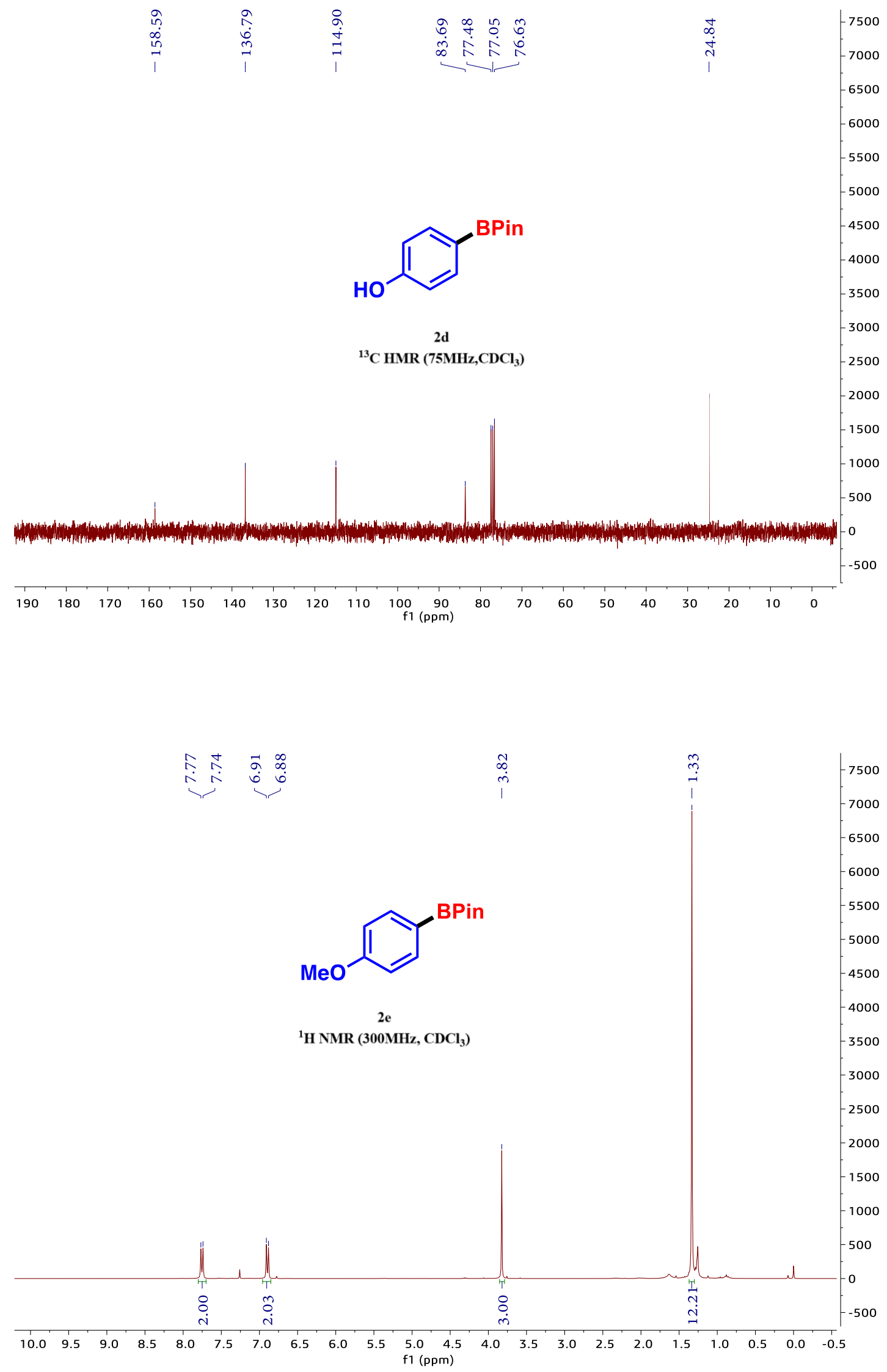

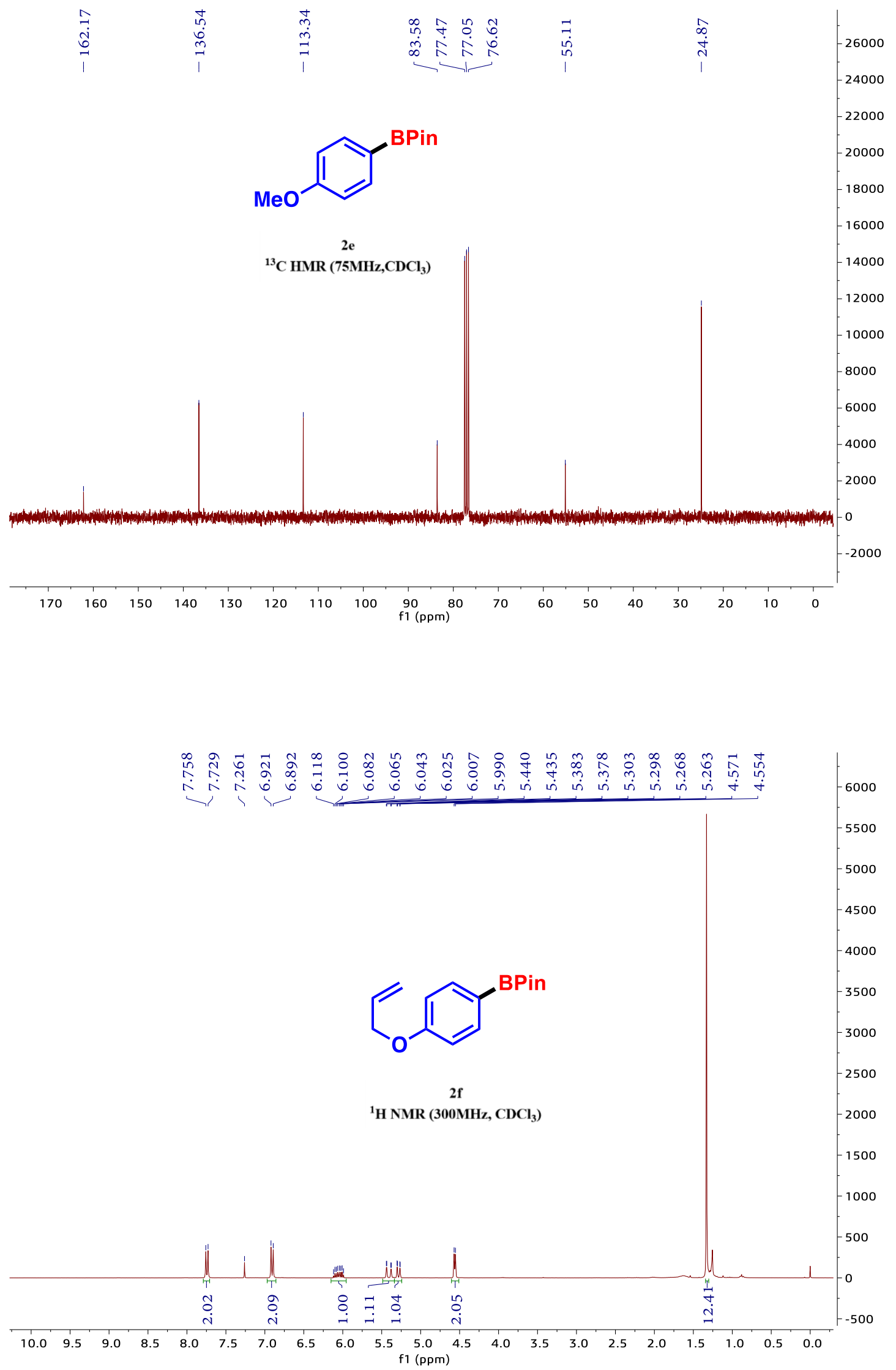

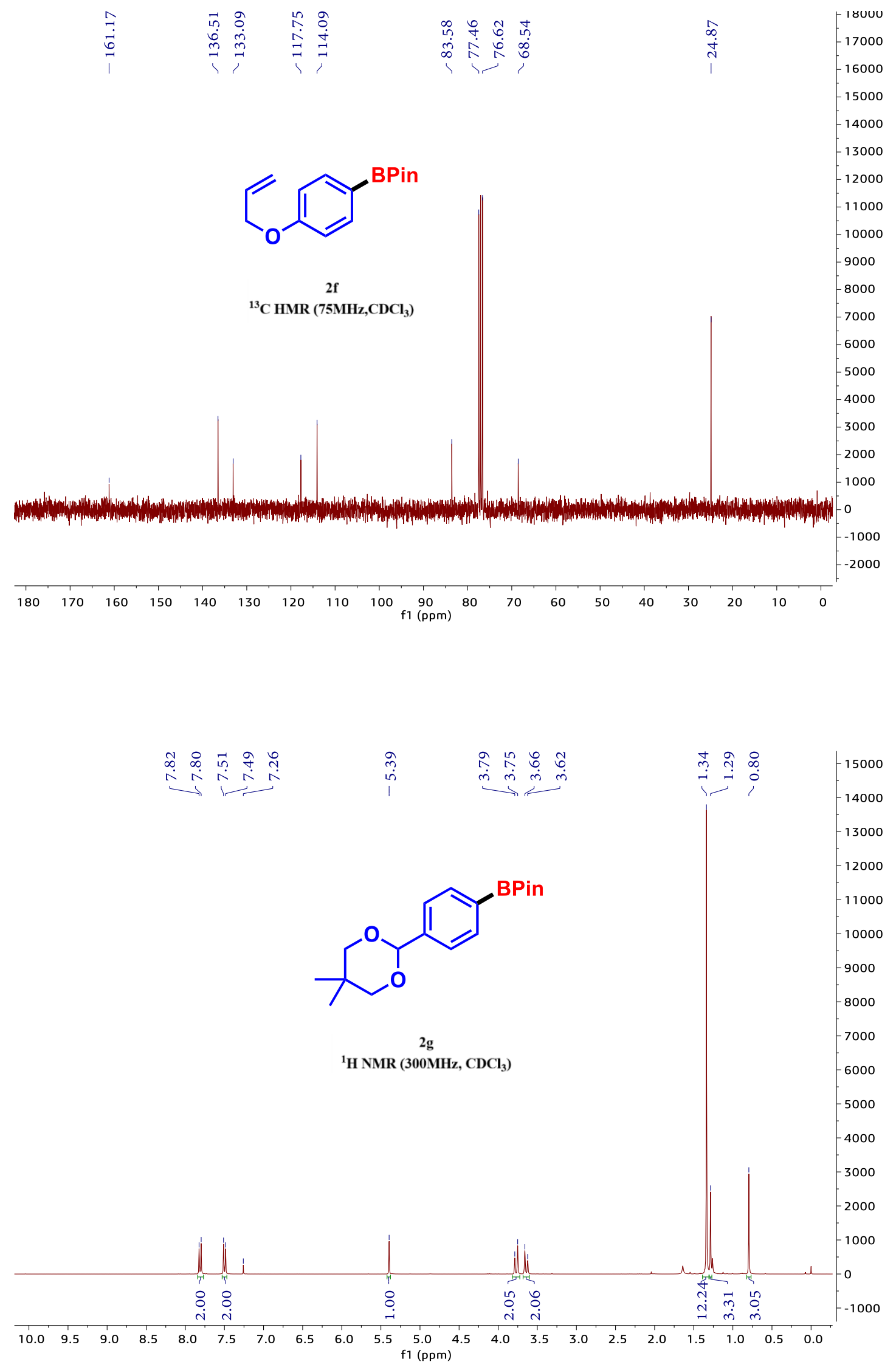

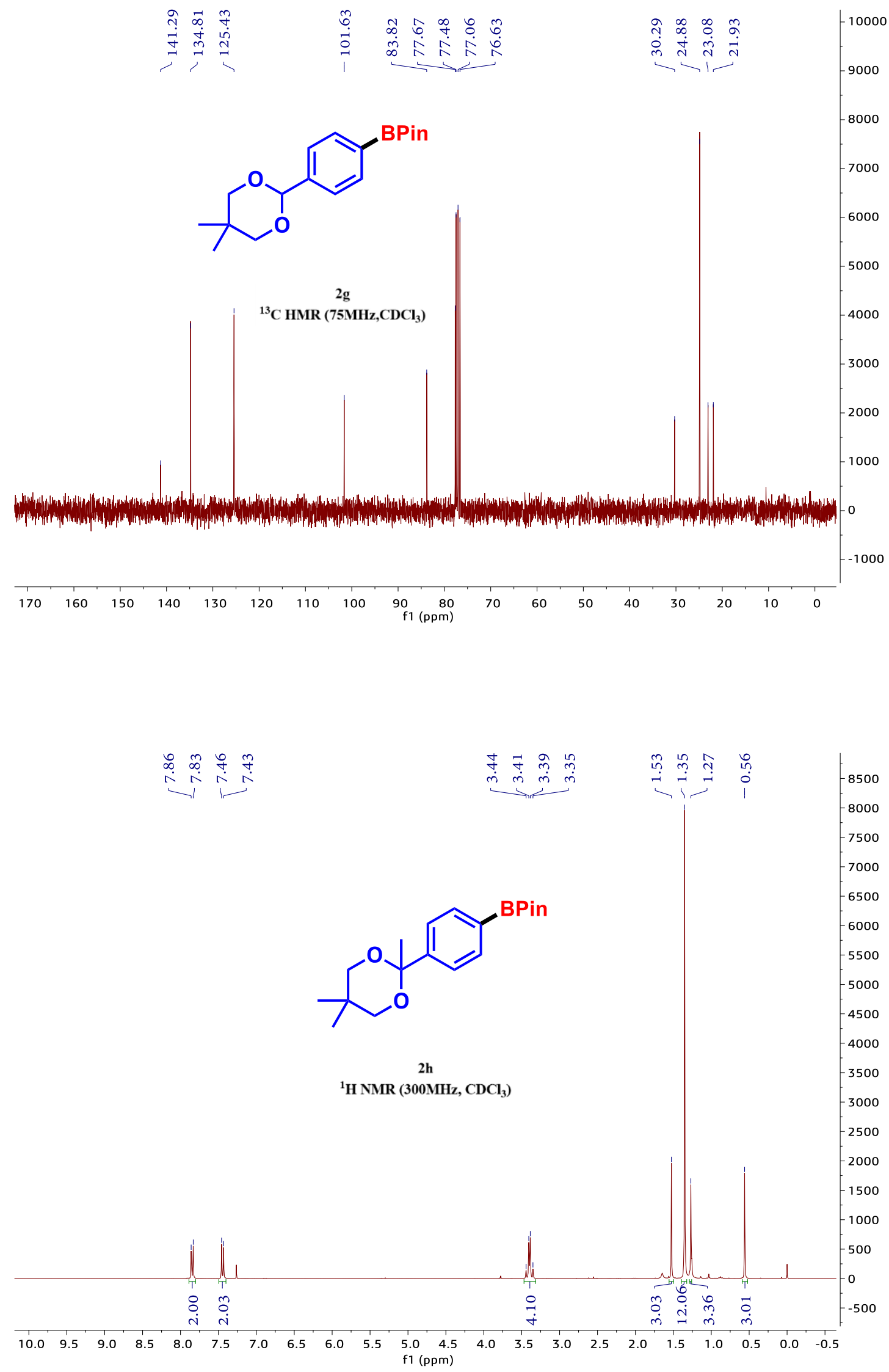

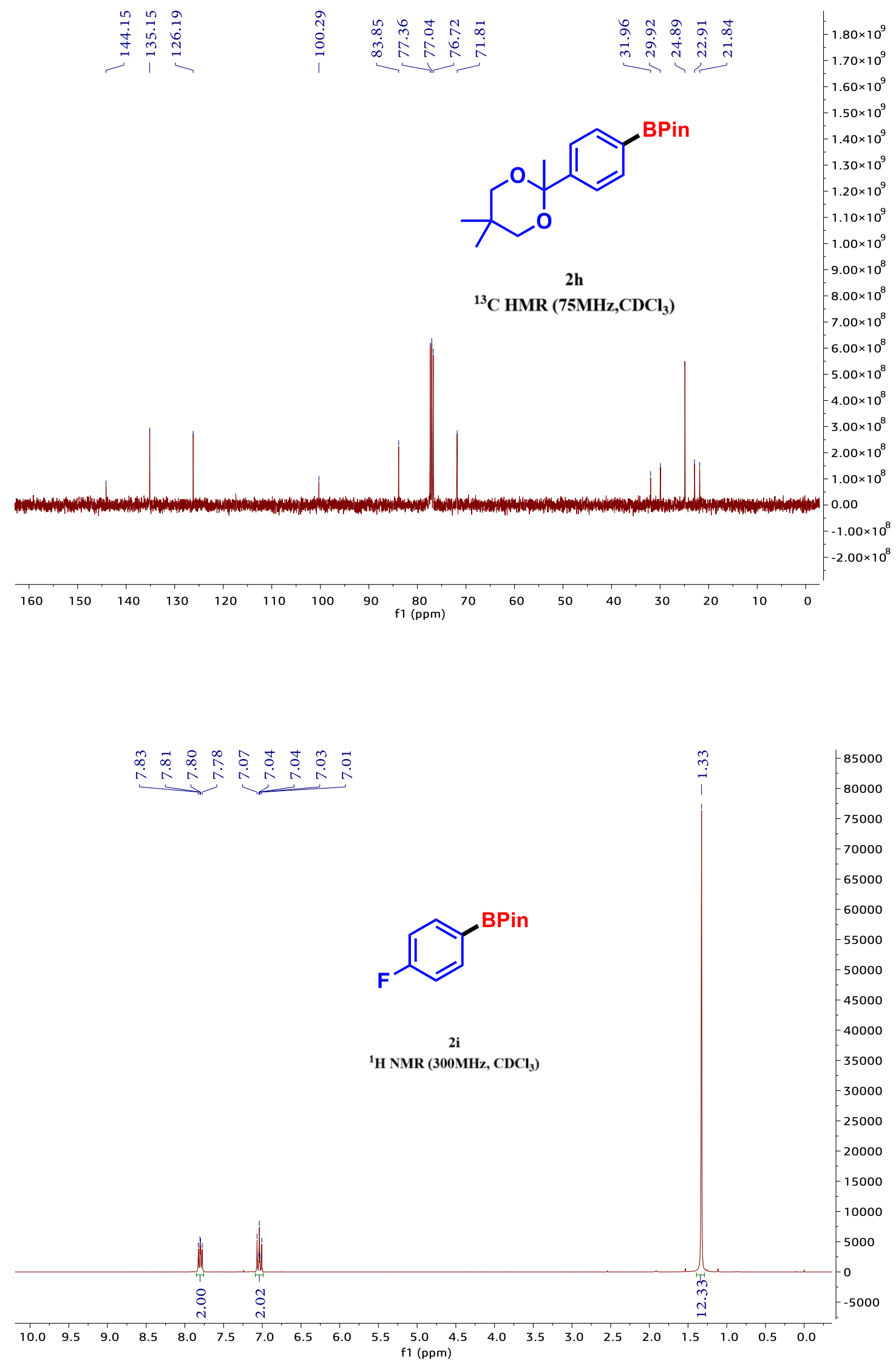

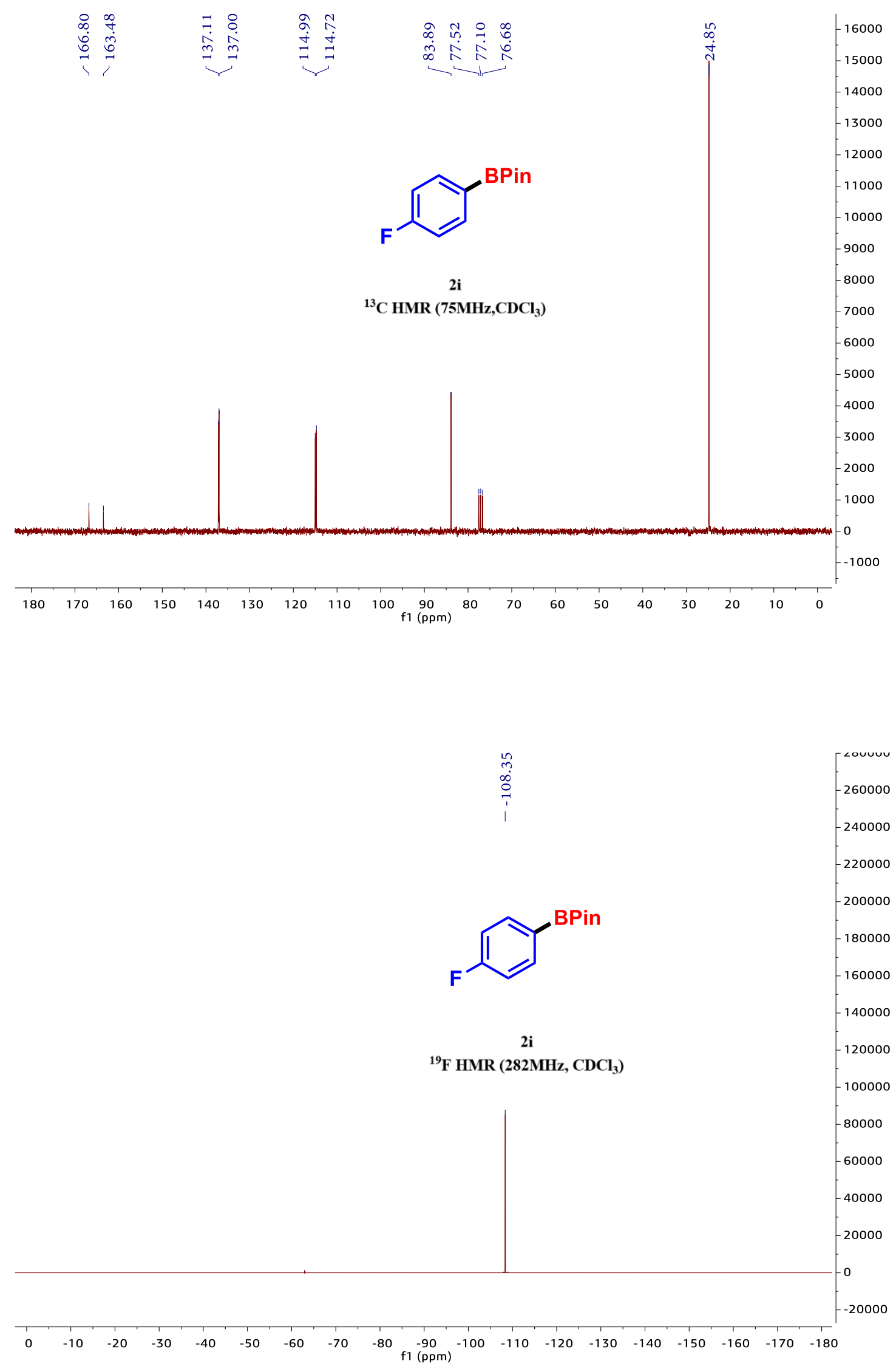

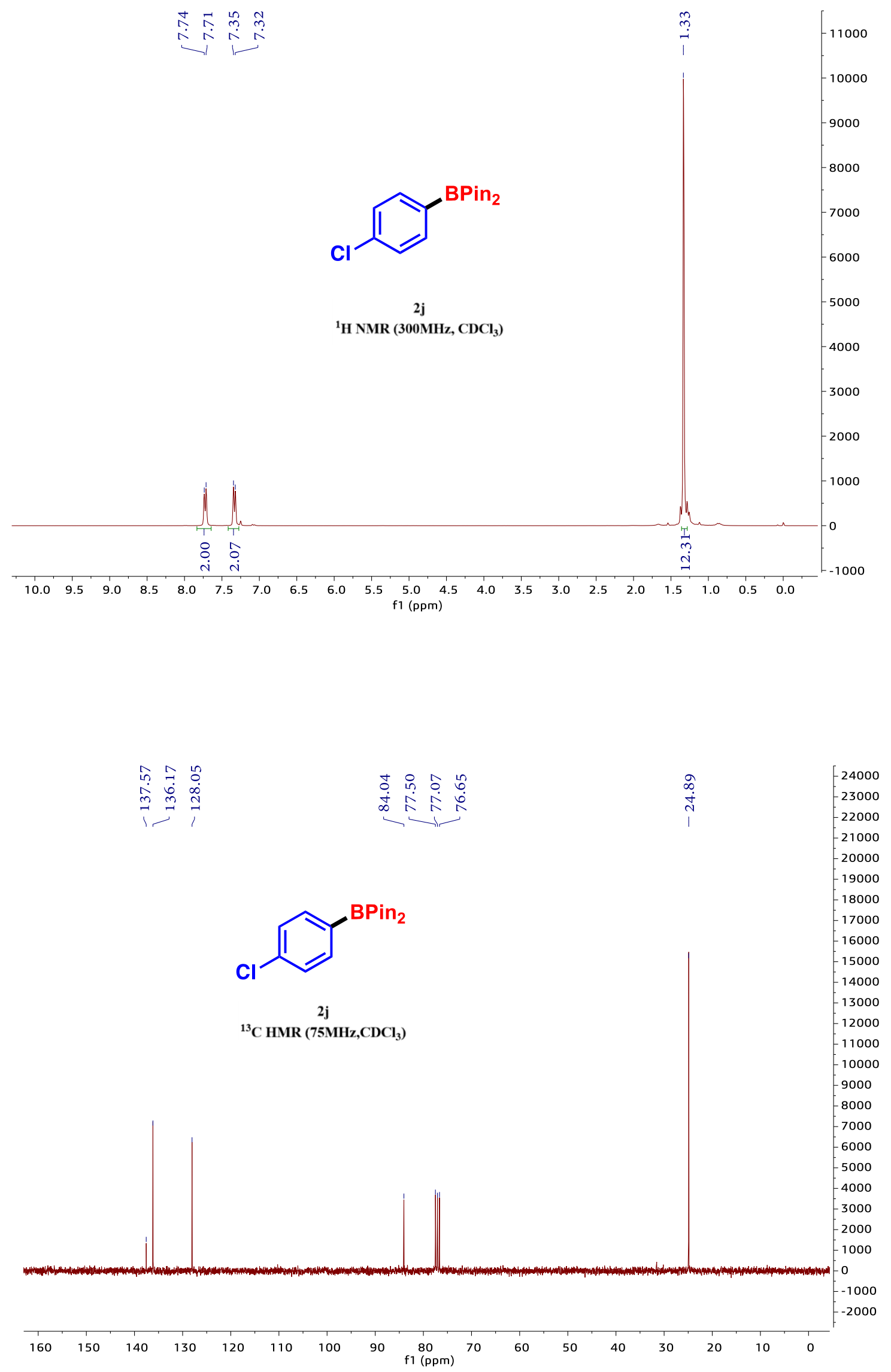

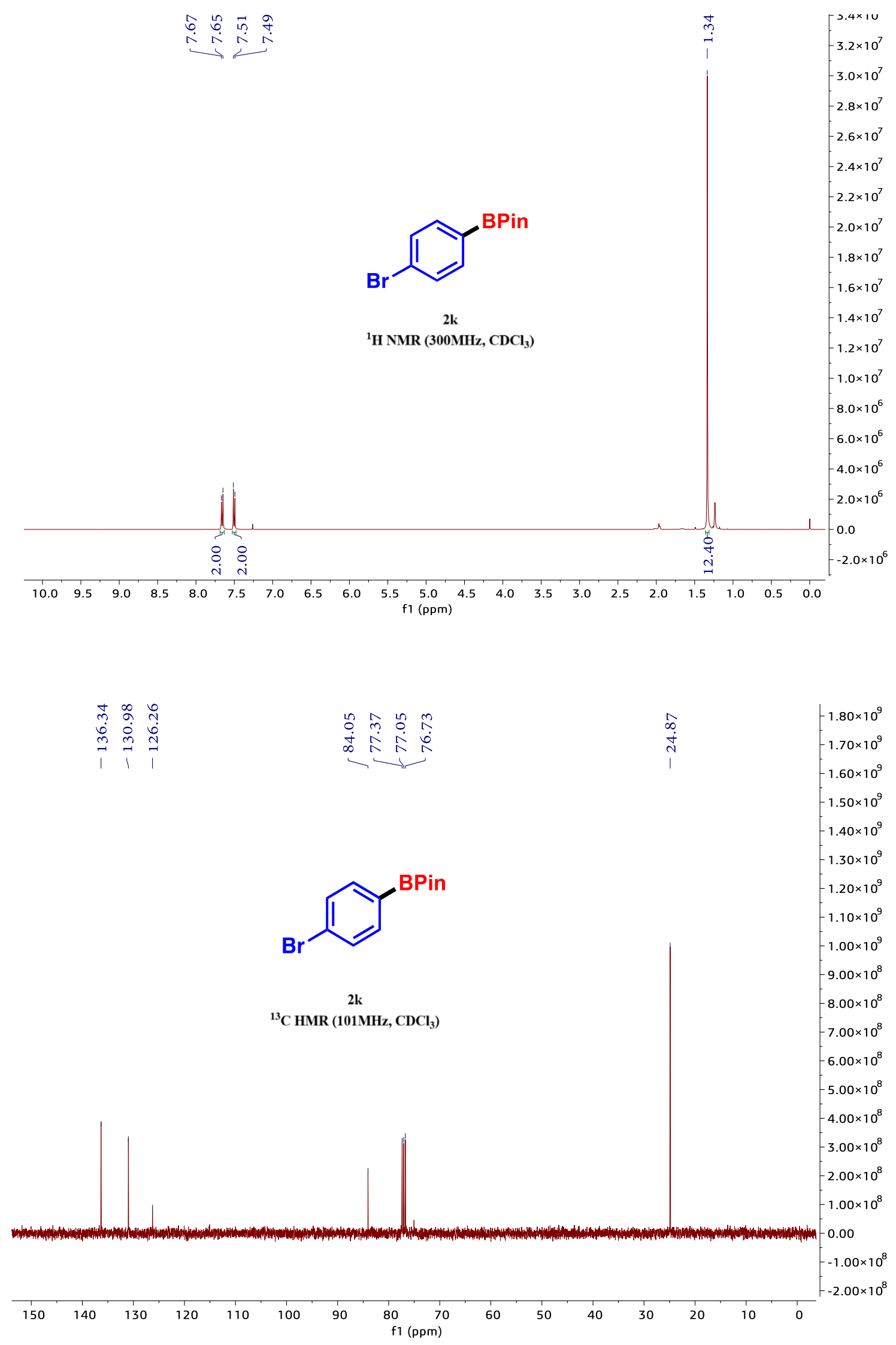

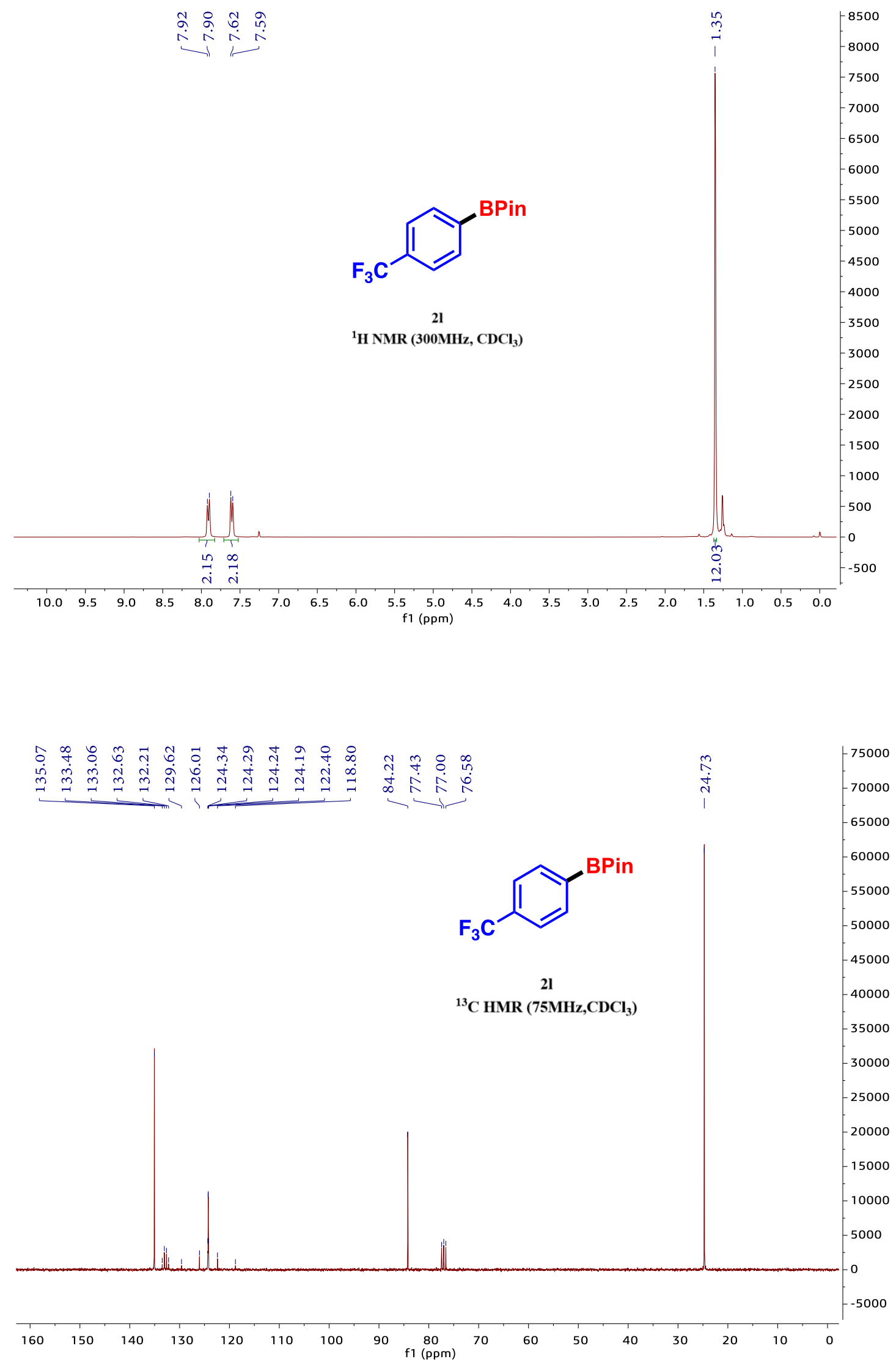

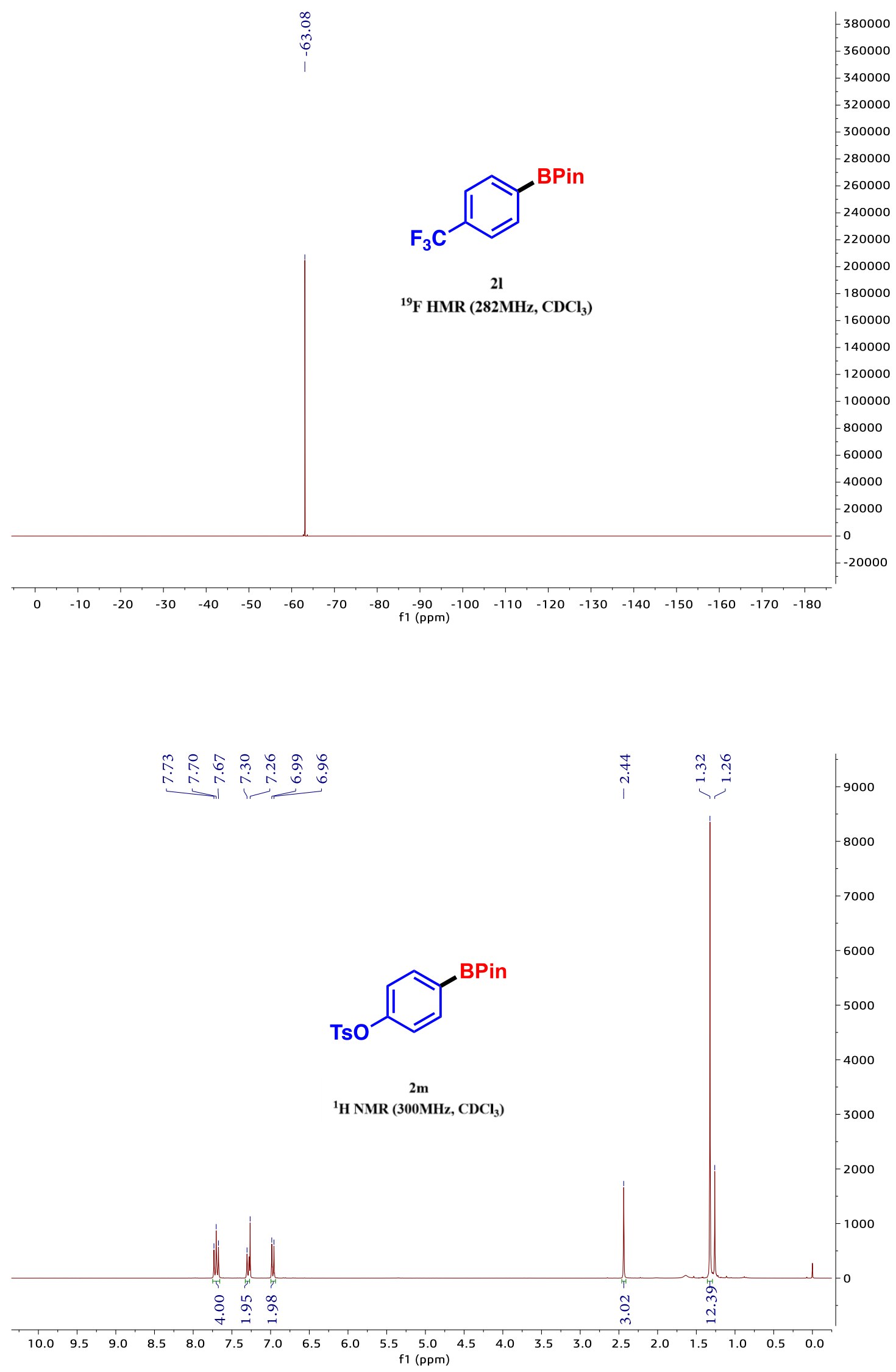

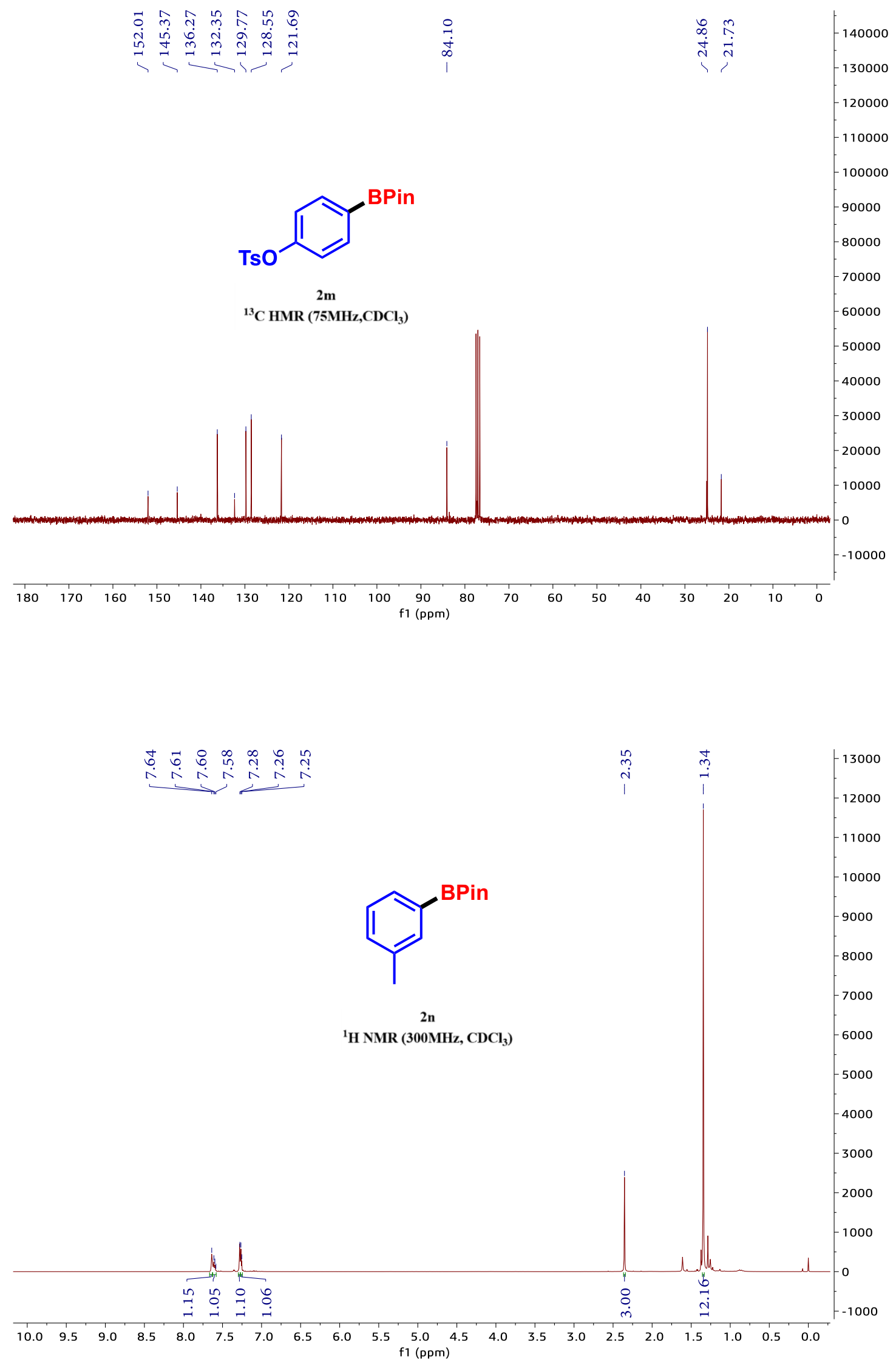

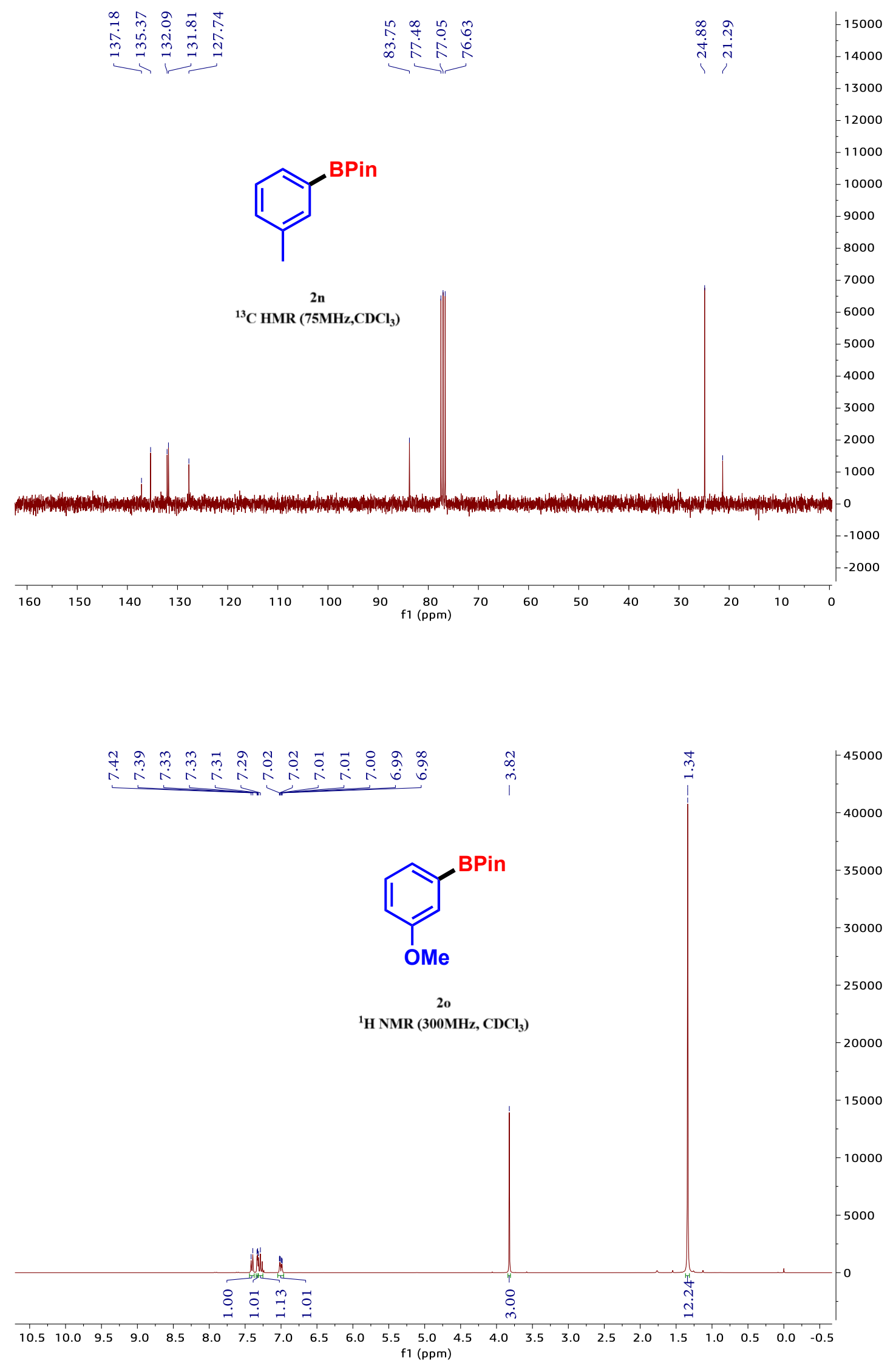

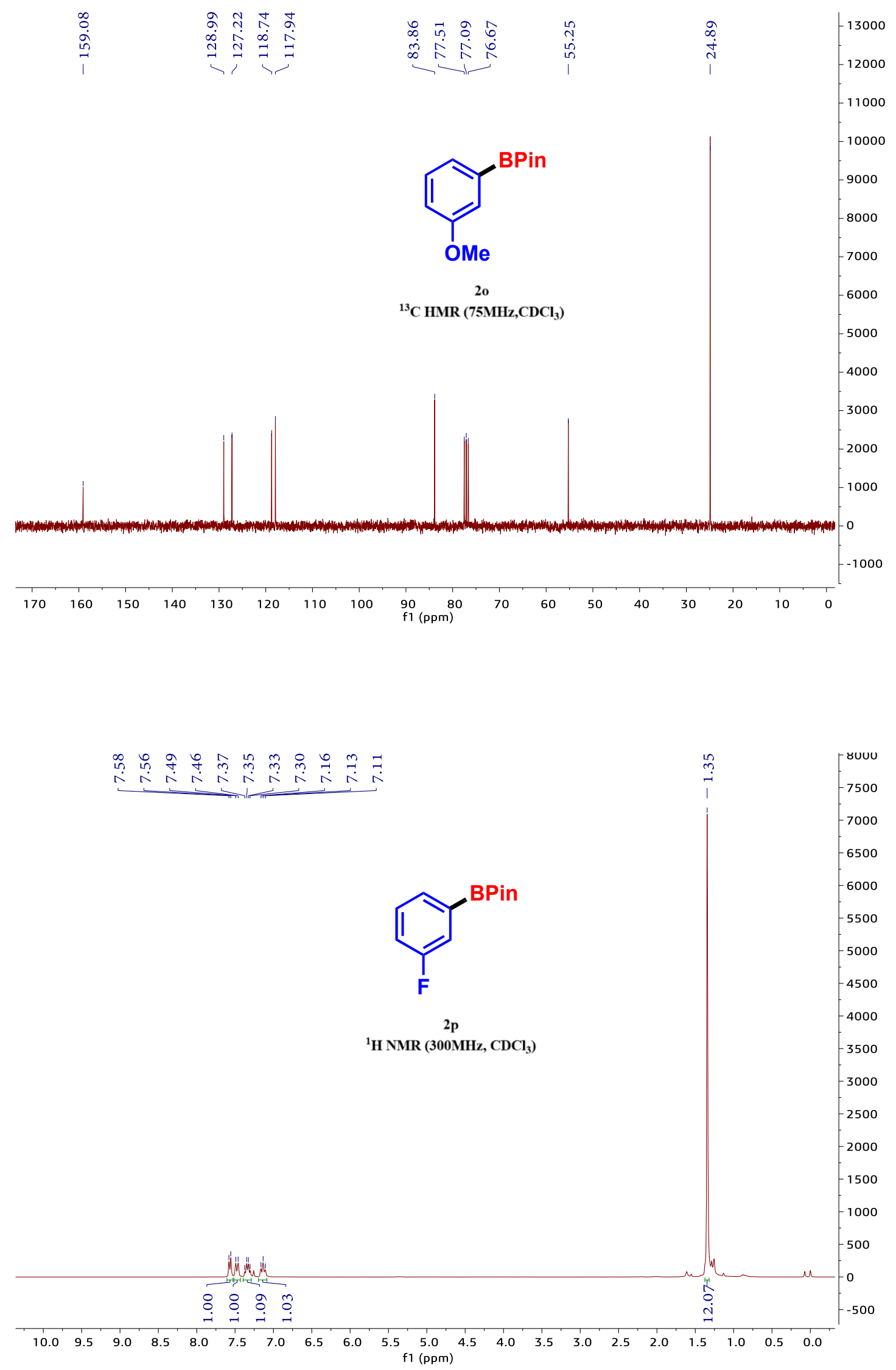

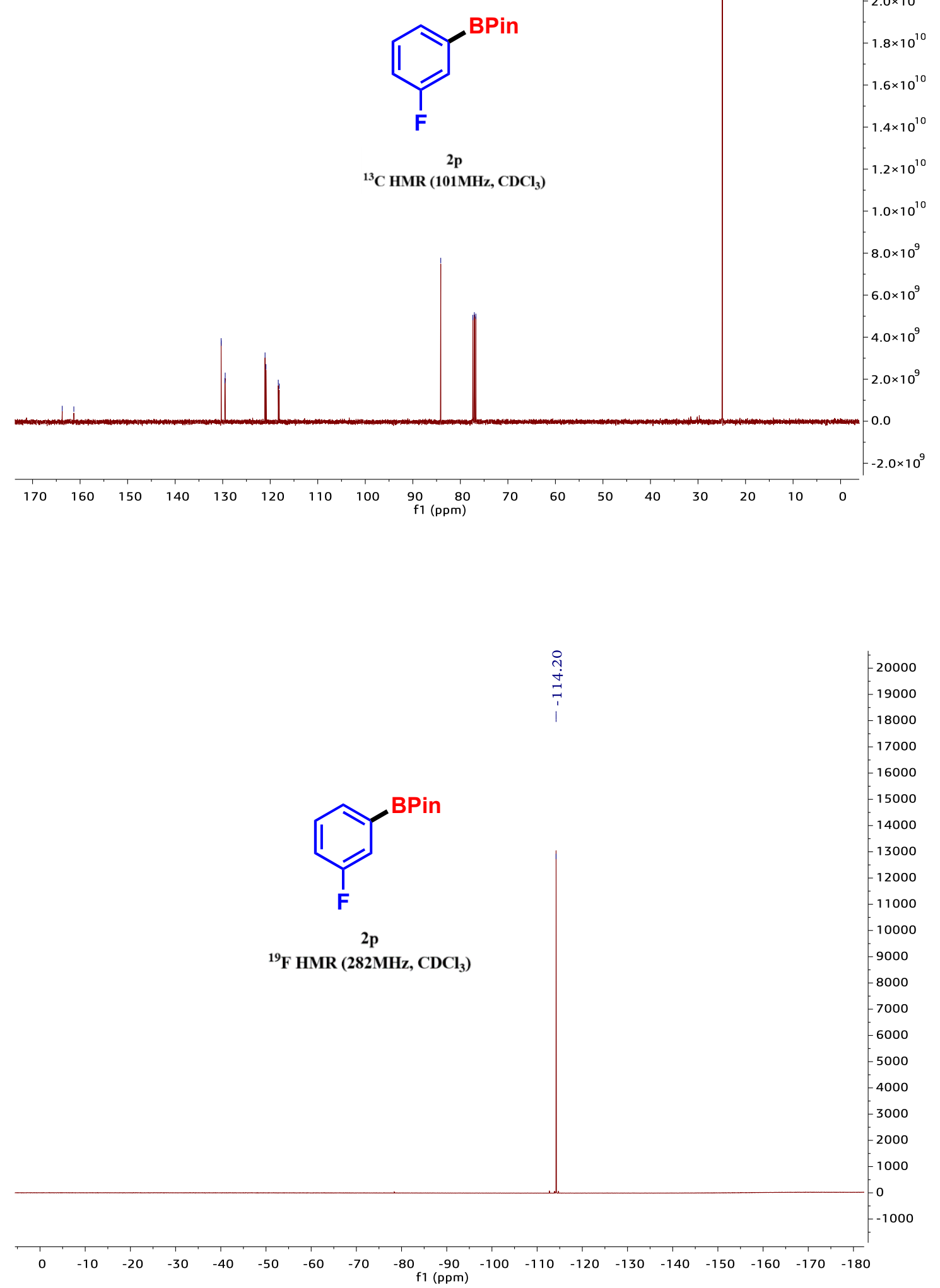

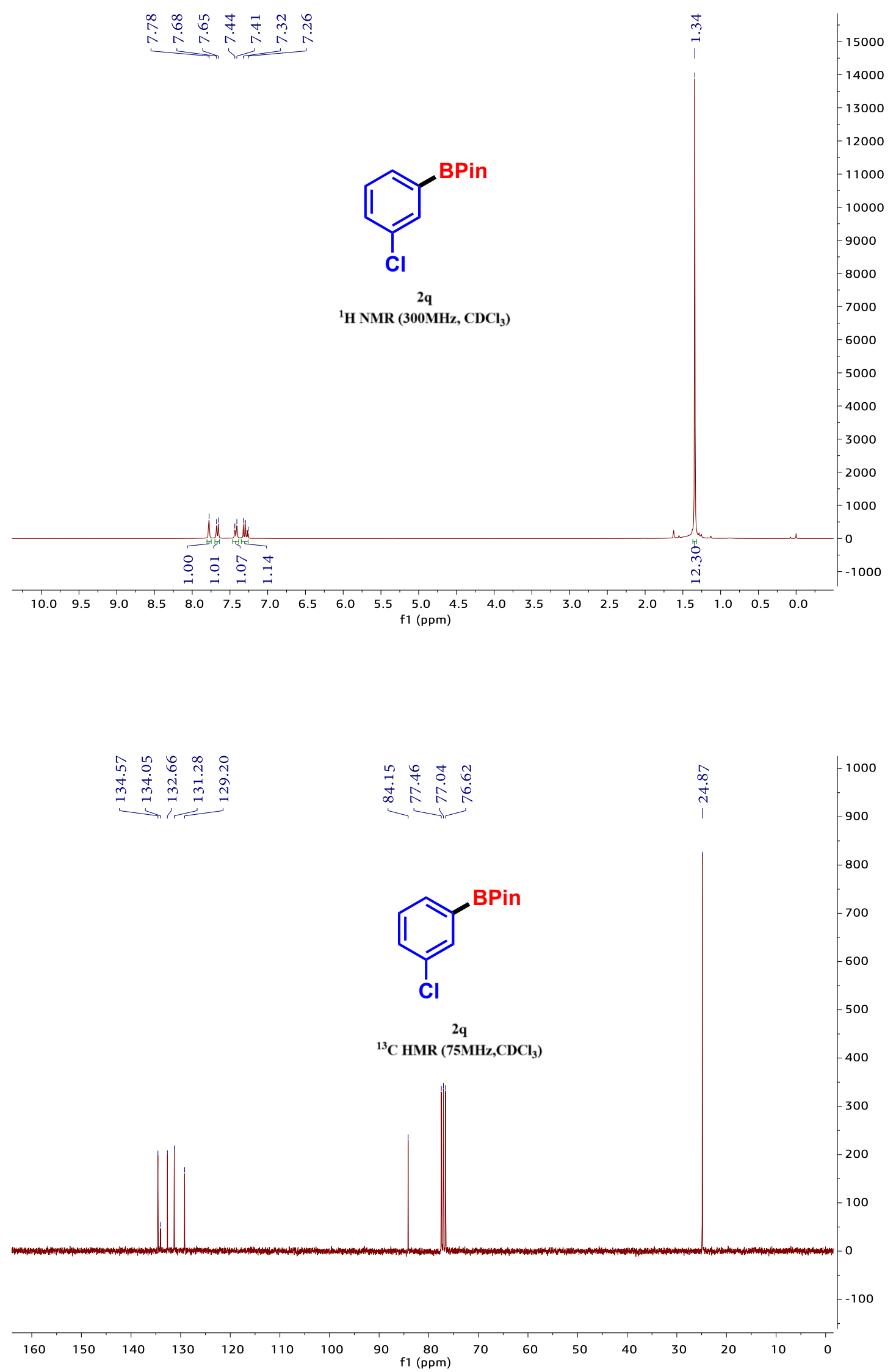

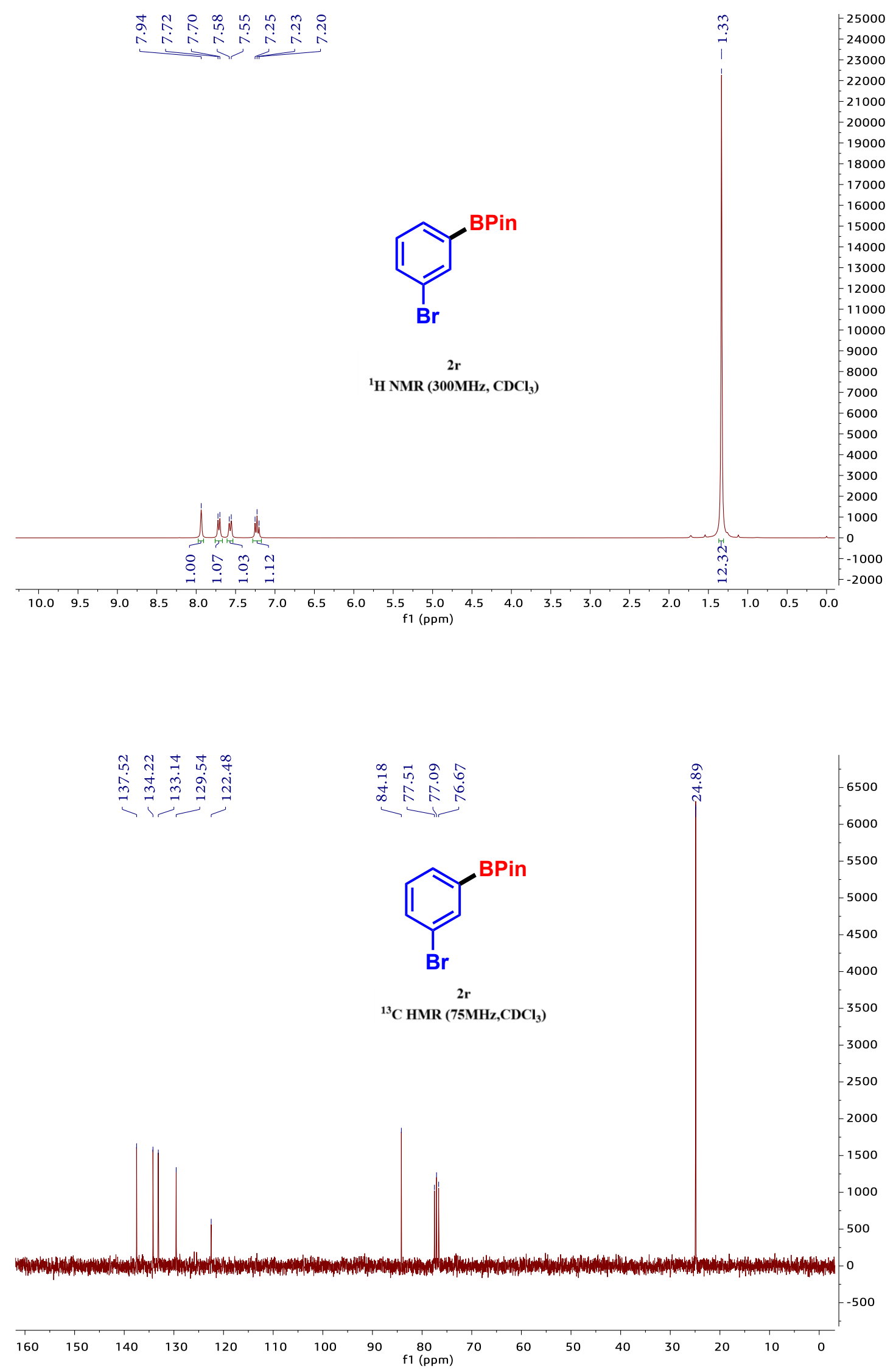

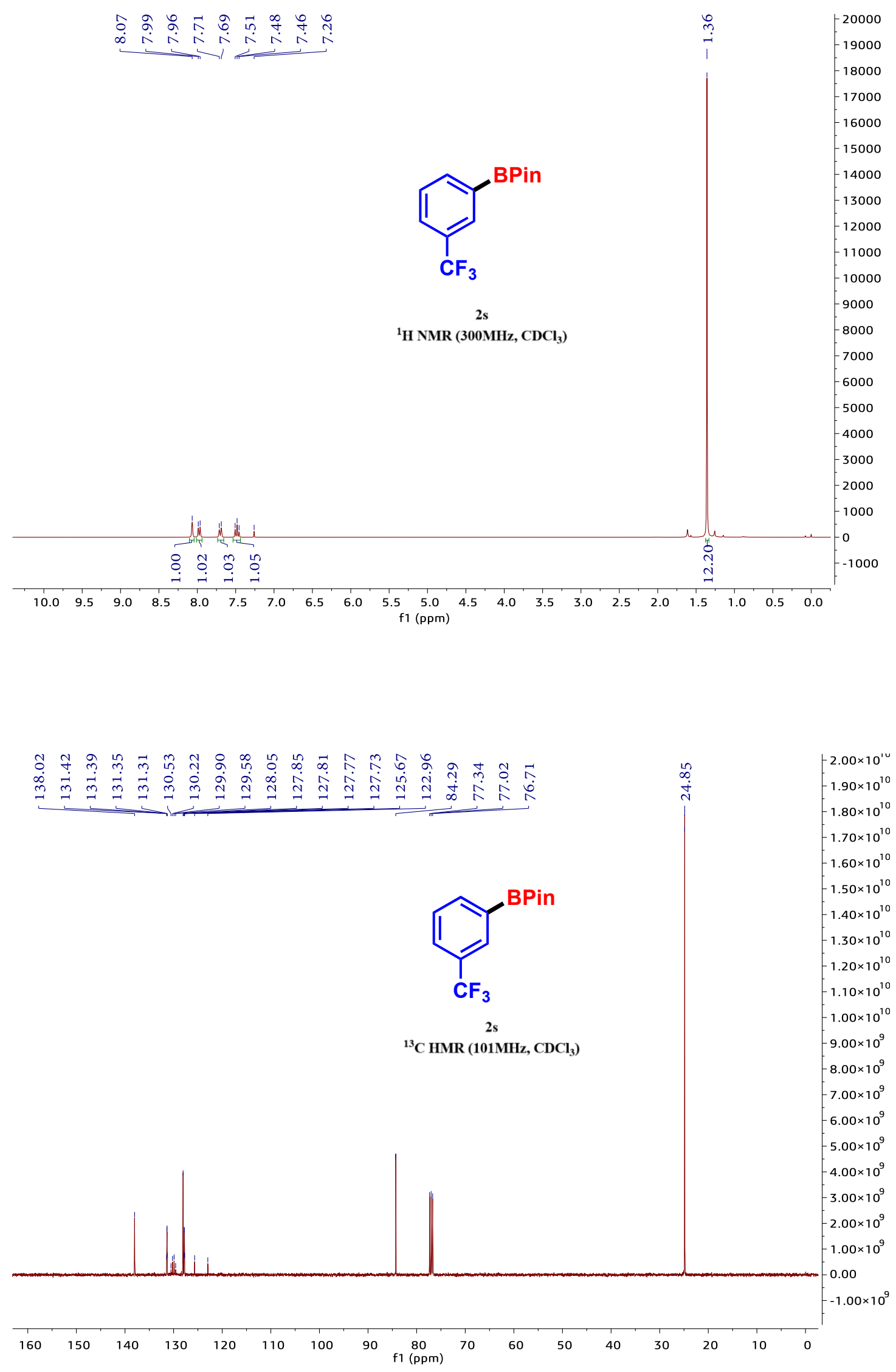


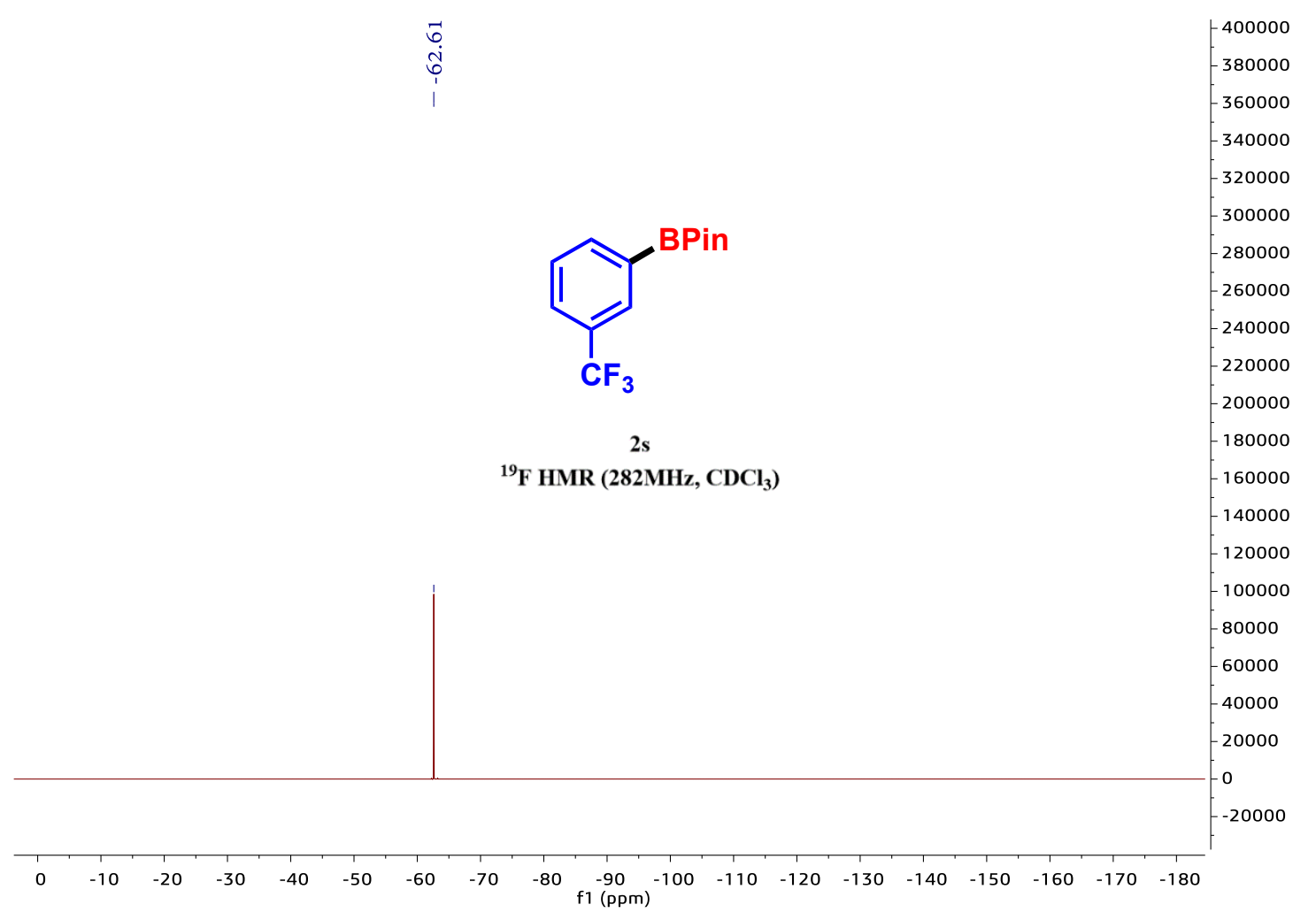

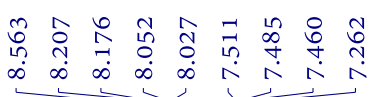

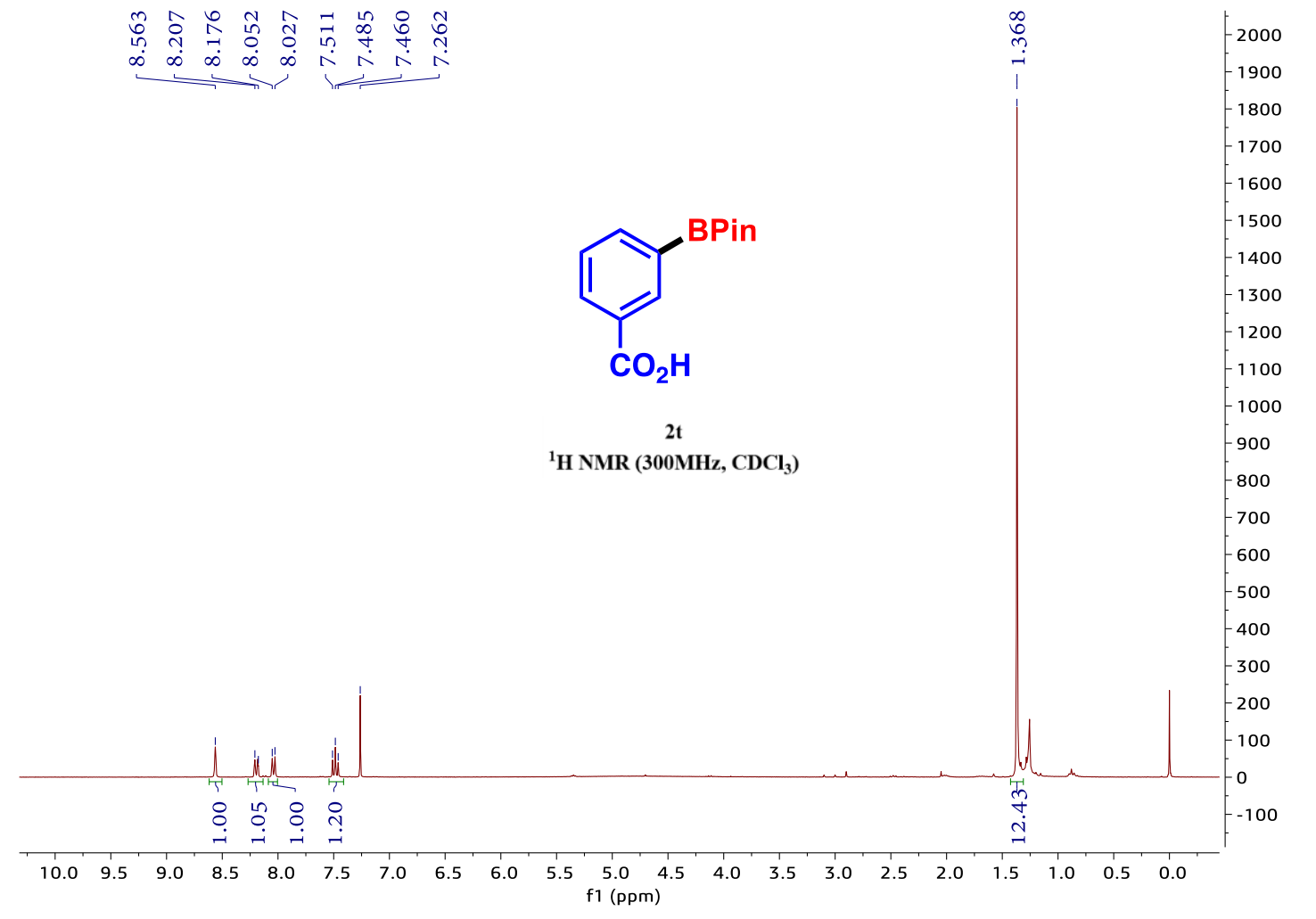




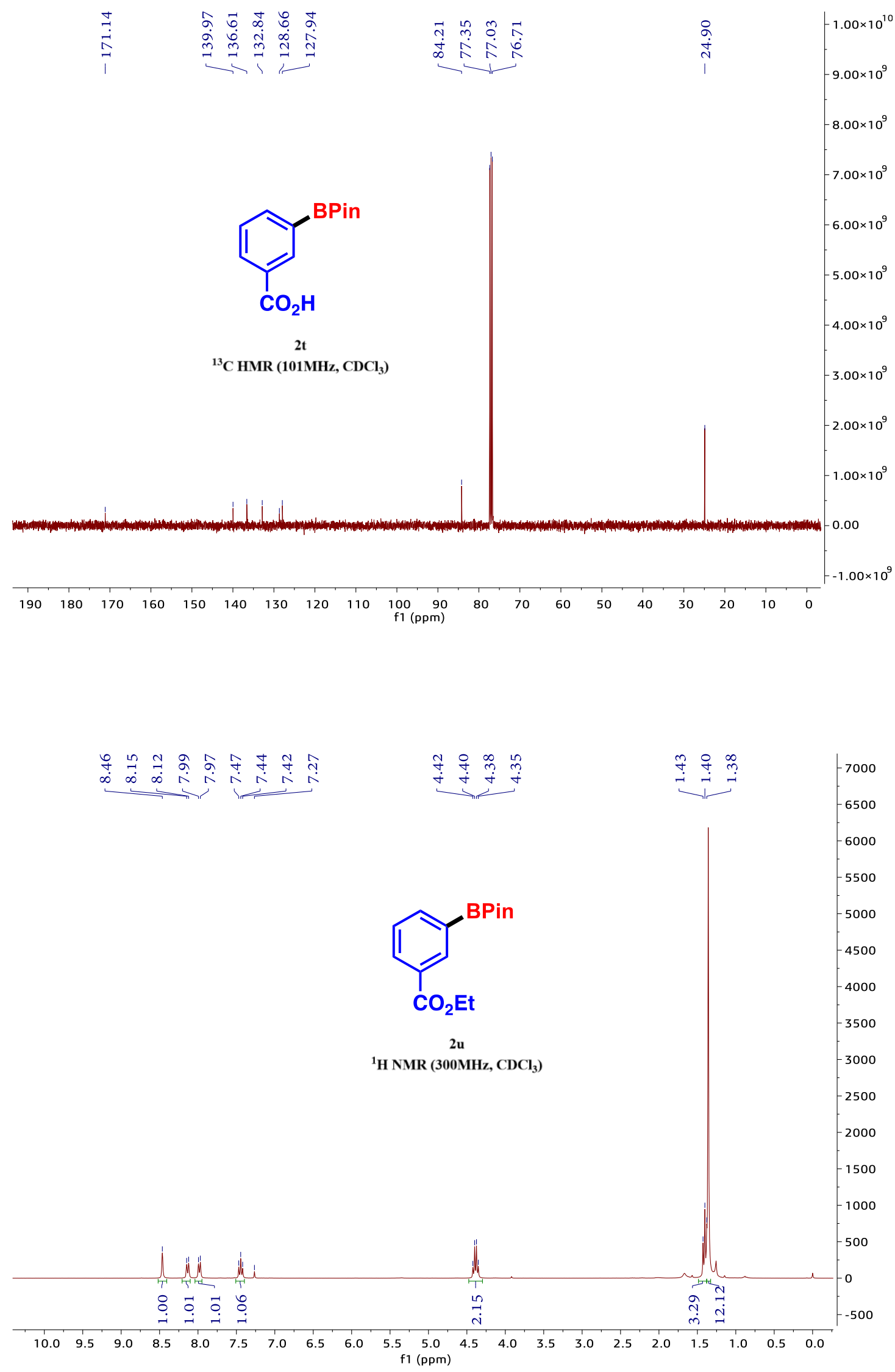




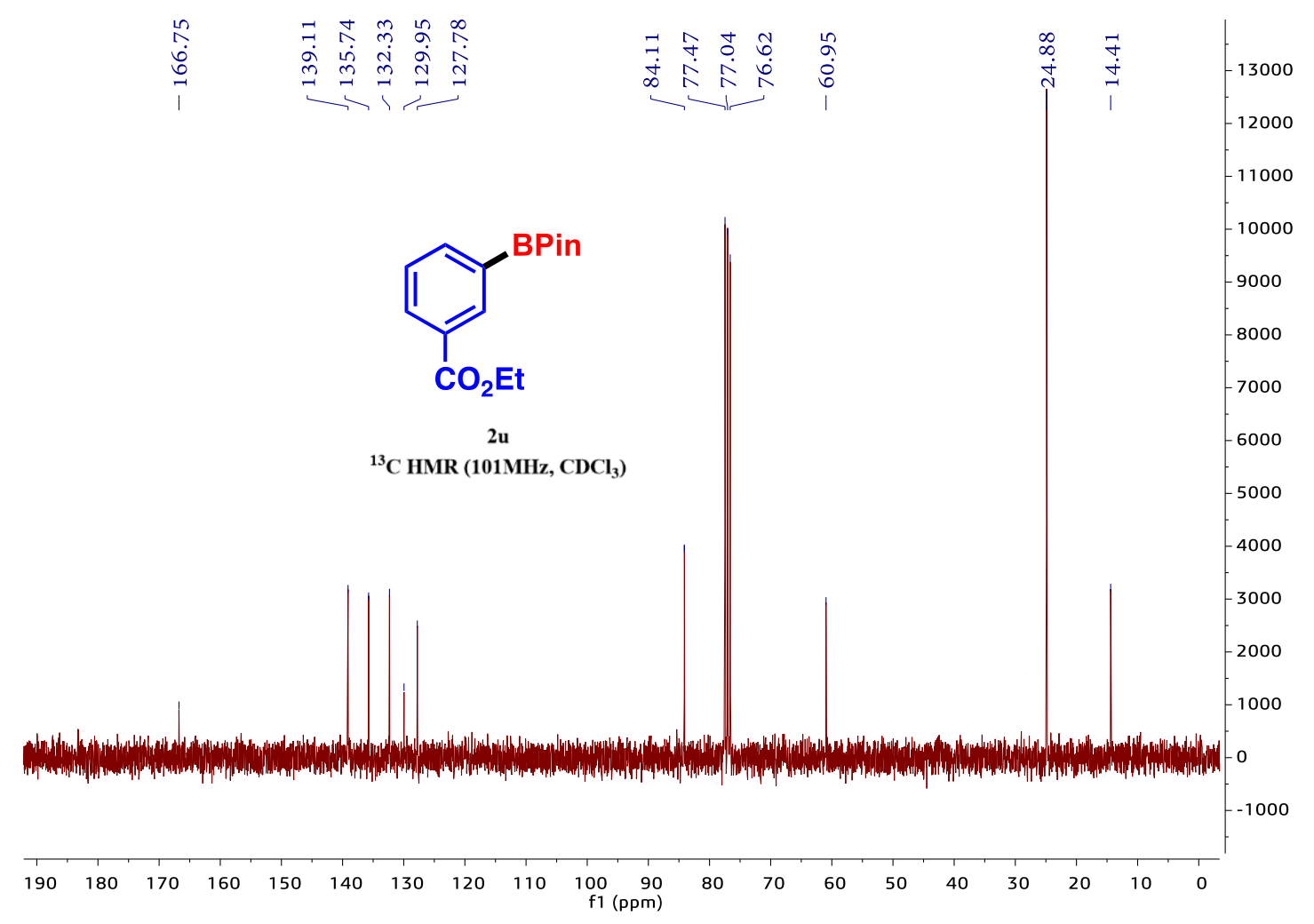

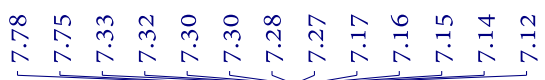

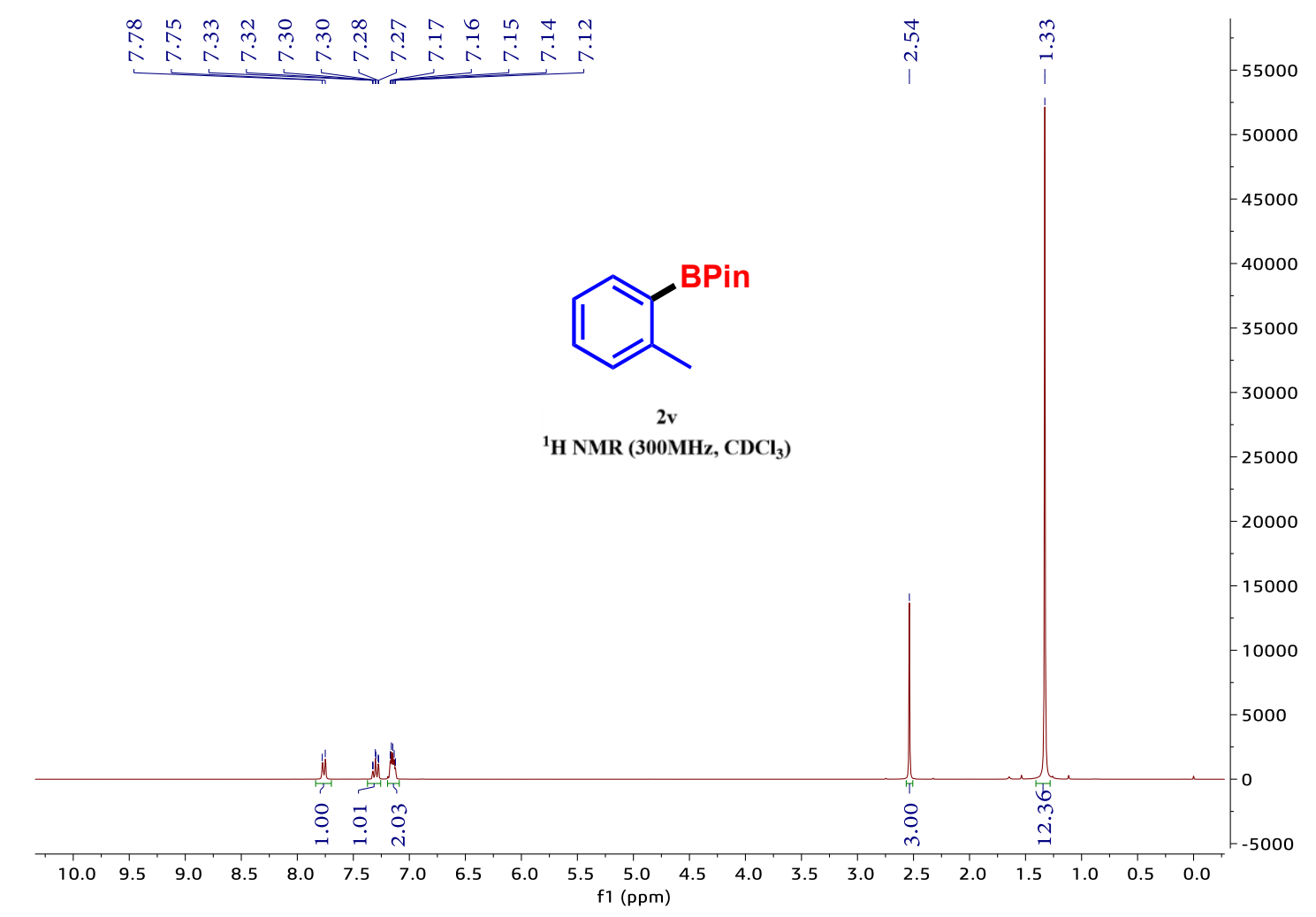



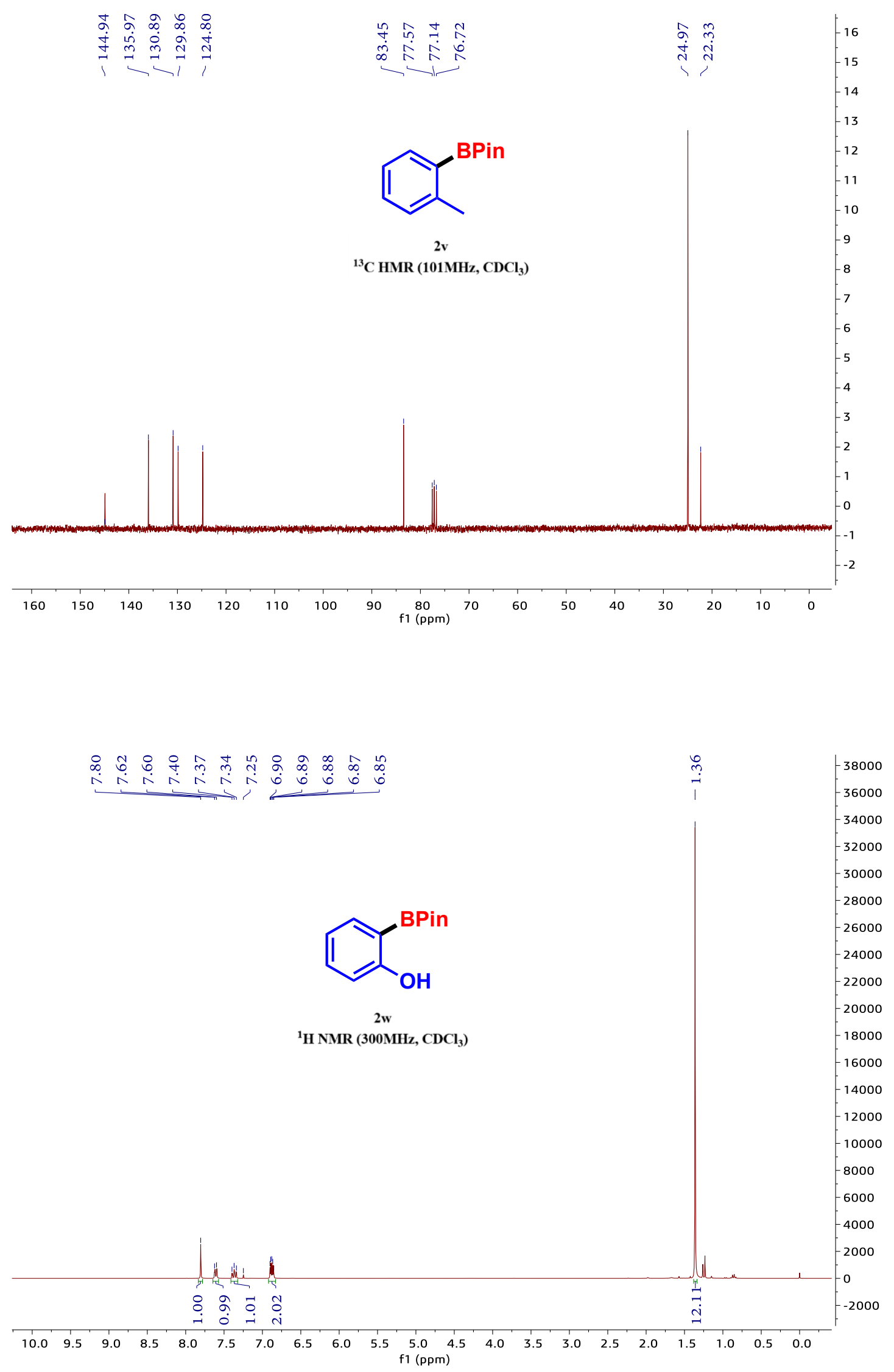

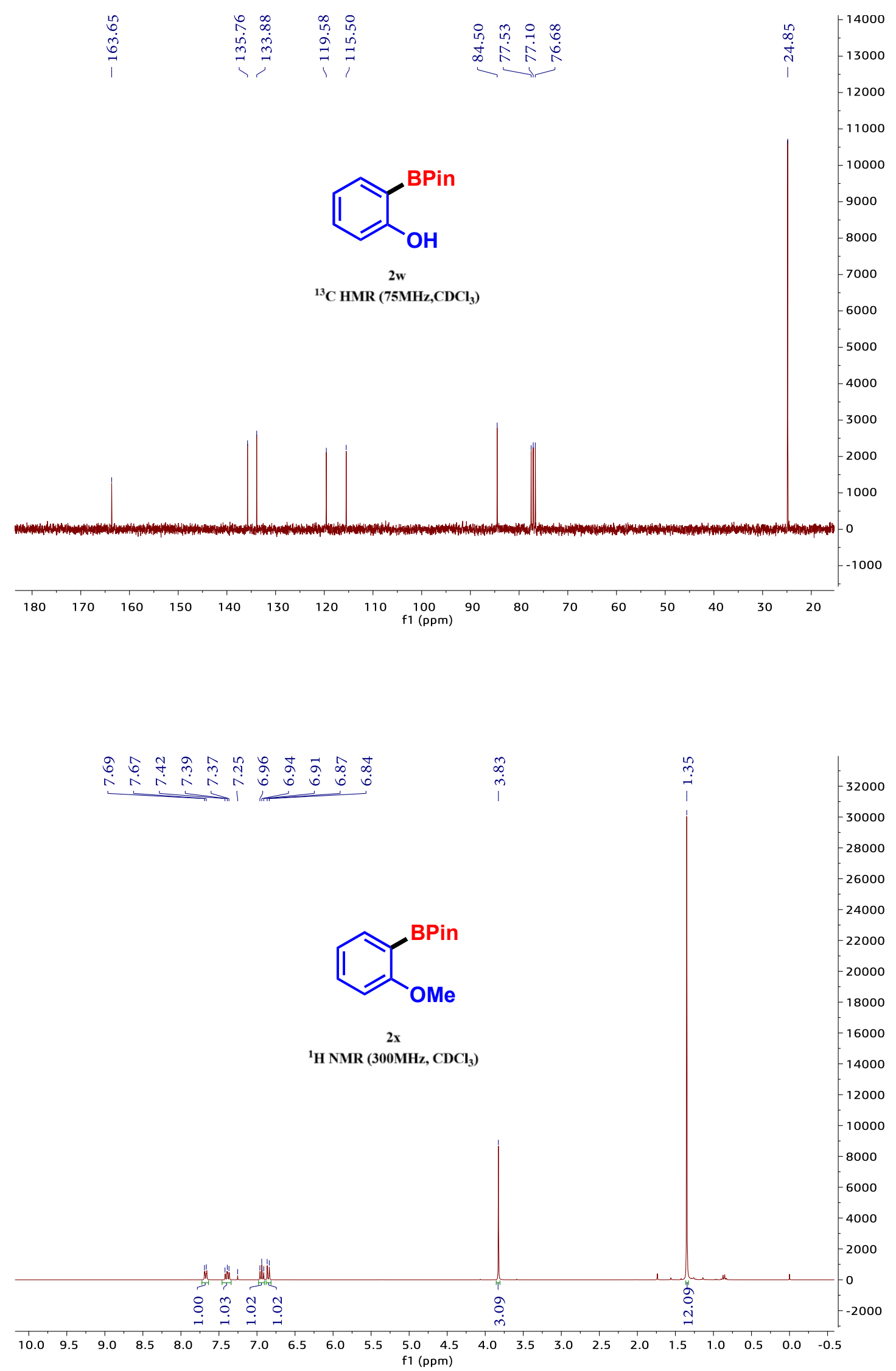


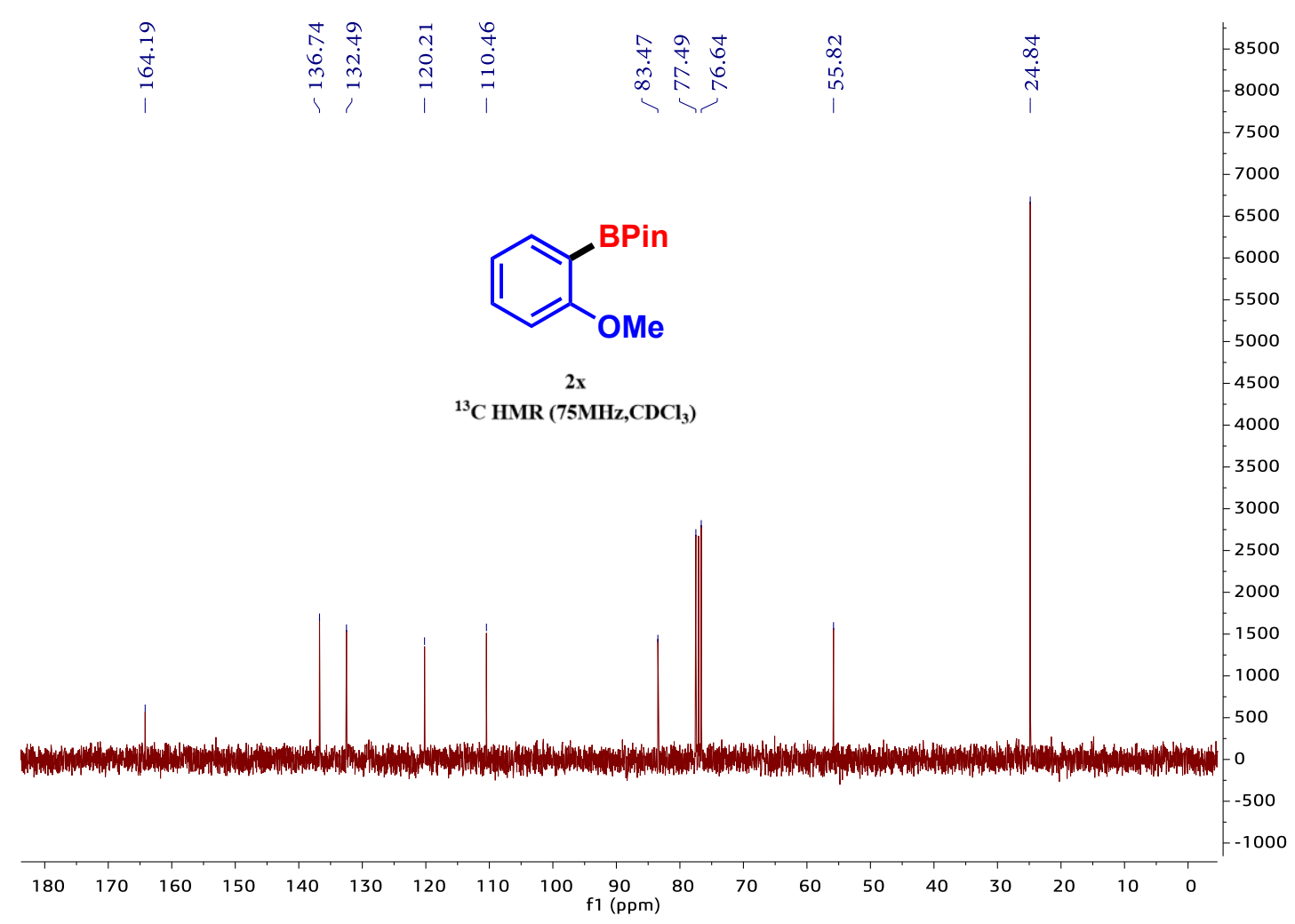

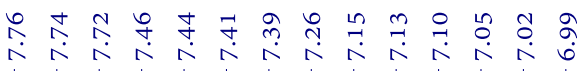

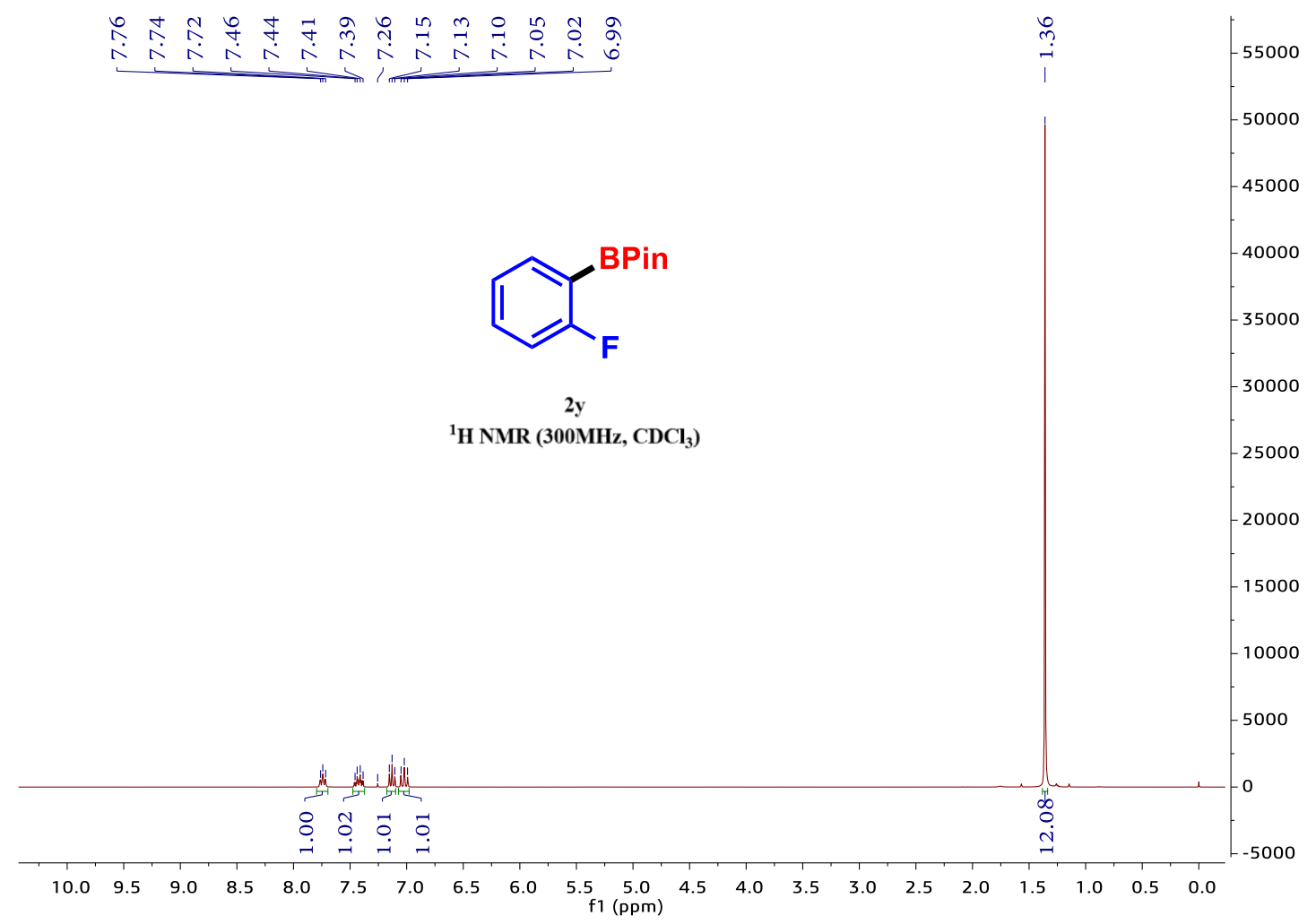




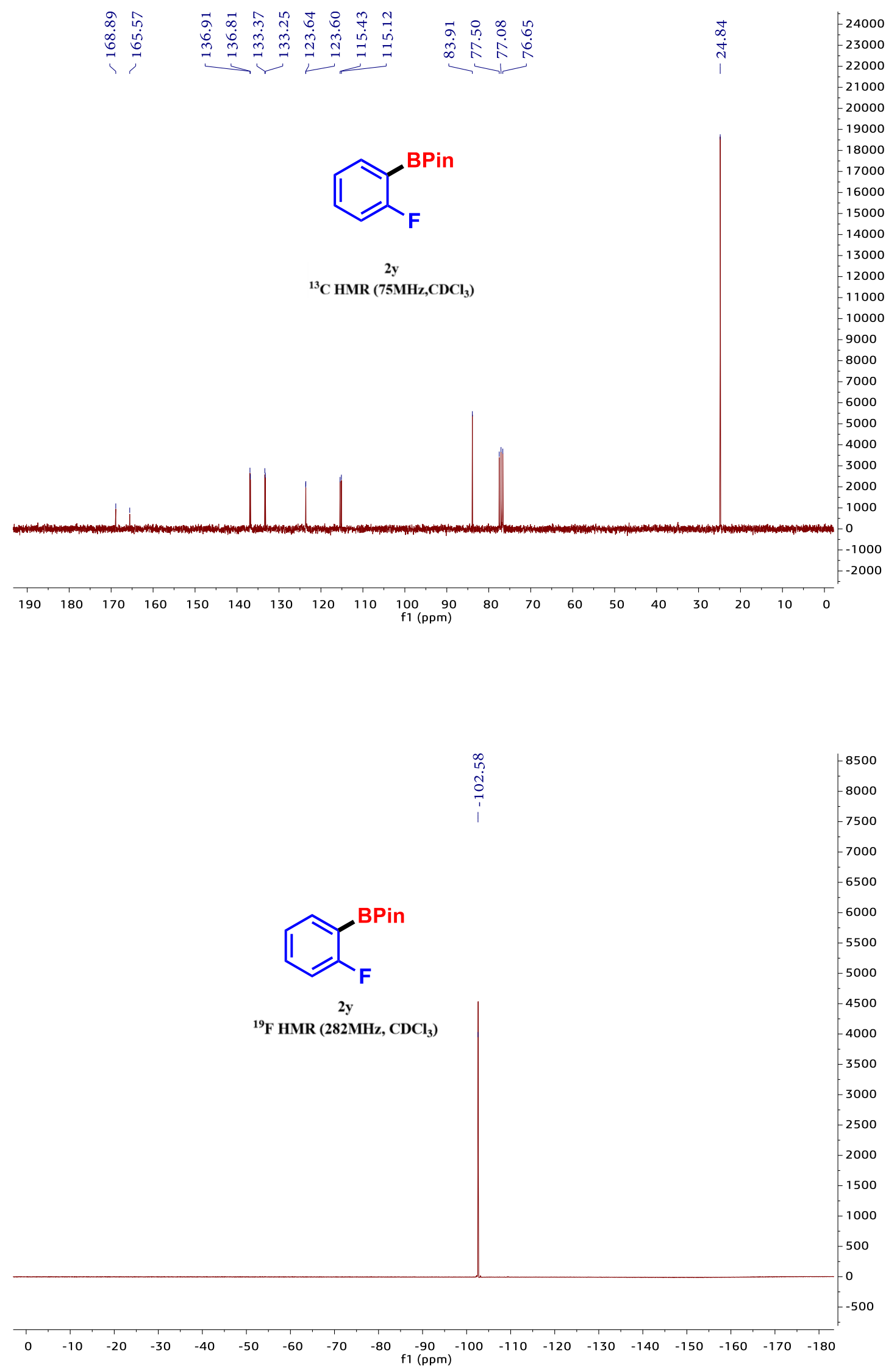



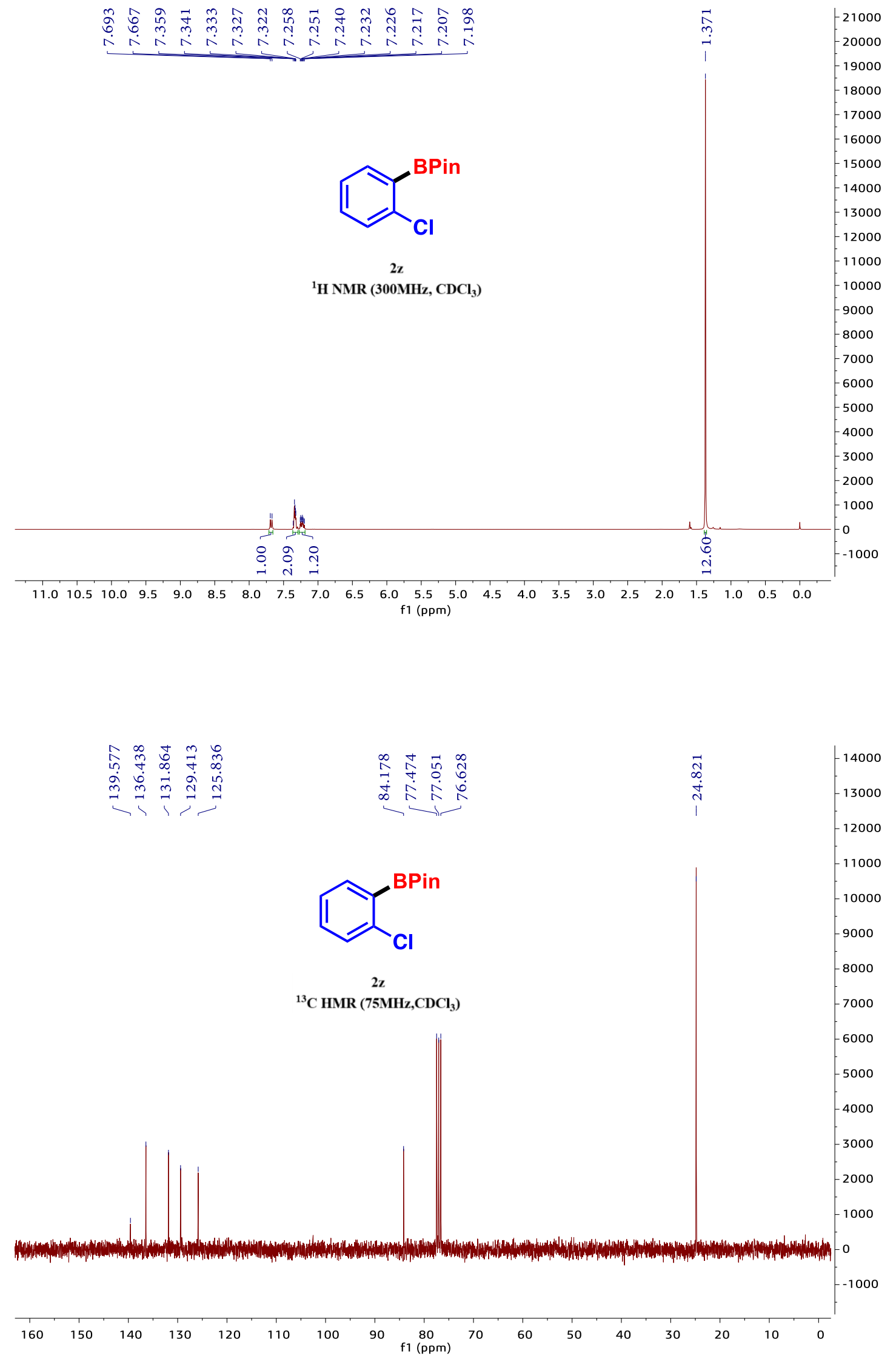

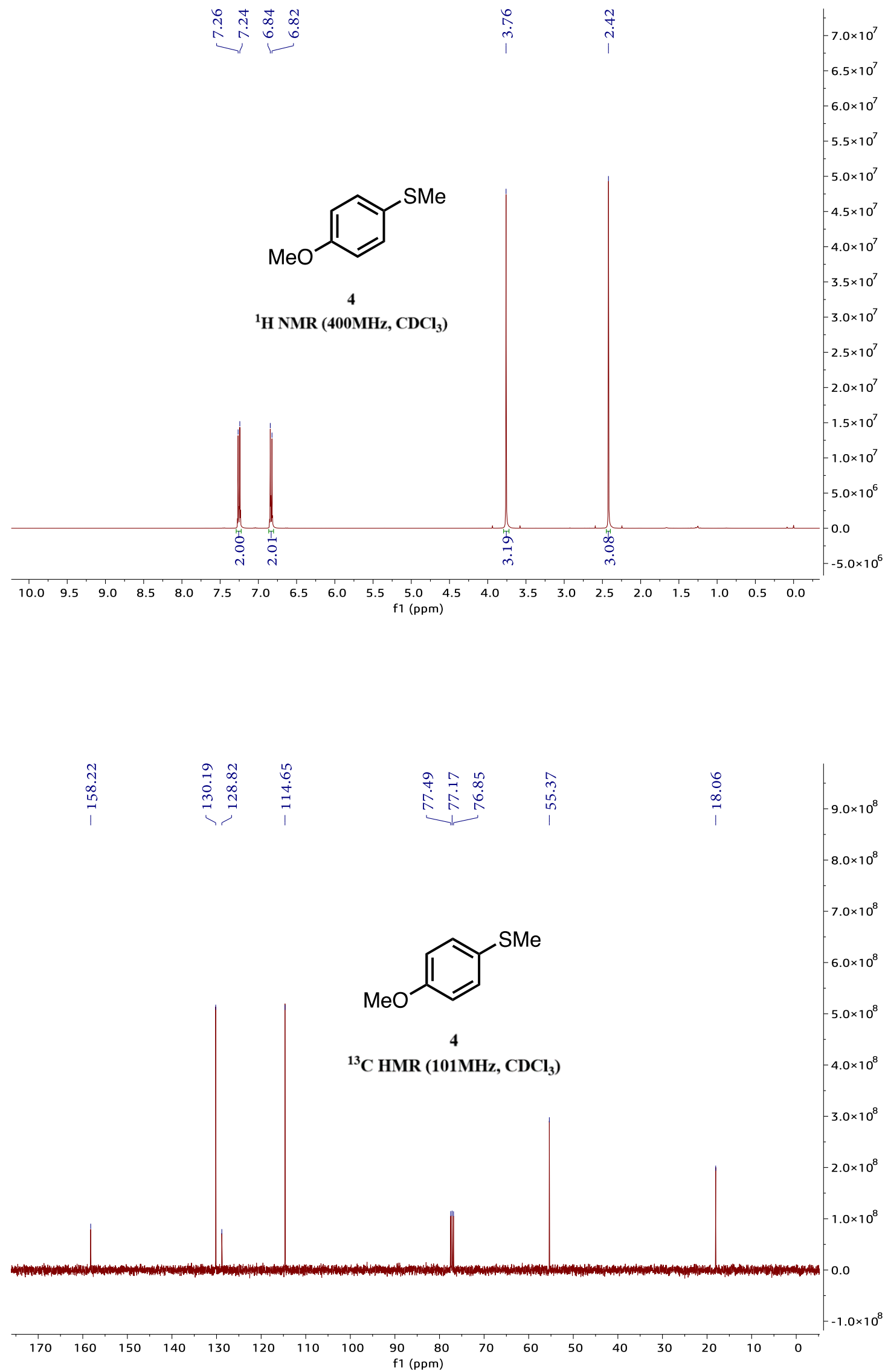